\title{
A higher-level phylogenetic classification of the Fungi
}

David S. HIBBETT ${ }^{a, *}$, Manfred BINDER ${ }^{a}$, Joseph F. BISCHOFF ${ }^{b}$, Meredith BLACKWELL ${ }^{c}$, Paul F. CANNON ${ }^{d}$, Ove E. ERIKSSON ${ }^{e}$, Sabine HUHNDORF ${ }^{f}$, Timothy JAMES ${ }^{g}$, Paul M. KIRK ${ }^{d}$, Robert LÜCKING ${ }^{f}, \mathrm{H}$. THORSTEN LUMBSCH ${ }^{f}$, François LUTZONI ${ }^{g}$, P. Brandon MATHENY $^{a}$, David J. MCLAUGHLIN ${ }^{h}$, Martha J. POWELL ${ }^{i}$, Scott REDHEAD ${ }^{j}$, Conrad L. SCHOCH ${ }^{k}$, Joseph W. SPATAFORA ${ }^{k}$, Joost A. STALPERS ${ }^{\text {, Rytas VILGALYS }}{ }^{g}$, M. Catherine AIME ${ }^{m}$, André APTROOT ${ }^{n}$, Robert BAUER ${ }^{\circ}$, Dominik BEGEROW ${ }^{p}$, Gerald L. BENNY ${ }^{q}$, Lisa A. CASTLEBURY ${ }^{m}$, Pedro W. CROUS ${ }^{l}$, Yu-Cheng DAI ${ }^{r}$, Walter GAMS ${ }^{l}$, David M. GEISER ${ }^{s}$, Gareth W. GRIFFITH ${ }^{t}$, Cécile GUEIDAN ${ }^{g}$, David L. HAWKSWORTH ${ }^{u}$, Geir HESTMARK ${ }^{v}$, Kentaro HOSAKA ${ }^{w}$, Richard A. HUMBER ${ }^{x}$, Kevin D. HYDE ${ }^{y}$, Joseph E. IRONSIDE ${ }^{t}$, Urmas KÕLJALG ${ }^{z}$, Cletus P. KURTZMAN ${ }^{a a}$, Karl-Henrik LARSSON ${ }^{a b}$, Robert LICHTWARDT ${ }^{a c}$, Joyce LONGCORE $^{\text {ad }}$, Jolanta MIACLIKOWSKA ${ }^{g}$, Andrew MILLER ${ }^{a e}$, Jean-Marc MONCALVO ${ }^{\text {af }}$, Sharon MOZLEY-STANDRIDGE ${ }^{a g}$, Franz OBERWINKLER ${ }^{\circ}$, Erast PARMASTO ${ }^{a h}$, Valérie REEB $^{g}$, Jack D. ROGERS $^{a i}$, Claude ROUX ${ }^{a j}$, Leif RYVARDEN ${ }^{a k}$, José Paulo SAMPAIO ${ }^{a l}$, Arthur SCHÜßLER $^{a m}$, Junta SUGIYAMA ${ }^{a n}$, R. Greg THORN $^{a o}$, Leif TIBELL ${ }^{a p}$, Wendy A. UNTEREINER $^{a q}$, Christopher WALKER ${ }^{a r}$, Zheng WANG ${ }^{a}$, Alex WEIR ${ }^{a s}$, Michael WEISS ${ }^{\circ}$, Merlin M. WHITE ${ }^{a t}$, Katarina WINKA ${ }^{e}$, Yi-Jian YAO ${ }^{a u}$, Ning ZHANG ${ }^{a v}$

\footnotetext{
aiology Department, Clark University, Worcester, MA 01610, USA

${ }^{b}$ National Library of Medicine, National Center for Biotechnology Information, 45 Center Drive, Bethesda, MD 20892-6510, USA

${ }^{\mathrm{C}}$ Department of Biological Sciences, Louisiana State University, Baton Rouge, LA 70803, USA

${ }^{d}$ CABI UK, Bakeham Lane, Egham, Surrey TW20 9TY, UK

${ }^{\mathrm{e}}$ Department of Ecology and Environmental Science, Umeå University, SE-901 87 Umeå, Sweden

fDepartment of Botany, The Field Museum of Natural History, 1400 South Lake Shore Drive, Chicago, IL 60605-2496, USA

'Department of Biology, Duke University, Box 90338, Durham, NC 27708, USA

h Department of Plant Biology, University of Minnesota, 1445 Gortner Avenue, St Paul, MN 55108-1095, USA

${ }^{i}$ Department of Biological Sciences, Box 870344/319 Biology, University of Alabama, Tuscaloosa, AL 35487-0344, USA

${ }_{\mathrm{j}}^{\mathrm{E} E C O R C}$, Agriculture and Agri-Food Canada, CEF, Neatby Building, Ottawa, ON K1A 0C6, Canada

${ }^{\mathrm{k}}$ Department of Botany and Plant Pathology, Oregon State University, 2082 Cordley Hall, Corvallis, OR 97331, USA

${ }^{1}$ Centraalbureau voor Schimmelcultures, Uppsalalaan 8, NL-3584 CT Utrecht, The Netherlands

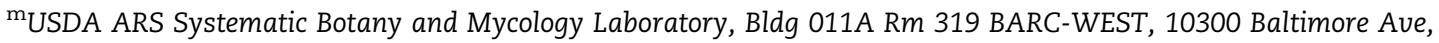

Beltsuille, MD 20705 USA

${ }^{\mathrm{n}}$ ABL Herbarium, Gerrit van der Veenstraat 107, NL-3762 XK Soest, The Netherlands

'Botanical Institute, University of Tübingen, Auf der Morgenstelle 1, D-72076 Tübingen, Germany

${ }^{\mathrm{p}}$ Max-Planck-Institute for Terrestrial Microbiology, Karl-von-Frisch-Strasse, D-35043 Marburg, Germany

${ }^{9}$ Department of Plant Pathology, 1453 Fifield Hall, Hull Road, P.O. Box 110680, University of Florida, Gainesville FL 32611-0680, USA

${ }^{\mathrm{r}}$ Institute of Applied Ecology, Chinese Academy of Sciences, Shenyang 110016, China

${ }^{s}$ Department of Plant Pathology, Pennsylvania State University, University Park, PA 16802, USA

tInstitute of Biological Sciences, University of Wales, Aberystwyth SY23 3DA, UK

uepartamento de Biología Vegetale II, Facultad de Farmacia, Universidad Complutense de Madrid, Plaza Ramón y Cajal, Ciudad Universitaria, E-28040 Madrid, Spain
}

\footnotetext{
* Corresponding author.

E-mail address: dhibbett@clarku.edu (D. S. Hibbett)

0953-7562/\$ - see front matter @ 2007 The British Mycological Society. Published by Elsevier Ltd. All rights reserved. doi:10.1016/j.mycres.2007.03.004
} 


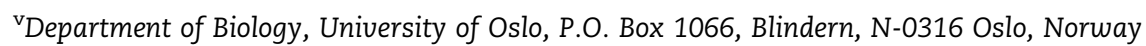

${ }^{w}$ Department of Botany, The Field Museum, 1400 S Lake Shore Dr., Chicago, IL 60605, USA,

${ }^{x}$ USDA-ARS Plant Protection Research Unit, US Plant, Soil and Nutrition Laboratory, Tower Road, Ithaca, NY 14853-2901, USA

${ }^{\mathrm{y}}$ Centre for Research in Fungal Diversity, Department of Ecology \& Biodiversity, University of Hong Kong, Pokfulam Road,

Hong Kong SAR, China

${ }^{\mathrm{z}}$ Institute of Botany and Ecology, Tartu University, 40 Lai Street, Tartu 51005, Estonia

${ }^{\text {aa }}$ Microbial Properties Research, NCAUR, ARS, USDA, 1815 N.University Street, Peoria, IL 61604-3999, USA

${ }^{\mathrm{ab}}$ Göteborg University, Department of Plant and Environmental Sciences, P. O. Box 461, SE-405 30 Göteborg, Sweden

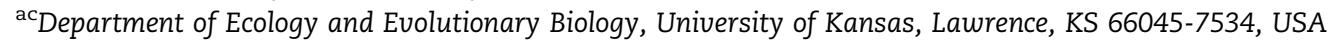

${ }^{\mathrm{ad}}$ Department of Biological Sciences, University of Maine, 5722 Deering Hall, Orono, ME 04469-5722, USA

${ }^{\text {ae }}$ Center for Biodiversity, Illinois Natural History Survey, 1816 South Oak Street, Champaign, IL 61820-6970, USA

${ }^{a f}$ Royal Ontario Museum, Centre for Biodiversity and Conservation Biology, 100 Queen's Park, Toronto, ON M5S 2C6, Canada

${ }^{a g}$ Division of Natural Sciences, Mathematics, and Engineering, Middle Georgia College, 1100 Second Street, SE, Cochran,

GA 31014-1599, USA

${ }^{\mathrm{ah}}$ Institute of Agricultural and Environmental Sciences, Estonian University of Life Sciences, 181 Riia Street Tartu, 51014, Estonia

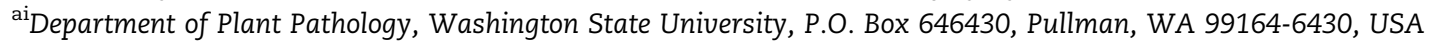

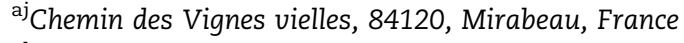

${ }^{a k}$ Botanical Institute, University of Oslo, P.O. Box 1066, Blindern, N-0316 Oslo, Norway

${ }^{a l}$ CREM, SABT, Faculdade de Ciências e Tecnologia, Universidade Nova de Lisboa, 2829-516 Caparica, Portugal

${ }^{a m}$ Genetics Institute, Department Biology I, Ludwig-Maximilians-University, Maria-Ward-Strasse 1a, D-80638 Munich, Germany

${ }^{a n}$ TechnoSuruga Co. Ltd, Tokyo Office, Ogawamachi Kita Building 4F, 1-8-3, Kanda Ogawamachi, Chiyoda-ku, Tokyo 101-0052, Japan

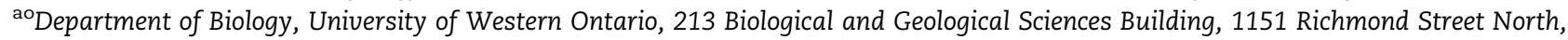
London, ON, N6A 5B7, Canada

${ }^{\mathrm{ap}}$ Department of Systematic Botany, Evolutionary Biology Centre, Uppsala University, Norbyuägen 18 D, SE-752 36, Uppsala, Sweden

${ }^{a q}$ Botany Department, Brandon University, 270-18th Street, Brandon, MB R7A 6A9, Canada

${ }^{\text {ar }}$ Royal Botanic Garden Edinburgh, 20A Inverlieth Row, Edinburgh Eh3 5LR, UK

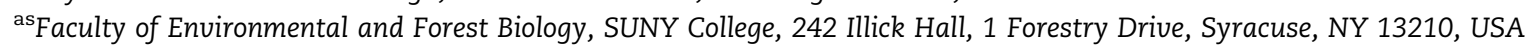

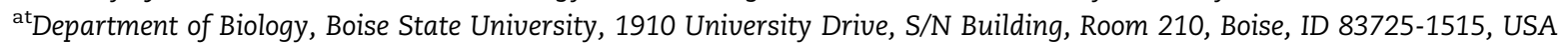

${ }^{\mathrm{au}}$ Systematic Mycology and Lichenology Laboratory, Institute of Microbiology, Chinese Academy of Sciences, Beijing 100080, China

${ }^{a v}$ Department of Plant Pathology, Cornell University, Geneva, NY 14456, USA

\section{A R T I C L E I N F O}

Article history:

Received 31 January 2007

Received in revised form

22 February 2007

Accepted 2 March 2007

Published online 13 March 2007

Corresponding Editor:

David L. Hawksworth

Keywords:

AFTOL

Eumycota

Lichens

Molecular phylogenetics

Mycota

Nomenclature

Systematics

\begin{abstract}
A B S T R A C T
A comprehensive phylogenetic classification of the kingdom Fungi is proposed, with reference to recent molecular phylogenetic analyses, and with input from diverse members of the fungal taxonomic community. The classification includes 195 taxa, down to the level of order, of which 16 are described or validated here: Dikarya subkingdom nov.; Chytridiomycota, Neocallimastigomycota phyla nov.; Monoblepharidomycetes, Neocallimastigomycetes class. nov.; Eurotiomycetidae, Lecanoromycetidae, Mycocaliciomycetidae subclass. nov.; Acarosporales, Corticiales, Baeomycetales, Candelariales, Gloeophyllales, Melanosporales, Trechisporales, Umbilicariales ords. nov. The clade containing Ascomycota and Basidiomycota is classified as subkingdom Dikarya, reflecting the putative synapomorphy of dikaryotic hyphae. The most dramatic shifts in the classification relative to previous works concern the groups that have traditionally been included in the Chytridiomycota and Zygomycota. The Chytridiomycota is retained in a restricted sense, with Blastocladiomycota and Neocallimastigomycota representing segregate phyla of flagellated Fungi. Taxa traditionally placed in Zygomycota are distributed among Glomeromycota and several subphyla incertae sedis, including Mucoromycotina, Entomophthoromycotina, Kickxellomycotina, and Zoopagomycotina. Microsporidia are included in the Fungi, but no further subdivision of the group is proposed. Several genera of 'basal' Fungi of uncertain position are not placed in any higher taxa, including Basidiobolus, Caulochytrium, Olpidium, and Rozella.

( 2007 The British Mycological Society. Published by Elsevier Ltd. All rights reserved.
\end{abstract}

\section{Introduction}

The molecular revolution in fungal taxonomy commenced in the early 1990s, with analyses of PCR-amplified ribosomal
RNA genes (White et al. 1990). Today, fungal molecular systematics is a mature discipline in which multi-locus datasets, extensive taxon sampling, and rigorous analytical approaches are standard. To gain an overview of the current state of the 
science it is only necessary to survey the recent 'Deep Hypha' issue of Mycologia [2007 ('2006'); 98], which contains 21 phylogenetic studies, all of which employ multiple genes to some extent (in some cases, multiple rRNA genes) and that address broad relationships in every major group of Fungi (except Microsporidia). Another recent milestone is the kingdom-level study of James et al. (2006), which used a dataset of six genes (nu-SSU, -LSU, and 5.8S rRNA, rpb1, rpb2 and tef1) sampled in nearly 200 species from every major clade of Fungi (including Microsporidia).

As the broad outlines of fungal phylogeny have come into focus, there have been repeated attempts to summarize the state of knowledge and to restructure higher-level classifications. Two important works that have influenced fungal taxonomy in the 21st century are Ainsworth \& Bisby's Dictionary of the Fungi (9th edn: Kirk et al. 2001), which contains a comprehensive kingdom-wide classification down to the level of genus, and The Mycota VII (McLaughlin et al. 2001a, 2001b), an edited volume with chapters on all major groups of Fungi. These publications represented major advances toward a phylogenetic classification of Fungi, but they are already out of date. In the five years since the last edition of the Dictionary and the Mycota VII appeared, more than 360 articles with the keyword 'phylogen*' were published in Mycologia and Mycological Research alone, and approximately $80 \%$ of the more than 100000 fungal rRNA gene sequences now in GenBank were deposited (some by molecular ecologists). Recent publications that survey the entire fungal kingdom based on molecular phylogenies include the chapter by Taylor et al. (2004) in Assembling the Tree of Life (Cracraft \& Donoghue 2004), the 'New Higher Level Classification of Eukaryotes' (Adl et al. 2005), and the first large collaborative analysis of the Assembling the Fungal Tree of Life (AFTOL) project (Lutzoni et al. 2004). Taxonomic studies on individual groups of Fungi are too numerous to list. Two notable highlights include proposals to recognize the phylum Glomeromycota (Schüßler et al. 2001) and to include the Microsporidia within the Fungi (Keeling et al. 2000).

On-line fungal taxonomies are also proliferating. One of the most important on-line general classifications of Fungi is that of GenBank (www.ncbi.nlm.nih.gov/Taxonomy), which serves a diverse community of researchers, including ecologists and molecular biologists. Another highly visible on-line classification is that of the Tree of Life Web Project (tolweb. org/tree), which is widely used by teachers and students. The classification of Ascomycota is being updated regularly via the on-line Myconet series (www.fieldmuseum.org/myconet), and this has been the basis for recent revisions at GenBank, but there is no comparable on-line resource for other major groups of Fungi. It is likely that on-line taxonomies will take on even greater prominence in the future, especially as they become integrated with databases of taxonomic names, particularly Index Fungorum (www.indexfungorum. org), MycoBank (www.mycobank.org), and other global biodiversity informatics resources (e.g. Global Biodiversity Information Facility, www.gbif.org).

Although there is broad agreement regarding the composition of the major clades of Fungi, there is considerable variation in the names that have been applied to these groups. For example, the clade that is called Basidiomycetes in the latest edition of the Dictionary is called Hymenomycetes at GenBank.
Similarly, the clade that is called Ascomycetes in the Dictionary of the Fungi is called Pezizomycotina in Myconet. Such inconsistencies create confusion, especially for students and nonspecialists, and they hamper efforts to develop taxonomic databases.

There is consequently a pressing need for the fungal systematics community to adopt a consensus higher-level classification for the Fungi that is based on well-supported monophyletic groups, and which can be recommended for general use. This is an opportune moment to create such a classification. With the new multi-locus analyses, many nodes that were not previously resolved are now supported with confidence. The timing is also good because there are multiple projects in progress that seek to create or update broad classifications of the Fungi. In particular, a tenth edition of the Dictionary is in preparation, as is a fourth edition of an influential textbook of mycology (Alexopoulos et al. 1996). The classifications used by GenBank, the Tree of Life Web Project, and Myconet are being revised continuously. If the classifications employed by these and other major taxonomic resources could be unified, it would promote communication and awareness of fungal phylogeny, and provide a framework for future revisions at all taxonomic levels.

This article presents a higher-level classification for all groups of Fungi, with reference to recent molecular phylogenetic studies. The authors represent diverse fungal taxonomy projects, including Ainsworth \& Bisby's Dictionary of the Fungi (Cannon, Kirk, Stalpers), GenBank (Bischoff), Myconet (Eriksson, Lumbsch, Huhndorf), and Alexopoulos' mycology text (Blackwell, Spatafora). Many of the authors are contributors to the Fungi pages in the Tree of Life Web Project. Discussions leading to this classification began in 2004, under the auspices of the AFTOL project and the Deep Hypha Research Coordination Network (Blackwell et al. 2007), which were supported by the US National Science Foundation. Throughout the development of this classification, every effort has been made to work in a transparent, consultative manner. The first draft classification was presented at the 2005 Deep Hypha meeting (Tucson, AZ) and subsequently was distributed to a group of 100 fungal systematists for comment. The classification was revised based on comments received and was posted on the AFTOL classification project web site (www.clarku.edu/ faculty/dhibbett/AFTOL/AFTOL.htm). Additional modifications were made following the 2006 Deep Hypha meeting (Baton Rouge, LA). For example, the classification of the Pucciniomycotina was revised to reflect the classification of Bauer et al. (2006). The present paper represents a first attempt at a broad-based consensus classification of the Fungi. However, the first 20 authors have exercised editorial control and are therefore to be held accountable for errors.

\section{Structure and principles}

This classification is restricted to organisms that belong in the monophyletic kingdom Fungi, including sexual and asexual forms. It does not consider other organisms formerly included in the kingdom but which are now known not to belong there, even if still studied by mycologists, such as the oomycetes and slime moulds. 
The classification adopted here uses a Linnean hierarchy as modified by the International Code of Botanical Nomenclature (Code) (McNeill et al. 2006), and uses seven ranks, including: order (suffix: -ales), subclass (-mycetidae), class (-mycetes), subphylum (-mycotina), phylum (-mycota; except Microsporidia), subkingdom, and kingdom. The rankings of taxa reflect the preferences and past practices of various authors, as well as the need to keep the nested hierarchies of clades and Linnean categories parallel. Taxa placed at the same rank are not necessarily equivalent in age (except sister taxa), number of species, or degree of morphological divergence.

The classification is limited to taxa down to the level of order. In many orders, especially those representing larger groups, such as Agaricales, there is still not enough resolution or taxon sampling to structure a comprehensive family-level classification. The challenge of creating family-level classifications is made even more difficult by the Code (McNeill et al. 2006), which requires that names of taxa at the rank of family or lower follow the principle of priority (which does not apply to higher ranks). Ideally, construction of consensus classifications within many of the orders recognized here will involve the coordinated efforts of groups of taxonomic specialists. It is hoped that the present classification will facilitate those endeavors.

The taxa included here are all supported as monophyletic by at least one published phylogenetic analysis (not applicable to monotypic taxa), with the exception of the Lahmiales and Triblidiales (Pezizomycotina) and Asellariales (Kickxellomycotina), for which molecular data are not available. Support for the monophyly of each group is summarized in three tables, which list selected phylogenetic studies, the type of data that were analysed, the number of OTUs sampled, and BS frequencies and Bayesian PPs. No attempt has been made to cite all of the relevant studies for each group. The analyses chosen for inclusion in the tables are those that have the greatest numbers of loci or taxa, and that provide the strongest support for monophyly of the clades in question. To supplement the information in the tables, brief comments on synonyms, phylogenetic relationships, and composition are provided below for some taxa, along with bibliographic citations for all taxon names. However, it is beyond the scope of this article to discuss each taxon in detail. For additional literature on the phylogeny and taxonomy of individual taxa, readers should consult the studies listed in the tables and below, and the references therein.

The classification is also presented as a set of three tree diagrams. Taxa of uncertain position are listed as incertae sedis, and have been placed at the least inclusive level in the hierarchy where they can be assigned with confidence. There are several nodes resolved in the tree figures that are not reflected in the classification. These unnamed clades, for which there is strong to moderate support in recent studies, include the Dacrymycetes plus Agaricomycetes (Basidiomycota) (Matheny et al. 2006, 2007a), Saccharomycotina plus Pezizomycotina (Ascomycota) (James et al. 2006; Spatafora et al. 2007), and the inoperculate euascomycetes (Ascomycota) (e.g. Lumbsch et al. 2002). The inoperculate euascomycetes have been recognized as a superclass, the Leotiomyceta (Eriksson \& Winka 1997; Lumbsch et al. 2002), which is a rank that is not employed here, while the Dacrymycetes plus Agaricomycetes correspond to the subclass Hymenomycetidae of Swann \& Taylor (1995). The absence of these groups from the present classification should not be interpreted as a judgment on their monophyly. Rather, it reflects a desire to keep the classification simple, and to minimize the number of intercalary ranks (as per the directives of Art. 4.3 of the Code). Future revisions to this classification will have to consider how to incorporate additional deep nodes, including those that will be resolved with the application of genome-scale datasets (Galagan et al. 2005; Kuramae et al. 2006; Robbertse et al. 2006). One possibility is to employ an unranked category (with or without a uniform suffix) that could be inserted at any level in the taxonomic hierarchy (Hibbett \& Donoghue 1998). For example, an unranked classification was adopted in part by Adl et al. (2005).

\section{Overview of the classification}

The classification accepts one kingdom, one subkingdom, seven phyla, ten subphyla, 35 classes, 12 subclasses, and 129 orders. Taxa that are described or validated here include Chytridiomycota, Monoblepharidomycetes, Neocallimastigomycota, Neocallimastigomycetes, Dikarya, Acarosporales, Baeomycetales, Candelariales, Umbilicariales, Lecanoromycetidae, Eurotiomycetidae, Mycocaliciomycetidae, Melanosporales, Corticiales, Gloeophyllales, and Trechisporales. Thus, about $90 \%$ of the 195 taxon names employed in the present classification have been validly published previously. The clade containing the Ascomycota and Basidiomycota is classified as the subkingdom Dikarya (as used in James et al. 2006), reflecting the putative synapomorphy of dikaryotic hyphae (Tehler 1988). All of the other new names are based on automatically typified teleomorphic names. The classification of Ascomycota largely parallels that of the Myconet classification, including recent changes that will be adopted in the forthcoming 2007 'Outline of the Ascomycota'. In Basidiomycota, the clades formerly called Basidiomycetes, Urediniomycetes, and Ustilaginomycetes in the last edition of Ainsworth \& Bisby's Dictionary of the Fungi are called the Agaricomycotina, Pucciniomycotina, and Ustilaginomycotina, respectively, as in Bauer et al. (2006). This is done to minimize confusion between taxon names and informal terms (basidiomycetes is a commonly used informal term for all Basidiomycota) and to refer to the included genera Agaricus (including the cultivated button mushroom) and Puccinia (which includes barberry-wheat rust). Another significant change in the Basidiomycota classification is the inclusion of the Wallemiomycetes and Entorrhizomycetes as classes incertae sedis within the phylum, reflecting ambiguity about their higher-level placements (Matheny et al. 2007b).

The most dramatic changes in the classification concern the 'basal fungal lineages', which include the taxa that have traditionally been placed in the Zygomycota and Chytridiomycota. These groups have long been recognized to be polyphyletic, based on analyses of rRNA, tef1, and rpb1 (James et al. 2000; Nagahama et al. 1995; Tanabe et al. 2004, 2005). The recent multilocus analyses of James et al. (2006) and others now provide the sampling, resolution, and support necessary to structure new classifications of these early-diverging groups, although significant questions remain. The Chytridiomycota is retained in a highly restricted sense, including 
Chytridiomycetes and Monoblepharidomycetes. The Blastocladiales, a traditional member of the Chytridiomycota, is here treated as a phylum, the Blastocladiomycota, as in James et al. (2007). The Neocallimastigales, whose distinctiveness from other chytrids has long been recognized, is also elevated to phylum, based on both morphology and molecular phylogeny. The genera Caulochytrium, Olpidium, and Rozella, which have traditionally been placed in the Chytridiomycota, and Basidiobolus, which has been classified in the Zygomycota (Entomophthorales), are not included in any higher taxa in this classification, pending more definitive resolutions of their placements.

The phylum Zygomycota is not accepted in this classification, pending resolution of relationships among the clades that have traditionally been placed in the Zygomycota (see discussion under Mucoromycotina). The traditional Zygomycota are here distributed among the phylum Glomeromycota and four subphyla incertae sedis, including Mucoromycotina, Kickxellomycotina, Zoopagomycotina and Entomophthoromycotina. A clade containing the Glomeromycota and the Dikarya was resolved previously based on ribosomal RNA genes and was classified as the Symbiomycota (Tehler et al. 2003). That taxon is not included here, because there was not strong support for the clade in the analyses of James et al. (2006) or Liu et al. (2006). If the Symbiomycota is added to this classification, it will need to be assigned a rank between kingdom and subkingdom, or perhaps be classified as an unranked taxon.

Microsporidia, unicellular parasites of animals and protists with highly reduced mitochondria (Germot et al. 1997; Hirt et al. 1997; Peyretaillade et al. 1998), are included here as a phylum of the Fungi, based on analyses by Keeling et al. (2000), Gill \& Fast (2006), James et al. (2006), and Liu et al. (2006). The latter study concluded that Microsporidia are the sister group of the rest of the Fungi and should not be classified as true Fungi, but that topology does not conflict with the delimitation of the monophyletic Fungi as proposed here. The analysis of James et al. (2006) suggested that Rozella, which was not sampled by Liu et al. (2006), is the sister group of the Microsporidia. No subdivision of the Microsporidia is proposed, owing to a lack of well-sampled multilocus analyses of this group (but see Vossbrinck \& Debrunner-Vossbrinck 2005, for an analysis using SSU rRNA genes).

\section{Phylogenetic classification of Fungi}

Many of the citations and authorities in the list below were obtained from the Index Fungorum databases (www. indexfungorum.org). A brief list of exemplar genera, including the type for automatically typified names, is given for each order (for small orders, all included genera are listed). A number of the genera listed are used in a modern, restricted sense, and readers are urged to consult the primary literature cited below and in the tables for information about current generic concepts. Comprehensive lists of genera and families included in each order will be forthcoming in the Dictionary of the Fungi (10th edn; listing on-line at www.indexfungorum.org) and in the next revision of Myconet (for Ascomycota). Further information on the names of fungi (not only kingdom
Fungi) above the rank of order and their places of publication may be found in the preliminary catalogue compiled by David (2002).

In accordance with the practice in recent editions of the Code, all scientific names regardless of rank are placed in italic type here except in the first line of the treatment of each accepted taxon where they are given in bold Roman type to make them stand out. When these names are used by other mycologists in their own publications, we wish to encourage the practice of the use of italics as recommended in the Preface to the current Code (McNeill et al. 2006).

Kingdom: Fungi R. T. Moore, Bot. Mar. 23: 371 (1980). Synonym: Fungi T. L. Jahn \& F. F. Jahn, How to Know the Protozoa: 7 (1949), nomen invalidum.

(Table 1, Fig 1)

The concept of the Fungi as one of six kingdoms of life was introduced by Jahn \& Jahn (1949), and a five kingdom system was advanced by Whittaker (1959), but neither of these works included a Latin diagnosis and the name was therefore invalid under the Code until the required Latin was provided by Moore (1980). Although Moore did not make a specific reference to Jahn \& Jahn's book, he was well aware that the name was in widespread use in the rank of kingdom. Under the current Code, Jahn \& Jahn are not to be included in the author citation. However, a proposal to change this provision in the Code will be made at the next International Botanical Congress (D. L. Hawksworth, unpubl.). If it is approved, the correct citation would be Fungi T. L. Jahn \& F. F. Jahn ex R. T. Moore (this rule change would also affect the citations of Ascomycota and Basidiomycota).

Phylum: Chytridiomycota M. J. Powell, phylum nov.

MycoBank no.: MB 501278

Synonyms: Archemycota Caval.-Sm., Biol. Rev. 73: 246 (1998), pro parte.

Thallus monocentricus vel polycentricus vel filamentosus; propagatio asexualis zoosporis, flagello retrorsum inserto, kinetosomate et centriolo supervacaneo praeditis, 9 munimentis flagelli, et complexu "microbody-corpore lipideo" descriptis; propagatio sexualis meiosi post copulationem perfecta; apparatus Golgi e cisternis superimpositis constans; tegumentum nuclei mitosi procedente circum polos fenestratum.

Typus: Chytridium A. Braun 1851.

Thallus monocentric, polycentric, or filamentous; asexual reproduction by zoospores with a single posteriorly-directed flagellum, both a kinetosome and non-functional centriole, nine flagellar props, and a microbody-lipid globule complex; sexual reproduction with zygotic meiosis where known; Golgi apparatus with stacked cisternae; nuclear envelope fenestrated at poles during mitosis.

Used as a phylum name without Latin diagnosis or description among others by von Arx (1967) and Margulis et al. (1990). Equivalent to euchytrids of James et al. 2006, the 'core chytrid clade' of James et al. (2007), or the 'core chytrid clade' plus the Monoblepharidales of James et al. (2000). Earlier usages are not indicated in the author citation of the name, because the circumscription adopted here differs significantly from that of those authors. 
Table 1 - Support for major groups of Fungi in selected phylogenetic studies: basal fungi and Dikarya

\begin{tabular}{|c|c|c|c|c|c|}
\hline Rank & Taxon & Reference & Data $^{a}$ & OTUs $^{b}$ & Support ${ }^{\mathrm{C}}$ \\
\hline \multirow[t]{4}{*}{ Kingdom } & FUNGI & Keeling (2003) & $\alpha$-tub, $\beta$-tub & 38 & MLBS $=98$ \\
\hline & & & & & NJBS $=94$ \\
\hline & & Baldauf et al. (2000) & act, $\alpha$-tub, $\beta$-tub, tef1 & 12 & MLBS $=85$ \\
\hline & & & & & MPBS $=95$ \\
\hline \multirow[t]{3}{*}{ Phylum } & CHYTRIDIOMYCOTA & James et al. (2007) & LSU, SSU, 5.8S & 84 & $\mathrm{BPP} \geq 0.95$ \\
\hline & & Seif et al. (2005) & mt-genome & 5 & $\mathrm{BPP}=1$ \\
\hline & & & & & MLBS $=100$ \\
\hline \multirow[t]{6}{*}{ Class } & Chytridiomycetes & James et al. (2006) & LSU, SSU, 5.8S, rpb1, rpb2, tef1 & 8 & $\mathrm{BPP} \geq 0.95$ \\
\hline & & & & & MLBS $\geq 70$ \\
\hline & & James et al. (2007) & LSU, SSU, 5.8S & 75 & $\mathrm{BPP} \geq 0.95$ \\
\hline & & & & & MLBS $\geq 70$ \\
\hline & & Keeling (2003) & $\alpha$-tub, $\beta$-tub & 5 & MLBS $=90$ \\
\hline & & & & & NJBS $=95$ \\
\hline Order & Chytridiales & James et al. (unpublished) & LSU, SSU, 5.8S, rpb1, rpb2, tef1, atp6 & 9 & MLBS $=98$ \\
\hline \multirow[t]{4}{*}{ Order } & Rhizophydiales & James et al. (2006) & LSU, SSU, 5.8S, rpb1, rpb2, tef1 & 2 & $\mathrm{BPP} \geq 0.95$ \\
\hline & & & & & MLBS $\geq 70$ \\
\hline & & Letcher et al. (2006) & LSU, $5.8 \mathrm{~S}$ & 96 & MPBS $=100$ \\
\hline & & & & & $\mathrm{BPP}=1$ \\
\hline Order & Spizellomycetales & James et al. (2007) & LSU, SSU, $5.8 \mathrm{~S}$ & 9 & MPBS $=100$ \\
\hline \multirow[t]{4}{*}{ Class/Order } & Monoblepharidomycetes, & James et al. (2007) & LSU, SSU, $5.8 \mathrm{~S}$ & 9 & $\mathrm{BPP} \geq 0.95$ \\
\hline & Monoblepharidales & & & & MLBS $\geq 70$ \\
\hline & & & & & MPBS $\geq 70$ \\
\hline & & Bullerwell et al. (2003) & $\operatorname{cox} 1,2,3 ; \operatorname{cob}$, atp6,9; nad 1,2,3,4, 4L,6 & 4 & MLBS $=100$ \\
\hline \multirow[t]{3}{*}{ Phylum/Class/Order } & NEOCALLIMASTIGOMYCOTA, & James et al. (2007) & LSU, SSU, $5.8 \mathrm{~S}$ & 6 & $\mathrm{BPP} \geq 0.95$ \\
\hline & Neocallimastigomycetes, & & & & MLBS $\geq 70$ \\
\hline & Neocallimastigales & & & & MPBS $\geq 70$ \\
\hline \multirow[t]{5}{*}{ Phylum/Class/Order } & BLASTOCLADIOMYCOTA, & James et al. (2007) & LSU, SSU, $5.8 \mathrm{~S}$ & 10 & $\mathrm{BPP} \geq 0.95$ \\
\hline & Blastocladiomycetes, & & & & \\
\hline & Blastocladiales & & & & \\
\hline & & Liu et al. (2006) & rpb1, rpb2 & 3 & $\mathrm{BPP}=1$ \\
\hline & & & & & MPBS $=100$ \\
\hline \multirow[t]{4}{*}{ Phylum } & MICROSPORIDIA & James et al. (2006) & LSU, SSU, 5.8S, rpb1, rpb2, tef1 & 2 & $\mathrm{BPP} \geq 0.95$ \\
\hline & & & & & MLBS $\geq 70$ \\
\hline & & Keeling (2003) & $\alpha$-tub, $\beta$-tub & 6 & MLBS $=100$ \\
\hline & & & & & NJBS $=97$ \\
\hline \multirow[t]{3}{*}{ Phylum/Class } & GLOMEROMYCOTA, & James et al. (2006) & LSU, SSU, 5.8S, rpb1, rpb2, tef1 & 5 & $\mathrm{BPP} \geq 0.95$ \\
\hline & Glomeromycetes & & & & MLBS $\geq 70$ \\
\hline & & Schüßler et al. (2001) & SSU & 72 & NJBS $\geq 90$ \\
\hline Order & Archaeosporales & Schüßler et al. (2001) & SSU & 5 & NJBS $\geq 95$ \\
\hline Order & Diversisporales & Schüßler et al. (2001) & SSU & 32 & NJBS $\geq 95$ \\
\hline Order & Glomerales & Schüßler et al. (2001) & SSU & 32 & NJBS $\geq 95$ \\
\hline \multirow[t]{2}{*}{ Order } & Paraglomerales & Schüßler et al. (2001) & SSU & 3 & $\mathrm{NJBS} \geq 95$ \\
\hline & $\begin{array}{l}\text { Subphyla incertae sedis } \\
\text { (not placed in any phylum) }\end{array}$ & & & & \\
\hline \multirow[t]{2}{*}{ Subphylum } & Mucoromycotina & James et al. (2006) & LSU, SSU, 5.8S, rpb1, rpb2, tef1 & 11 & $\mathrm{BPP}=1$ \\
\hline & & Tanabe et al. (2004) & rpb1 & 4 & $\mathrm{NJBS}=82$ \\
\hline \multirow[t]{7}{*}{ Order } & Mucorales & James et al. (2006) & LSU, SSU, 5.8S, rpb1, rpb2, tef1 & 3 & $\mathrm{BPP} \geq 0.95$ \\
\hline & & & & & MLBS $\geq 70$ \\
\hline & & Tanabe et al. (2004) & rpb1 & 3 & $\mathrm{NJBS}=100$ \\
\hline & & Keeling (2003) & $\alpha$-tub, $\beta$-tub & 4 & MLBS $=96$ \\
\hline & & & & & $\mathrm{NJBS}=98$ \\
\hline & & White et al. (2007) & LSU, SSU, 5.8S & 28 & $\mathrm{BPP}=1$ \\
\hline & & & & & MPBS $\geq 70$ \\
\hline \multirow[t]{2}{*}{ Order } & Endogonales & White et al. (2007) & LSU, SSU, $5.8 \mathrm{~S}$ & 2 & $\mathrm{BPP}=1$ \\
\hline & & & & & MPBS $\geq 70$ \\
\hline \multirow[t]{2}{*}{ Order } & Mortierellales & White et al. (2007) & LSU, SSU, $5.8 \mathrm{~S}$ & 6 & $\mathrm{BPP}=1$ \\
\hline & & & & & MPBS $\geq 70$ \\
\hline Subphylum/Order & Entomophthoromycotina, & James et al. (2006) & LSU, SSU, 5.8S, rpb1, rpb2, tef1 & 2 & $\mathrm{BPP} \geq 0.95$ \\
\hline & Entomophthorales & & & & MLBS $\geq 70$ \\
\hline Subphylum/Order & Zoopagomycotina, Zoopagales & James et al. (2006) & LSU, SSU, 5.8S, rpb1, rpb2, tef1 & 2 & $\mathrm{BPP} \geq 0.95$ \\
\hline & & & & & MLBS $\geq 70$ \\
\hline & & Tanabe et al. (2004) & $r p b 1$ & 3 & NJBS $=86$ \\
\hline Subphylum & Kickxellomycotina & Tanabe et al. (2004) & rpb1 & 6 & NJBS $=84$ \\
\hline Order & Kickxellales & O’Donnell et al. (1998) & SSU & 7 & MPBS $=100$ \\
\hline Order & Dimargaritales & Tanabe et al. (2000) & SSU & 3 & $\mathrm{NJBS}=100$ \\
\hline
\end{tabular}




\begin{tabular}{|c|c|c|c|c|c|}
\hline Rank & Taxon & Reference & Data $^{a}$ & OTUs $^{b}$ & Support ${ }^{\mathrm{C}}$ \\
\hline \multirow[t]{2}{*}{ Order } & Harpellales & Tanabe et al. (2004) & rpb1 & 3 & $\mathrm{NJBS}=98$ \\
\hline & & O'Donnell et al. (1998) & SSU & 4 & MPBS $=100$ \\
\hline Order & Asellariales & - & - & - & - \\
\hline \multirow[t]{4}{*}{ Subkingdom } & DIKARYA & James et al. (2006) & LSU, SSU, 5.8S, rpb1, rpb2, tef1 & 161 & $\begin{array}{l}\mathrm{BPP}=1 \\
\mathrm{MLBS}=71\end{array}$ \\
\hline & & Steenkamp et al. (2006) & act, $\alpha$-tub, $\beta$-tub, tef1 & 10 & $\begin{array}{l}\mathrm{BPP}=1 \\
\mathrm{MLBS}=84 \\
\mathrm{MPBS}=82 \\
\mathrm{NJBS}=96\end{array}$ \\
\hline & & Seif et al. (2005) & mt-genome & 10 & $\begin{array}{l}\mathrm{BPP}=1 \\
\mathrm{MLBS}=100\end{array}$ \\
\hline & & Liu et al. (2006) & $r p b 1, r p b 2$ & 27 & $\begin{array}{l}\mathrm{BPP}=1 \\
\mathrm{MPBS}=100\end{array}$ \\
\hline
\end{tabular}

Taxa with only one subsidiary taxon included (i.e. redundant taxa) are listed on a single line, with rank abbreviations divided by a slash (e.g. the class Agaricostilbomycetes, which contains a single order, Agaricostilbales, is indicated as Class/Order).

a LSU, SSU, and 5.8S refer to nuclear rRNA genes, whereas mt-LSU and mt-SSU refer to mitochondrial rRNA genes, other genes follow standard abbreviations. Some datasets contain missing sequences.

$\mathrm{b}$ Indicates the number of OTUs in the specified clade, not the total number of OTUs in the dataset.

c BS, bootstrap \%, jk, jackknife \%,WP = weighted parsimony, RML = RaxML, PML = PhyML, ME = minimum evolution, BPP, Bayesian posterior probability, NA, not applicable because the group is monotypic, or only a single species was sampled in the reference study.

Class: Chytridiomycetes Caval.-Sm., Biol. Rev. 73: 246 (1998).

Synonym: Archimycetes A. Fisch. (Fischer 1892) pro parte (included Olpidiopsis, Hypochytrium).

Type: Chytridium A. Braun 1851.

Reproducing asexually by zoospores bearing a single posteriorly-directed flagellum; zoospores containing a kinetosome

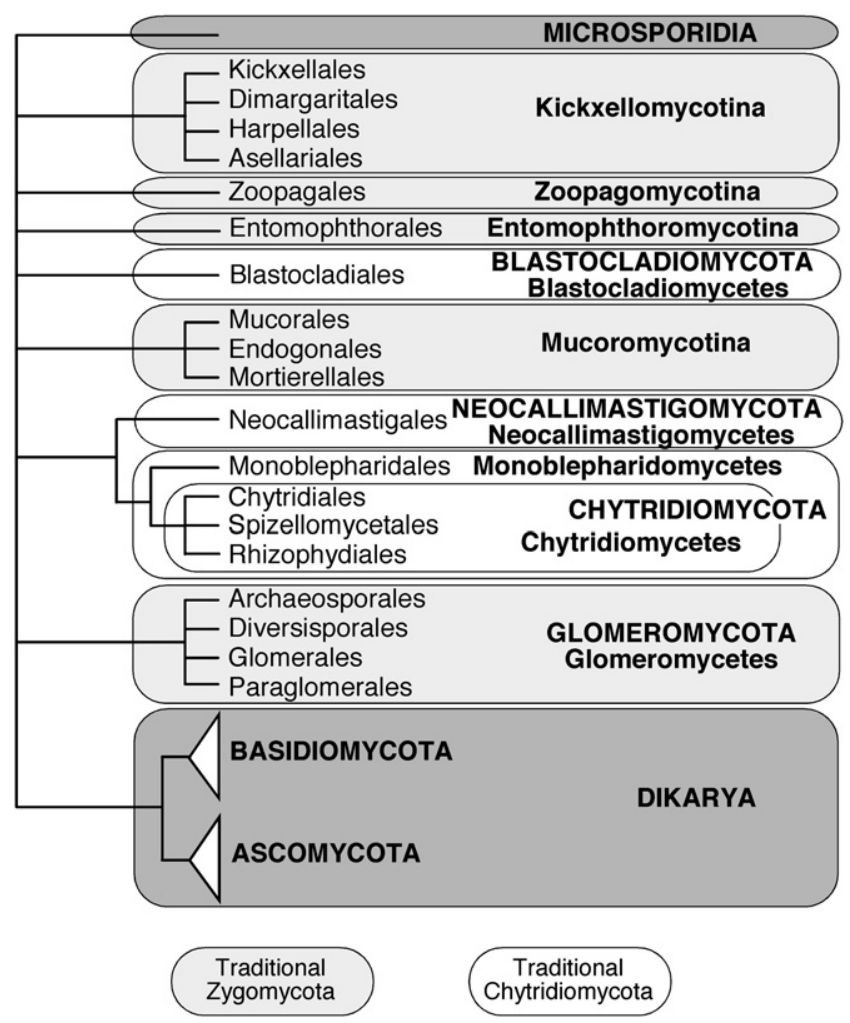

Fig 1 - Phylogeny and classification of Fungi. Basal Fungi and Dikarya. Branch lengths are not proportional to genetic distances. See Table 1 for support values for clades. and a non-flagellated centriole; thallus monocentric or rhizomycelial polycentric; sexual reproduction not oogamous.

Cavalier-Smith (1998) provided a brief, four-word, Latin description that was not diagnostic for phyla of uniflagellate fungi, and has been revised above. The name Chytridiomycetes was also used by Serbinow (1907), Cejp (1957), Sparrow (1958), and Alexopoulos et al. (1996). For further discussion of the nomenclatural history of the name, see David (2002).

Order: Chytridiales Cohn, Jber. schles. Ges. vaterl. Kultur 57: 279 (1879).

Emend. Schröter (as 'Chytridineae') in Engler \& Prantl, Nat. Pflanzenfam. 1: 64 (1892). Emend. Barr, Can. J. Bot. 58: 2384 (1980). Emend. Letcher \& Powell, Mycol. Res. 110: 907 (2006).

Type: Chytridium A. Braun 1851.

Thallus monocentric or polycentric rhizomycelial; zoospores typically with flagellar base containing an electron-opaque plug, microtubules extending from one side of the kinetosome in a parallel array, ribosomes aggregated near the nucleus, kinetosome parallel to non-flagellated centriole and connected to it by fibrous material, nucleus not associated with kinetosome, fenestrated cisterna (rumposome) adjacent to lipid globule.

Exemplar genera: Chytridium A. Braun 1851, Chytriomyces Karling 1945, Nowakowskiella J. Schröt. 1893.

An emmended description is presented above to conform to the circumscription adopted here. Monophyly of this group, as currently delimited, is not certain; Polychytrium Ajello 1942 and its allies and Chytriomyces angularis Longcore 1992 and its allies may eventually be segregated from Chytridiales s. str.

Order: Rhizophydiales Letcher, in Letcher et al., Mycol. Res. 110: 908 (2006).

Exemplar genera: Rhizophydium Schenk 1858, Kappamyces Letcher \& M.J. Powell 2005, Terramyces Letcher 2006, 
Boothiomyces Letcher 2006; Batrachochytrium Longcore, Pessier \& D.K. Nichols 1999 is on a long branch in this clade with no near relatives.

Order: Spizellomycetales D. J. S. Barr, Can. J. Bot. 58: 2384 (1980).

Exemplar genera: Spizellomyces D. J. S. Barr 1980, Powellomyces Longcore, D. J. S. Barr \& Désauln. 1995, Kochiomyces D. J. S. Barr 1980.

This classification does not include Caulochytrium, Olpidium, Rozella, or the Rhizophlyctis rosea clade, which are considered incertae sedis.

Class: Monoblepharidomycetes J. H. Schaffn., Ohio Nat. 9: 449 (1909), as 'Monoblepharideae'.

Type: Monoblepharis Cornu 1871.

Thallus filamentous, either extensive or a simple unbranched thallus, often with a basal holdfast; asexual reproduction by zoospores or autospores; zoospores containing a kinetosome parallel to a non-flagellated centriole, a striated disk partially extending around the kinetosome, microtubules radiating anteriorly from the striated disk, a ribosomal aggregation, and rumposome (fenestrated cisterna) adjacent to a microbody; sexual reproduction oogamous by means of posteriorly uniflagellate antherozoids borne in antheridia and nonflagellate female gametes borne in oogonia.

Schaffner (1909) used the name 'Monoblepharideae' as a class but with the ending of a suborder; this must be changed without change of authorship or date of publication (Code, Art. 16.3).

Order: Monoblepharidales J. Schröt., in Engler \& Prantl, Nat. Pflanzenfam. 1: 106 (1893), as 'Monoblepharidineae'.

Emend. Sparrow, Aquatic Phycomycetes: 458 (1943).

Emended description as for Monoblepharidomycetes.

Exemplar genera: Monoblepharis Cornu 1871, Harpochytrium Lagerh. 1890, Oedogoniomyces Tak. Kobay. \& M. Ôkubo 1954.

Phylum: Neocallimastigomycota M. J. Powell, phylum nov. MycoBank no.: MB 501279

Thallus monocentricus vel polycentricus; fungi anaerobici, intra tractum digestivum animalium herbivororum vel fortasse in substratis anaerobicis terrestribus vel limnicis; mitochondriis carentes sed hydrogenosomatibus praediti; zoosporae retrorsum uni- vel multiflagellatae, kinetosoma praesens sed centriolum supervacaneum absens; complexus kinetosomati affixus e radio marginali et annulo circumflagellari compositus; microtubuli e radio entendentes circum nucleum radiantes et flabellum posterius formantes; munimenta flagelli absentia; tegumentum nuclei mitosi procedente integrum remanens.

Typus: Neocallimastix Vavra \& Joyon ex I. B. Heath 1983.

Thallus monocentric or polycentric; anaerobic, found in digestive system of larger herbivorous mammals and possibly in other terrestrial and aquatic anaerobic environments; lacks mitochondria but contains hydrogenosomes of mitochondrial origin; zoospores posteriorly unflagellate or polyflagellate, kinetosome present but non-functional centriole absent, kinetosome-associated complex composed of a skirt, strut, spur and circumflagellar ring, microtubules extend from spur and radiate around nucleus, forming a posterior fan, flagellar props absent; nuclear envelope remains intact throughout mitosis.

Class: Neocallimastigomycetes M. J. Powell, class. nov. MycoBank no.: MB 501280

Diagnosis latina ut in Neocallimastigomycota (vide supra).

Typus: Neocallimastix Vavra \& Joyon ex I.B. Heath 1983.

Order: Neocallimastigales J. L. Li, I. B. Heath \& L. Packer, Can. J. Bot. 71: 403 (1993).

Exemplar genera: Neocallimastix Vavra \& Joyon ex I.B. Heath 1983, Caecomyces J.J. Gold 1988, Orpinomyces D.J.S. Barr, H. Kudo, Jakober \& K.J. Cheng 1989.

Phylum: Blastocladiomycota T. Y. James, Mycologia 98: 867 (2007) ['2006'].

Synonym: Allomycota Caval.-Sm., BioSystems 14: 465 (1981).

This phylum was proposed to reflect phylogenetic information from a number of molecular studies (James et al. 2007; Liu et al. 2006).

Class: Blastocladiomycetes T. Y. James, Mycologia 98: 868 (2007) ['2006'].

Synonym: Allomycetes Caval.-Sm., Biol. Rev. 73: 246 (1998), based on Allomyces E. J. Butler 1911.

Cavalier-Smith provided a brief, five-word Latin description for Allomycetes that is not diagnostic from other uniflagellate fungi. The name Allomycetes was not taken up, because it is appropriate to have a class name based on the same genus as an included ordinal name, and because Cavalier-Smith's 'diagnosis' was vague.

Order: Blastocladiales 1910, H. E. Petersen, Bot. Tidsskr. 29: 357 (1909) ('Blastocladiineae').

Exemplar genera: Allomyces E. J. Butler 1911, Blastocladia Reinsch 1877, Coelomomyces Keilin. 1921.

Phylum: Microsporidia Balbiani, C. R. Acad. Sci. Paris 95: 1168 (1882).

The nomenclatural status of Microsporidia is ambiguous. It has been treated as a phylum under the zoological Code (International Commission on Zoological Nomenclature 1999), but there is disagreement about the correct author citation (Larsson 2000; Sprague \& Becnel 1998), and it is uncertain if the name would be valid under the botanical Code. This uncertainty arises as Microsporidium Balbiani 1884 appears to be a later synonym of Nosema Naegeli 1857. The present work follows the recommendation of Sprague \& Becnel (1998) in attributing Microsporidia to Balbiani (1882), but this must be regarded as provisional. Before the status of the Microsporidia can be resolved, it will be necessary to decide whether the nomenclature of the group as a whole should be governed by the zoological or the botanical Code although the latter now allows names of fungi described under the zoological Code to be accepted. The final decision will require input from the community of scientists who study Microsporidia. 
No subdivision of the group is proposed here, owing to the lack of well-sampled multi-gene phylogenies within the group. However, Vossbrinck \& Debrunner-Vossbrinck (2005) proposed a class-level classification of microsporidia, based on small-subunit rRNA gene sequences.

Phylum: Glomeromycota C. Walker \& A. Schuessler, in Schüßler et al., Mycol. Res. 105: 1416 (2001).

Class: Glomeromycetes Caval.-Sm., Biol. Rev. 73: 246 (1998), as 'Glomomycetes'.

Synonym: Geomycetes Caval.-Sm., Biol. Rev. 73: 247 (1998).

Order: Archaeosporales C. Walker \& A. Schuessler, in Schüßler et al., Mycol. Res. 105: 1418 (2001).

Synonym: Geosiphonales Caval.-Sm., Biol. Rev. 73: 247 (1998).

Exemplar genera: Archaeospora J.B. Morton \& D. Redecker 2001, Geosiphon F. Wettst. 1915.

Order: Diversisporales C. Walker \& A. Schuessler, Mycol. Res. 108: 981 (2004).

Exemplar genera: Acaulospora Gerd. \& Trappe 1974, Diversispora C. Walker \& A. Schüßler 2004, Gigaspora Gerd. \& Trappe 1974, Pacispora Oehl \& Sieverd. 2004.

Order: Glomerales J. B. Morton \& Benny, Mycotaxon 37: 473 (1990), as 'Glomales'.

Exemplar genus: Glomus Tul. \& C. Tul. 1845.

Order: Paraglomerales C. Walker \& A. Schuessler, in Schüßler et al., Mycol. Res. 105: 1418 (2001).

Exemplar genus: Paraglomus J. B. Morton \& D. Redecker 2001.

\section{Subphyla incertae sedis (not assigned to any phylum):}

Subphylum: Mucoromycotina Benny, subphylum nov.

MycoBank no.: MB 501281

Fungi saprotrophici vel raro mycoparasiti facultativi, gallas facientes, haustoriis carentes, raro ectomycorrhizam facientes. Mycelium ramosum, juvene coenocyticum, maturum aliquando septis microporosis divisum. Reproductio asexualis sporangiis vel sporangiolis vel merosporangiis, raro chlamydosporis vel arthrosporis vel blastosporis effecta. Reproductio sexualis zygosporis plus minusve globosis e suspensoribus oppositis vel appositis formatis effecta.

Typus: Mucor Fresen. 1850.

Fungi saprobes, or rarely gall-forming, nonhaustorial, facultative mycoparasites, or forming ectomycorrhiza. Mycelium branched, coenocytic when young, sometimes producing septa that contain micropores at maturity. Asexual reproduction by sporangia, sporangiola, or merosporangia, or rarely by chlamydospores, arthrospores, or blastospores. Sexual reproduction by more or less globose zygospores formed on opposed or apposed suspensors.

This group includes the Mucorales, which is the core group of the traditional Zygomycota. Monophyly of the traditional Zygomycota (including Mucorales, Glomerales, Entomophthorales and Harpellales) was suggested by a recent study by Liu et al. (2006) using $r p b 1$ and $r p b 2$, but that finding conflicts with results of analyses that included additional loci and taxa, which suggested that the traditional Zygomycota is polyphyletic (James et al. 2006).

The name Zygomycota was first published without a Latin diagnosis by Moreau (1954) and is therefore invalid. At present, this classification does not include Zygomycota. When relationships among basal fungal lineages are more clearly resolved, it may be appropriate to resurrect and validate Zygomycota, to include Mucoromycotina and perhaps other clades.

Order: Mucorales Fr., Syst. Mycol. 3 (2): 296 (1832).

Exemplar genera: Mucor Fresen. 1850 ( pro parte), Parasitella Bainier 1903, Phycomyces Kunze 1823, Pilobolus Tode 1784, Rhizopus Ehrenb. 1821.

Order: Endogonales Moreau ex R. K. Benj., in Kendrick (ed.), Whole Fungus 2: 599 (1979).

Emend.: Morton \& Benny, Mycotaxon 37: 473 (1990).

Synonym: Endogonales Moreau, Encycl. Mycol. 23: 1231 (1954), nomen invalidum.

Exemplar genera: Endogone Link 1809, Peridiospora C. G. Wu \& S. J. Lin 1997, Sclerogone Warcup 1990, Youngiomyces Y. J. Yao 1995.

Order: Mortierellales Caval.-Sm., Biol. Rev. 73: 246 (1998).

Exemplar genera: Mortierella Coem. 1863, Dissophora Thaxt. 1914, Modicella Kanouse 1936.

Subphylum: Entomophthoromycotina Humber, subphylum nov.

MycoBank no.: MB 501282

Fungi pathogenici obligate animalibus (praecipue invertebratis) vel plantis cryptogamicis vel saprotrophici, interdum in animalibus vertebratis parasitici. Status somaticus mycelium coenocyticum vel septatum, pariete circumdatum vel protoplasticum, in hospite culturisve saepe corpora hyphalia multinucleata formans; forma protoplastica hyphoidea vel amoeboidea forma variabilis; cystidia et rhizoidea in aliquot speciebus athropodicolis formata. Characteres nuclei, sicut magnitudo, nucleoli magnitudo et locus, praesentia aut absentia heterochromatini intermitotici, familiis distinguendis iuvant. Conidiophora simplicia ramosave. Sporae primariae conidia vera, uninucleatae vel plurinucleatae vel multinucleatae, variis modis vi propulsae vel passive liberatae, conidia secundaria persaepe formata. Sporae perdurantes crassituncatae, bistratosae velut zygosporae post conjugationem velut azygosporae singulae formatae.

Typus: Entomophthora Fresen. 1856.

Obligate pathogens of animals (primarily arthropods), cryptogamic plants, or saprobes; occasionally facultative parasites of vertebrates. Somatic state consisting of a well-defined mycelium, coenocytic or septate, walled or protoplastic, which may fragment to form multinucleate hyphal bodies; protoplasts either hyphoid or amoeboid and changeable in shape; cystidia or rhizoids formed by some taxa. Such nuclear characters as overall size, location and comparative size of nucleoli, presence or absence of granular heterochromatin in chemically unfixed interphasic nuclei, and mitotic patterns are important at the family level. Conidiophores branched or unbranched. Primary spores true conidia, uni-, pluri-, or multinucleate, forcibly 
discharged by diverse possible means or passively dispersed; secondary conidia often produced. Resting spores with thick bi-layered walls form as zygospores after conjugations of undifferentiated gametangia from different or the same hyphal bodies or hypha or as azygospores arising without prior gametangial conjugations.

Order: Entomophthorales G. Winter, Rabenh. Krypt.-Fl. 1: 74 (1880).

Exemplar genera: Entomophthora Fresen. 1856, Ballocephala Drechsler 1951, Conidiobolus Bref. 1884, Entomophaga Batko 1964, Neozygites Witlaczil 1885.

Subphylum: Zoopagomycotina Benny, subphylum nov. MycoBank no.: MB 501283

Fungi endo- vel ectoparasitici microanimalium vel fungorum. Corpus vegetativum ex thallo simplici ramoso vel nonramoso vel mycelio nonseptato plus minusve extense ramoso constans. Ectoparasitae haustoria intra hospitem formantes. Reproductio asexualis arthrosporis, chlamydosporis vel sporangiolis uni- vel multisporis perfecta; sporangiosporae sporangiolorum multispororum in catenenis (merosporangiis) simplicibus vel ramosis dispositae. Reproductio sexualis zygosporis paene globosis perficitur; hyphae sexuales hyphis vegetativis similes vel plus minusve ampliatae.

Typus: Zoopage Drechsler 1935.

Endo- or ectoparasites of microanimals and fungi. Vegetative body consisting of a simple, branched or unbranched thallus or more of less extensively branched mycelium. Ectoparasites forming haustoria inside the host. Asexual reproduction by arthrospores, chlamydospores or uni- or multispored sporangiola; sporangiospores of multispored sporangiola formed in simple or branched chains (merosporangia). Sexual reproduction by nearly globose zygospores; sexual hyphae similar to the vegetative hyphae or more or less enlarged.

The description of this group is based mostly on the validating description for the Zoopagales by Benjamin (1979), except that arthrospores have been added, based on Barron's (1975) report of arthrospores in Helicocephalum Thaxt. 1891.

Order: Zoopagales Bessey ex R.K. Benj., in Kendrick (ed.), Whole Fungus 2: 590 (1979).

Synonym: Zoopagales Bessey, Morph. Tax. Fungi : 177 (1950), nomen invalidum.

Exemplar genera: Cochlonema Drechsler 1935, Rhopalomyces Corda 1839, Piptocephalis de Bary 1865, Sigmoideomyces Thaxt. 1891, Syncephalis Tiegh. \& G. Le Monn. 1873, Zoopage Drechsler 1935.

Subphylum: Kickxellomycotina Benny, subphylum nov. MycoBank no.: MB 501284

Fungi saprotrophici vel mycoparasitici vel obligate symbiotici. Thallus in nonnullis generibus e tenaculo fungos alios parasitans et haustoriis penetrans; mycelium septatum, ramosum vel simplex; septa in medio excavata et obturata. Reproductio asexualis merosporangiis uni- vel bisporis vel trichosporis vel arthrosporis effecta. Reproductio sexualis zygosporis globosis, biconicis vel allantoideis circinatis effecta.

Typus: Kickxella Coem. 1862.
Fungi saprobes, mycoparasites, or obligate symbionts. Thallus arising from a holdfast on other fungi as a haustorial parasite, or branched, septate, subaerial hyphae. Mycelium branched or unbranched, regularly septate. Septa with median, disciform cavities containing plugs. Asexual production by 1- or 2-spored merosporangia, trichospores, or arthrospores. Sexual reproduction by zygospores that are globose, biconical, or allantoid and coiled.

Order: Kickxellales Kreisel ex R. K. Benj., in Kendrick (ed.), Whole Fungus 2: 610 (1979).

Synonym: Kickxellales Kreisel, Grundz. nat. Syst. Pilze: 65 (1969), nomen invalidum.

Exemplar genera: Kickxella Coem. 1862, Coemansia Tiegh. \& G. Le Monn. 1873, Linderina Raper \& Fennell 1952, Spirodactylon R. K. Benj. 1959.

Order: Dimargaritales R. K. Benj., in Kendrick (ed.), Whole Fungus 2: 607 (1979).

Exemplar genera: Dimargaris Tiegh. 1875, Dispira Tiegh. 1875, Tieghemiomyces R. K. Benj. 1959.

Order: Harpellales Lichtw. \& Manier, Mycotaxon 7: 441 (1978).

The taxa in this order have been referred to as 'Trichomycetes'. However, Trichomycetes is no longer a useful phylogenetic taxon because it describes a polyphyletic group. The use of the term should be restricted to ecological rather than phylogenetic groupings, and not capitalized or italicized, i.e. as 'trichomycetes'.

Exemplar genera: Harpella L. Léger \& Duboscq 1929, Furculomyces Lichtw. \& M. C. Williams 1992, Legeriomyces Pouzar 1972, Smittium R. Poiss. 1937.

Order: Asellariales Manier ex Manier \& Lichtw., Mycotaxon 7: 442 (1978).

Exemplar genera: Asellaria R. Poiss. 1932, Orchesellaria Manier ex Manier \& Lichtw. 1968.

Asellariales are retained in the Fungi here due to their ultrastructural characteristics (Benny \& White 2001; Manier 1973; Moss 1975; Saikawa et al. 1997). Unpublished rpb1 and rpb2 data also support their placement in the Kickxellomycotina (T. Y. James \& M. M. White, unpubl.).

Subkingdom: Dikarya Hibbett, T. Y. James \& Vilgalys, subregnum nov.

MycoBank no.: MB 501285

Synonyms: Neomycota Caval.-Sm., Rev. Biol. 73: 209 (1998).

Carpomycetaceae Bessey, Univ. Studies, Univ. Nebr. 7: 294 (1907).

Fungi unicellulares vel filamentosi, flagellis carentes, saepe stadium dikaryoticum includentes. Ascomycota et Basidiomycota complectens.

Unicellular or filamentous Fungi, lacking flagella, often with a dikaryotic state. The least-inclusive clade that contains Ascomycota and Basidiomycota. 
The name alludes to the putative synapomorphy of dikaryotic hyphae (Tehler 1988) and was applied by James et al. (2006) without formal description. Kendrick (1985) and Tehler et al. (2003) referred to this group as the Dikaryomycota, but the termination '-mycota' denotes the rank of phylum under the Code. CavalierSmith (1998) referred to this group as Neomycota. Dikarya is used here, because it is more descriptive and is consistent with recent use (James et al. 2006; Tehler et al. 2003; Kendrick 1985).

Phylum: Ascomycota Caval.-Sm., Biol. Rev. 73: 247 (1998), as 'Ascomycota Berk. 1857. stat. nov.'

Synonyms: Ascomycetes Berk., Intr. Crypt. Bot.: 270 (1857), rank uncertain; Whittaker (1959: 220).

Ascomycota Bold, Morph. Pl.: 7, 180 (1958), nomen invalidum; Hawksworth et al. (1995: 30), Eriksson \& Winka (1997: 4), etc, nomina nuda.

Basic type: Peziza Fr. 1822.

(Table 2, Fig 2) Cavalier-Smith was not the first to propose the phylum name Ascomycota. It appears to have been used first by Bold (1957: 7, 180), but without a Latin diagnosis. The name was in widespread use before its validation by Cavalier-Smith, and its usage was popularized by its employment in the eighth edition of the Dictionary, which is listed in Cavalier-Smith's (1998) bibliography. The Latin diagnosis provided by CavalierSmith consisted of only two words: 'sporae intracellulares'. It is questionable whether this description is diagnostic for the Ascomycota, but as a validating diagnosis it is acceptable under the Code. No detailed reference to the basionym was given, but is provided here. We also propose a basic type, Peziza, as we can not be sure that the phylum will not be split in the future when more molecular data and material of ascomycetes and basidiomycetes have been sequenced. Hawksworth et al. (1995) and Eriksson \& Winka (1997: 4) used the phylum names Ascomycota and Basidiomycota; the latter authors listed 31 nucleotide signatures in the nSSU rDNA genes in Basidiomycota. Since then many more sequences have become available, also from many other genes that support monophyly of Ascomycota and Basidiomycota.

The subdivision of Ascomycota used in the present paper is based on the system of Eriksson \& Winka (1997), which differs in many respects from that of Cavalier-Smith (1998).

Subphylum: Taphrinomycotina O. E. Erikss. \& Winka, Myconet 1: 11 (1997).

Class: Taphrinomycetes O. E. Erikss. \& Winka, Myconet 1: 11 (1997).

Order: Taphrinales Gäum. \& C. W. Dodge, Comp. morph. fun.: 159 (1928). 1832.

Exemplar genera: Taphrina Fr. 1815, Protomyces Unger

Class: Neolectomycetes O. E. Erikss. \& Winka, Myconet 1: 8 (1997).

Order: Neolectales Landvik, O. E. Erikss, Gargas \& P. Gustafss., Syst. Ascom. 11: 114 (1993).
Exemplar genus: Neolecta Speg. 1881.

Class: Pneumocystidomycetes O. E. Erikss. \& Winka, Myconet 1: 9 (1997).

Order: Pneumocystidales O. E. Erikss., Syst. Ascom. 13: 170 (1994).

Exemplar genus: Pneumocystis P. Delanoë \& Delanoë 1912.

Class: Schizosaccharomycetes O. E. Erikss. \& Winka, Myconet 1: 10 (1997).

Order: Schizosaccharomycetales O. E. Erikss., Svedskog \& Landvik, Syst. Ascom. 11: 146 (1993).

Exemplar genus: Schizosaccharomyces Linder 1893.

Subphylum: Saccharomycotina O. E. Erikss. \& Winka, Myconet 1: 10 (1997).

Class: Saccharomycetes O. E. Erikss. \& Winka, Myconet 1: 10 (1997).

Order: Saccharomycetales Kudryavtsev, System Hefen: 270 (1960).

Growth usually by individual yeast cells, often accompanied by pseudohyphae and/or true hyphae. Cell walls predominately of $\beta$-glucan. Ascomata not formed; one to many ascospores formed in asci that often are converted from individual cells or borne on simple ascophores. Mitotic and meiotic nuclear divisions within an intact nuclear membrane. Enveloping membrane system in ascospore delimitation associated independently with postmeiotic nuclei. Asexual reproduction by holoblastic budding, conidia or fission (arthrospores).

Exemplar genera: Saccharomyces Meyen ex E. C. Hansen 1838, Candida Berkhout 1923, Dipodascopsis L. R. Batra \& Millner 1978, Metschnikowia T. Kamieński 1899.

Subphylum: Pezizomycotina O. E. Erikss. \& Winka, Myconet 1: 9 (1997).

Class: Arthoniomycetes O. E. Erikss. \& Winka, Myconet 1: 4 (1997).

Order: Arthoniales Henssen \& Jahns ex D. Hawksw. \& O. E. Erikss, Syst. Ascom. 5: 177 (1986).

Synonym: Arthoniales Henssen \& Jahns, Lichenes: 123 (1973) ['1974'], nomen invalidum.

Hawkworth \& Eriksson (loc. cit.) listed only Henssen, but cited the book by Henssen \& Jahns (loc. cit.) as place for the original but invalid description so both should be cited although Henssen contributed the taxonomic system to the book.

Exemplar genera: Arthonia Ach. 1806, Chrysothrix Mont. 1852, Dirina Fr. 1825, Roccella DC. 1805.

Class: Dothideomycetes O. E. Erikss. \& Winka, Myconet 1: 5 (1997). 
Table 2 - Support for major groups of Fungi in selected phylogenetic studies: Ascomycota

\begin{tabular}{|c|c|c|c|c|c|}
\hline Rank & Taxon & Reference & Data & OTUs & Support \\
\hline \multirow[t]{3}{*}{ Phylum } & ASCOMYCOTA & James et al. (2006, fig. 1) & $\begin{array}{l}\text { SSU, LSU, 5.8S, } \\
r p b 1, r p b 2, \text { tef1 }\end{array}$ & 111 & $\begin{array}{l}\mathrm{MLBS}=94 \\
\mathrm{BPP}=1\end{array}$ \\
\hline & & Spatafora et al. (2007, fig. 2) & $\begin{array}{l}\text { LSU, SSU, rpb1, } \\
r p b 2 \text {, tef1 }\end{array}$ & 177 & $\begin{array}{l}\text { WPBS }=<50 \\
\text { MLBS }=100 \\
\text { BPP }=1\end{array}$ \\
\hline & & Lutzoni et al. (2004, fig. 2) & LSU, SSU & 276 & $\begin{array}{l}\mathrm{NJBS}=67 \\
\mathrm{BPP}=1\end{array}$ \\
\hline \multirow[t]{5}{*}{ Subphylum } & Taphrinomycotina & James et al. (2006, fig. 2) & $\begin{array}{l}\text { SSU, LSU, 5.8S, } \\
r p b 1, r p b 2, \text { tef1 }\end{array}$ & 4 & $\begin{array}{l}\mathrm{MLBS}=98 \\
\mathrm{BPP}=1\end{array}$ \\
\hline & & Spatafora et al. (2007, fig. 2) & $\begin{array}{l}\text { LSU, SSU, rpb1, } \\
r p b 2 \text {, tef1 }\end{array}$ & 8 & $\begin{array}{l}\mathrm{WPBS}=<50 \\
\mathrm{MLBS}=98 \\
\mathrm{BPP}=1\end{array}$ \\
\hline & & Liu et al. (2006, fig. 3) & $r p b 1, r p b 2$ & 3 & $\mathrm{BPP}=1$ \\
\hline & & Sugiyama et al. (2007, fig. 2) & LSU, SSU rpb2, $\beta$-tub & 11 & $\mathrm{BPP}=1$ \\
\hline & & Kurtzman \& Sugiyama (2001, fig. 7) & SSU & 8 & $\mathrm{NJBS}=54$ \\
\hline \multirow[t]{3}{*}{ Class/Order } & $\begin{array}{l}\text { Taphrinomycetes, } \\
\text { Taphrinales }\end{array}$ & Sugiyama et al. (2007, fig. 2) & LSU, SSU rpb2, $\beta$-tub & 6 & $\mathrm{BPP}=1$ \\
\hline & & Kurtzman \& Sugiyama (2001, fig. 7) & SSU & 4 & $\mathrm{NJBS}=100$ \\
\hline & & Nishida \& Sugiyama $(1994$, fig. 1) & SSU & 5 & $\mathrm{NJBS}=100$ \\
\hline \multirow[t]{3}{*}{ Class/Order } & $\begin{array}{l}\text { Neolectomycetes, } \\
\text { Neolectales }\end{array}$ & Lutzoni et al. (2004, fig. 2) & LSU, SSU & 1 & NA \\
\hline & & Sugiyama et al. (2007, fig. 2) & LSU, SSU, $\beta$-tub, rpb2 & 2 & $\mathrm{BPP}=1$ \\
\hline & & Landvik et al. (2001, fig. 1) & $\beta$-tub & 2 & MPBS $=100$ \\
\hline \multirow[t]{2}{*}{ Class/Order } & $\begin{array}{l}\text { Pneumocystidomycetes, } \\
\text { Pneumocystidales }\end{array}$ & Sugiyama et al. (2007, fig. 2) & LSU, SSU, $\beta$-tub, rpb2 & 1 & NA \\
\hline & & Lutzoni et al. (2004, fig. 2) & LSU, SSU & 1 & NA \\
\hline \multirow[t]{3}{*}{ Class/Order } & $\begin{array}{l}\text { Schizosaccharomycetes, } \\
\text { Schizosaccharomycetales }\end{array}$ & Sugiyama et al. (2007, fig. 2) & SSU, LSU, rpb2, $\beta$-tub & 1 & NA \\
\hline & & Lutzoni et al. (2004, fig. 2) & LSU, SSU & 2 & $\begin{array}{l}\mathrm{BPP}=1.0 \\
\mathrm{NJBS}=100\end{array}$ \\
\hline & $\begin{array}{l}\text { Taphrinomycotina incertae } \\
\text { sedis (not placed in any } \\
\text { subphylum) }\end{array}$ & & & & \\
\hline \multirow[t]{2}{*}{ Genus } & Saitoella & Sugiyama et al. (2007, fig. 2) & SSU, LSU, rpb2, $\beta$-tub & 1 & NA \\
\hline & & Nishida \& Sugiyama (1994, fig. 1) & SSU & 1 & NA \\
\hline \multirow[t]{2}{*}{ Subphylum/Class/Order } & $\begin{array}{l}\text { Saccharomycotina, } \\
\text { Saccharomycetes, } \\
\text { Saccharomycetales }\end{array}$ & Spatafora et al. (2007, fig. 2) & $\begin{array}{l}\text { LSU, SSU, rpb1, } \\
r p b 2 \text {, tef1 }\end{array}$ & 12 & $\begin{array}{l}\text { WPBS }=55 \\
\mathrm{MLBS}=100 \\
\mathrm{BPP}=1\end{array}$ \\
\hline & & Suh et al. (2007, fig. 2) & LSU, SSU & 87 & $\begin{array}{l}\mathrm{MPBS}=99 \\
\mathrm{BPP}=1\end{array}$ \\
\hline \multirow[t]{3}{*}{ Subphylum } & Pezizomycotina & James et al. (2006, fig. 1) & $\begin{array}{l}\text { SSU, LSU, 5.8S, } \\
\text { rpb1, rpb2, tef1 }\end{array}$ & 46 & $\begin{array}{l}\mathrm{BPP}=1 \\
\mathrm{MLBS}=94\end{array}$ \\
\hline & & Robbertse et al. (2006, figs. 4,5,6) & Genomes & 11 & $\begin{array}{l}\text { MPBS }=94-100 \\
\text { NJBS }=100 \\
\text { MLBS }=100\end{array}$ \\
\hline & & Spatafora et al. (2007, fig. 2) & $\begin{array}{l}\text { LSU, SSU, rpb1, } \\
\text { rpb2, tef1 }\end{array}$ & 157 & $\begin{array}{l}\mathrm{WPBS}=100 \\
\mathrm{MLBS}=97 \\
\mathrm{BPP}=1\end{array}$ \\
\hline \multirow[t]{2}{*}{ Class/Order } & $\begin{array}{l}\text { Arthoniomycetes, } \\
\text { Arthoniales }\end{array}$ & Spatafora et al. (2007, fig. 2) & $\begin{array}{l}\text { LSU, SSU, rpb1, } \\
\text { rpb2, tef1 }\end{array}$ & 4 & $\begin{array}{l}\text { WPBS }=100 \\
\mathrm{MLBS}=100 \\
\mathrm{BPP}=1\end{array}$ \\
\hline & & Lumbsch et al. (2005, fig. 1) & $\begin{array}{l}\text { LSU, SSU, } \\
\text { mt-SSU, mt-LSU }\end{array}$ & 6 & $\begin{array}{l}\mathrm{MPBS}=100 \\
\mathrm{BPP}=1.0\end{array}$ \\
\hline \multirow[t]{3}{*}{ Class } & Dothideomycetes & Schoch et al. (2007, fig.1) & LSU, SSU, rpb2, tef1 & 96 & $\begin{array}{l}\mathrm{BPP}=1 \\
\mathrm{MPBS}<50 \\
\mathrm{MLBS}=70\end{array}$ \\
\hline & & Spatafora et al. (2007, fig. 2) & $\begin{array}{l}\text { LSU, SSU, rpb1, } \\
\text { rpb2, tef1 }\end{array}$ & 17 & $\begin{array}{l}\text { WPBS }<50 \\
\text { MLBS }=84 \\
\text { BPP }=1\end{array}$ \\
\hline & & Kruys et al. (2006, fig. 1) & LSU, SSU, mt-SSU & 51 & $\begin{array}{l}\mathrm{BPP}>0.95 \\
\mathrm{MPBS}<50\end{array}$ \\
\hline Subclass & Dothideomycetidae & Schoch et al. (2007, fig. 1) & LSU, SSU, rpb2, tef1 & 26 & $\begin{array}{l}\mathrm{BPP}=1 \\
\mathrm{MPBS}>50 \\
\mathrm{MLBS}>0.7\end{array}$ \\
\hline
\end{tabular}




\section{Table 2 (continued)}

\begin{tabular}{|c|c|c|c|c|c|}
\hline Rank & Taxon & Reference & Data & OTUs & Support \\
\hline & & Kruys et al. (2006, fig. 1) & LSU, SSU, mt-SSU & 11 & $\mathrm{BPP}>0.95$ \\
\hline & & & & & MPBS $<50$ \\
\hline \multirow[t]{3}{*}{ Order } & Capnodiales & Schoch et al. (2007, fig. 1) & LSU, SSU, rpb2, tef1 & 11 & $\mathrm{BPP}=1$ \\
\hline & & & & & MPBS $>70$ \\
\hline & & & & & MLBS $>70$ \\
\hline \multirow[t]{7}{*}{ Order } & Dothideales & Schoch et al. (2007, fig. 1) & LSU, SSU, rpb2, tef1 & 9 & $\mathrm{BPP}=1$ \\
\hline & & & & & MPBS $>70$ \\
\hline & & & & & MLBS $>70$ \\
\hline & & Kruys et al. (2006, fig. 1) & LSU, SSU, mt-SSU & 4 & $\mathrm{BPP}>0.95$ \\
\hline & & & & & MPBS $=100$ \\
\hline & & Lindemuth et al. (2001) & LSU, SSU & 6 & MLBS $=91$ \\
\hline & & & & & $\mathrm{NJBS}=100$ \\
\hline \multirow[t]{3}{*}{ Order } & Myriangiales & Schoch et al. (2007, fig. 1) & LSU, SSU, rpb2, tef1 & 5 & $\mathrm{BPP}=1$ \\
\hline & & & & & MPBS $>70$ \\
\hline & & & & & MLBS $>70$ \\
\hline \multirow[t]{6}{*}{ Subclass/Order } & Pleosporomycetidae, & Schoch et al. (2007, fig. 1) & LSU, SSU, rpb2, tef1 & 48 & $\mathrm{BPP}=1$ \\
\hline & Pleosporales & & & & MPBS $>70$ \\
\hline & & & & & MLBS $>70$ \\
\hline & & Kruys et al. (2006, fig. 1) & LSU, SSU, mt-SSU & 35 & $\mathrm{BPP}=1$ \\
\hline & & & & & $\mathrm{MPBS}=100$ \\
\hline & $\begin{array}{l}\text { Dothideomycetes } \\
\text { incertae sedis (not placed } \\
\text { in any subclass) }\end{array}$ & & & & \\
\hline \multirow[t]{3}{*}{ Order } & Botryosphaeriales & Schoch et al. (2007, fig. 1) & LSU, SSU, rpb2, tef1 & 8 & $\mathrm{BPP}=1$ \\
\hline & & & & & MPBS $>70$ \\
\hline & & & & & MLBS $>70$ \\
\hline \multirow[t]{3}{*}{ Order } & Hysteriales & Schoch et al. (2007, fig. 1) & LSU, SSU, rpb2, tef1 & 3 & $\mathrm{BPP}=1$ \\
\hline & & & & & MPBS $>70$ \\
\hline & & & & & MLBS $>70$ \\
\hline \multirow[t]{2}{*}{ Order } & Patellariales & Pang et al. (2002, fig. 26) & SSU & 1 & NA \\
\hline & & Inderbitzin et al. (2001, fig. 18) & SSU & 1 & NA \\
\hline Order & Jahnulales & Pang et al. (2002, fig. 26) & SSU & 6 & MPBS $=100$ \\
\hline \multirow[t]{14}{*}{ Class } & Eurotiomycetes & Spatafora et al. (2007, fig. 2) & LSU, SSU, rpb1, & 11 & WPBS $=89$ \\
\hline & & & $r p b 2$, tef1 & & $\mathrm{MLBS}=84$ \\
\hline & & & & & $\mathrm{BPP}=1$ \\
\hline & & Geiser et al. (2007, fig. 1) & SSU, LSU, rpb1, & 49 & $\mathrm{BPP}=1$ \\
\hline & & & rpb2, tef & & MPBS $=100$ \\
\hline & & & & & $\mathrm{WPBS}=100$ \\
\hline & & & & & MLBS $=100$ \\
\hline & & Ekman \& Tønsberg (2002, fig. 1) & SSU & 13 & $\mathrm{BPP}=0.99$ \\
\hline & & Del Prado et al. (2006, fig. 1) & LSU, mt-SSU & 15 & $\mathrm{BPP}=1$ \\
\hline & & Lumbsch et al. (2005, fig. 1) & $\begin{array}{l}\text { LSU, SSU, mt-SSU, } \\
\text { mt-LSU }\end{array}$ & 11 & $\begin{array}{l}\mathrm{BPP}>0.95 \\
\mathrm{MPBS}>70\end{array}$ \\
\hline & & Lutzoni et al. (2004, fig. 5) & LSU, SSU, mt-SSU, rpb2 & 8 & $\mathrm{BPP}=1$ \\
\hline & & & & & $\mathrm{BBS}=61$ \\
\hline & & Reeb et al. (2004, fig. 1) & SSU, LSU, rpb2 & 7 & $\mathrm{BPP}=1$ \\
\hline & & & & & $\mathrm{BBS}=89$ \\
\hline \multirow[t]{15}{*}{ Subclass } & Chaetothyriomycetidae & Reeb et al. (2004, fig. 1) & SSU, LSU, rpb2 & 5 & $\mathrm{BPP}=1$ \\
\hline & & & & & $\mathrm{BBS}=100$ \\
\hline & & & & & MLBS $=100$ \\
\hline & & Lutzoni et al. (2004, fig. 5) & LSU, SSU, & 5 & $\mathrm{BPP}=1$ \\
\hline & & & mt-SSUSSU, rpb2 & & $\mathrm{BBS}=100$ \\
\hline & & & & & $\mathrm{NJBS}=99$ \\
\hline & & & & & MPBS $=98$ \\
\hline & & Del Prado et al. (2006, fig. 1) & LSU, mt-SSU & 11 & $\mathrm{BPP}=1$ \\
\hline & & Spatafora et al. (2007, fig. 1) & SSU, LSU, rpb1, & 6 & $\mathrm{BPP}=1$ \\
\hline & & & $r p b 2$, tef & & MLBS $=100$ \\
\hline & & & & & WPBS $>70$ \\
\hline & & Geiser et al. (2007, fig. 1) & SSU, LSU, & 21 & $\mathrm{BPP}=1$ \\
\hline & & & $r p b 1, r p b 2$, tef & & $\mathrm{MPBS}=100$ \\
\hline & & & & & $\mathrm{WPBBS}=100$ \\
\hline & & & & & MLBS $=100$ \\
\hline \multirow[t]{2}{*}{ Order } & Chaetothyriales & Lutzoni et al. (2004, fig. 2) & LSU, SSU & 5 & $\mathrm{BPP}=1$ \\
\hline & & & & & $\mathrm{NJBS}=94$ \\
\hline
\end{tabular}




\begin{tabular}{|c|c|c|c|c|c|}
\hline Rank & Taxon & Reference & Data & OTUs & Support \\
\hline & & Liu \& Hall (2004, fig. 3) & $r p b 2$ & 5 & $\begin{array}{l}\mathrm{BPP}=1 \\
\mathrm{MPBS}=96\end{array}$ \\
\hline & & Spatafora et al. (2007, fig. 1) & SSU, LSU, rpb1, rpb2, tef & 4 & $\begin{array}{l}\mathrm{BPP}=1 \\
\mathrm{MLBS}=100 \\
\mathrm{WPBS}>70\end{array}$ \\
\hline & & Geiser et al. (2007, fig. 1) & SSU, LSU, rpb1, rpb2, tef & 9 & $\begin{array}{l}\mathrm{BPP}=1 \\
\mathrm{MPBS}=100 \\
\mathrm{WPBBS}=100 \\
\mathrm{MLBS}=100\end{array}$ \\
\hline \multirow[t]{4}{*}{ Order } & Pyrenulales & Lutzoni et al. (2004, fig. 8) & LSU, SSU, mt-SSU, rpb2 & 2 & $\begin{array}{l}\mathrm{BPP}=1 \\
\mathrm{NJBS}=100 \\
\mathrm{WPBS}=100\end{array}$ \\
\hline & & Reeb et al. (2004, fig. 1) & LSU, SSU, rpb2 & 2 & $\begin{array}{l}\mathrm{BPP}=1 \\
\mathrm{BBS}=100 \\
\mathrm{MLBS}=100\end{array}$ \\
\hline & & Schmitt et al. (2004, fig. 1) & LSU, mt-SSU & 2 & $\mathrm{BPP}=1$ \\
\hline & & Geiser et al. (2007, fig. 1) & SSU, LSU, rpb1, rpb2, tef & 5 & $\begin{array}{l}\mathrm{BPP}=1 \\
\mathrm{MPBS}=100 \\
\mathrm{WPBBS}=100 \\
\mathrm{MLBS}=100\end{array}$ \\
\hline \multirow[t]{4}{*}{ Order } & Verrucariales & Wedin et al. (2006, fig. 1) & LSU, mt-SSU & 3 & $\begin{array}{l}\mathrm{BPP}=1 \\
\mathrm{MPjk}=100\end{array}$ \\
\hline & & Geiser et al. (2007, fig. 1) & SSU, LSU, rpb1, rpb2, tef & 7 & $\begin{array}{l}\mathrm{BPP}=1 \\
\mathrm{MPBS}=100 \\
\mathrm{WPBBS}=100 \\
\mathrm{MLBS}=100\end{array}$ \\
\hline & & Lutzoni et al. (2004, fig. 2) & LSU, SSU & 3 & $\begin{array}{l}\mathrm{BPP}=1 \\
\mathrm{NJBS}=98\end{array}$ \\
\hline & & Gueidan et al. (2007, fig. 2) & LSU, SSU, rpb1 & 83 & $\begin{array}{l}\mathrm{BPP}=1 \\
\mathrm{MLBS}=100 \\
\mathrm{MPBS}=100\end{array}$ \\
\hline \multirow[t]{2}{*}{ Subclass } & Eurotiomycetidae & Geiser et al. (2007, fig. 1) & SSU, LSU, rpb1, rpb2, tef & 24 & $\begin{array}{l}\mathrm{BPP}=1 \\
\mathrm{MPBS}=100 \\
\mathrm{WPBBS}=98 \\
\mathrm{MLBS}=100\end{array}$ \\
\hline & & Lutzoni et al. (2004, fig. 2) & LSU, SSU & 11 & $\begin{array}{l}\mathrm{NJBS}=96 \\
\mathrm{BPP}=1\end{array}$ \\
\hline \multirow[t]{3}{*}{ Order } & Coryneliales & Winka (2000, fig. 1) & SSU & 2 & $\begin{array}{l}\text { MPBS }=100 \\
\text { NJBS }=100\end{array}$ \\
\hline & & Inderbitzin et al. (2004, fig. 14) & SSU & 1 & NA \\
\hline & & Geiser et al. (2007, fig. 1) & SSU, LSU, rpb1, rpb2, tef & 3 & $\begin{array}{l}\mathrm{BPP}=1 \\
\mathrm{MPBS}=100 \\
\mathrm{WPBBS}=100 \\
\mathrm{MLBS}=100\end{array}$ \\
\hline Order & Eurotiales & Geiser et al. (2007, fig. 1) & SSU, LSU, rpb1, rpb2, tef & 9 & $\begin{array}{l}\mathrm{BPP}=1 \\
\mathrm{MPBS}=100 \\
W P B B S=100 \\
\mathrm{MLBS}=100\end{array}$ \\
\hline Order & Onygenales & Geiser et al. (2007, fig. 1) & SSU, LSU, rpb1, rpb2, tef & 12 & $\begin{array}{l}\mathrm{BPP}=1 \\
\mathrm{MPBS}=65 \\
\mathrm{WPBBS}=68 \\
\mathrm{MLBS}=88\end{array}$ \\
\hline \multirow[t]{3}{*}{ Subclass/Order } & $\begin{array}{l}\text { Mycocaliciomycetidae, } \\
\text { Mycocaliciales }\end{array}$ & Tibell \& Vinuesa (2005, fig. 1) & LSU & 20 & $\mathrm{BPP}=1$ \\
\hline & & Geiser et al. (2007, fig. 1) & $\begin{array}{l}\text { SSU, LSU, rpb1, } \\
\text { rpb2, tef }\end{array}$ & 4 & $\begin{array}{l}\mathrm{BPP}=1 \\
\mathrm{MPBS}=100 \\
\mathrm{WPBBS}=100 \\
\mathrm{MLBS}=100\end{array}$ \\
\hline & & Ekman \& Tønsberg (2002, fig. 1) & SSU & 4 & $\mathrm{BPP}=1$ \\
\hline \multirow[t]{2}{*}{ Class } & Laboulbeniomycetes & Weir \& Blackwell (2001, fig. 2) & SSU & 4 & MPBS $=100$ \\
\hline & & Henk et al. (2003, fig. 1) & SSU & 6 & MPBS $=100$ \\
\hline \multirow[t]{2}{*}{ Order } & Laboulbeniales & Weir \& Blackwell (2001, fig. 1) & SSU & 3 & MPBS $=100$ \\
\hline & & Henk et al. (2003, fig. 2) & SSU & 3 & MPBS $=57$ \\
\hline \multirow[t]{2}{*}{ Order } & Pyxidiophorales & Weir \& Blackwell (2001, fig. 2) & SSU & 1 & NA \\
\hline & & Henk et al. (2003, fig. 2) & SSU & 2 & MPBS $=99$ \\
\hline
\end{tabular}




\section{Table 2 (continued)}

\begin{tabular}{|c|c|c|c|c|c|}
\hline Rank & Taxon & Reference & Data & OTUs & Support \\
\hline \multirow[t]{4}{*}{ Class } & Lecanoromycetes & Lutzoni et al. (2004, fig. 5) & $\begin{array}{l}\text { LSU, SSU, rpb2, } \\
\text { mt-SSU }\end{array}$ & 34 & $\begin{array}{l}\mathrm{BPP}=1 \\
\mathrm{BBS}=56\end{array}$ \\
\hline & & Spatafora et al. (2007, fig. 2) & $\begin{array}{l}\text { LSU, SSU, rpb1, } \\
\text { rpb2, tef1 }\end{array}$ & 38 & $\begin{array}{l}\text { WPBS }<50 \\
M L B S=93 \\
\text { BPP }=1\end{array}$ \\
\hline & & Miądlikowska et al. (2007, fig. 1) & $\begin{array}{l}\text { LSU, SSU, rpb1, } \\
r p b 2, \mathrm{mt}-\mathrm{SSU}\end{array}$ & 264 & $\begin{array}{l}\text { RMLBS }>70 \\
\text { BS } \\
\text { BPP }>0.95\end{array}$ \\
\hline & & Hofstetter et al. (2007, fig. 1) & $\begin{array}{l}\text { LSU, SSU, rpb1, } \\
r p b 2, \mathrm{mt}-\mathrm{SSU}\end{array}$ & 82 & $\begin{array}{l}\text { RMLBS }>70 \\
\mathrm{BPP}>0.95\end{array}$ \\
\hline \multirow[t]{3}{*}{ Subclass/Order } & $\begin{array}{l}\text { Acarosporomycetidae, } \\
\text { Acarosporales }\end{array}$ & Miądlikowska et al. (2007, fig. 1) & $\begin{array}{l}\text { LSU, SSU, rpb1, } \\
r p b 2, \mathrm{mt}-\mathrm{SSU}\end{array}$ & 15 & $\begin{array}{l}\text { RMLBS }>70 \% \\
\text { PMLBS }>70 \% \\
\text { BPP }>0.95\end{array}$ \\
\hline & & Reeb et al. (2004, fig. 1) & LSU, SSU, rpb2 & 14 & $\begin{array}{l}\mathrm{MLBS}=100 \\
\mathrm{BPP}=100\end{array}$ \\
\hline & & Lutzoni et al. (2004, fig. 4) & LSU, SSU, rpb2 & 14 & $\begin{array}{l}\mathrm{BPP}=1 \\
\mathrm{NJBS}=100 \\
\mathrm{MPBS}=100\end{array}$ \\
\hline \multirow[t]{3}{*}{ Subclass } & Lecanoromycetidae & Miądlikowska et al. (2007, fig. 1) & $\begin{array}{l}\text { LSU, SSU, rpb1, } \\
\text { rpb2, mt-SSU }\end{array}$ & 71 & $\begin{array}{l}\text { RMLBS }>70 \% \\
\text { PMLBS }>70 \% \\
\text { BPP }>0.95\end{array}$ \\
\hline & & Hofstetter et al. (2007, fig. 1) & $\begin{array}{l}\text { LSU, SSU, rpb1, } \\
r p b 2, \mathrm{mt}-\mathrm{SSU}\end{array}$ & 54 & $\begin{array}{l}\text { RMLBS }>70 \\
\mathrm{BPP}>0.95\end{array}$ \\
\hline & & Reeb et al. (2004, fig. 1) & LSU, SSU, rpb2 & 14 & $\begin{array}{l}\mathrm{MLBS}=73 \\
\mathrm{BPP}=100\end{array}$ \\
\hline \multirow[t]{4}{*}{ Order } & Lecanorales & Miądlikowska et al. (2007, fig. 1) & $\begin{array}{l}\text { LSU, SSU, rpb1, } \\
\text { rpb2, mt-SSU }\end{array}$ & 86 & $\begin{array}{l}\text { RMLBS }>70 \\
\text { BS } \\
\text { BPP }>0.95\end{array}$ \\
\hline & & Hofstetter et al. (2007, fig. 1) & $\begin{array}{l}\text { LSU, SSU, rpb1, } \\
r p b 2, \mathrm{mt}-\mathrm{SSU}\end{array}$ & 30 & $\begin{array}{l}\text { RMLBS }>70 \\
\text { BPP }>0.95\end{array}$ \\
\hline & & Lumbsch et al. (2004, fig. 1) & LSU, mt-SSU & 14 & $\mathrm{BPP}=1$ \\
\hline & & Lücking et al. (2004, fig. 3) & LSU, mt-SSU & 8 & $\mathrm{BPP}=1$ \\
\hline \multirow[t]{3}{*}{ Order } & Peltigerales & Miądlikowska et al. (2007, fig. 1) & $\begin{array}{l}\text { LSU, SSU, rpb1, } \\
r p b 2, \mathrm{mt}-\mathrm{SSU}\end{array}$ & 46 & $\begin{array}{l}\text { RMLBS }>70 \\
\text { BSBPP }>0.95\end{array}$ \\
\hline & & Miądlikowska \& Lutzoni (2004, fig. 1) & LSU, SSU & 59 & $\begin{array}{l}\mathrm{MPBS}<70 \\
\mathrm{BPP}=0.92\end{array}$ \\
\hline & & Wilklund \& Wedin (2003, fig. 1) & LSU, SSU & 31 & $\mathrm{Bjk}=99$ \\
\hline Order & Teloschistales & Miądlikowska et al. (2007, fig. 1) & $\begin{array}{l}\text { LSU, SSU, rpb1, } \\
r p b 2, \mathrm{mt}-\mathrm{SSU}\end{array}$ & 13 & $\begin{array}{l}\text { RMLBS }>70 \\
\mathrm{BPP}>0.95\end{array}$ \\
\hline \multirow[t]{3}{*}{ Subclass } & Ostropomycetidae & Miądlikowska et al. (2007, fig. 1) & $\begin{array}{l}\text { LSU, SSU, rpb1, } \\
r p b 2, \mathrm{mt}-\mathrm{SSU}\end{array}$ & 58 & $\begin{array}{l}\text { RMLBS }>70 \\
\mathrm{BS} \\
\mathrm{BPP}>0.95\end{array}$ \\
\hline & & Grube et al. (2004, fig. 1) & mt-SSU & 30 & $\mathrm{BPP}>0.95$ \\
\hline & & Reeb et al. $(2004$, fig. 1$)$ & LSU, SSU, rpb2 & 16 & $\begin{array}{l}\text { MLBS }=100 \\
\mathrm{BPP}=100\end{array}$ \\
\hline \multirow[t]{4}{*}{ Order } & Agyriales & Miądlikowska et al. (2007, fig. 1) & $\begin{array}{l}\text { LSU, SSU, rpb1, } \\
r p b 2, \mathrm{mt}-\mathrm{SSU}\end{array}$ & 8 & $\begin{array}{l}\text { RMLBS }>70 \\
\text { BS } \\
\text { BPP }>0.95\end{array}$ \\
\hline & & Lücking et al. (2004, fig. 3) & LSU, mt-SSU & 11 & $\mathrm{BPP}=1$ \\
\hline & & Lutzoni et al. (2004, fig. 2) & LSU, SSU & 4 & $\begin{array}{l}\mathrm{BPP}=1 \\
\mathrm{NJBS}=100\end{array}$ \\
\hline & & Wedin et al. (2005, fig. 1) & LSU, mt-SSU & 8 & $\begin{array}{l}\mathrm{MPjk}=83 \\
\mathrm{BPP}=0.99\end{array}$ \\
\hline \multirow[t]{2}{*}{ Order } & Baeomycetales & Miądlikowska et al. (2007, fig. 1) & $\begin{array}{l}\text { LSU, SSU, rpb1, } \\
\text { rpb2, mt-SSU }\end{array}$ & 4 & $\begin{array}{l}\text { RMLBS }>70 \\
\text { PMLBS }>70 \\
\text { BPP }>0.95\end{array}$ \\
\hline & & Wedin et al. (2005, fig. 1) & LSU, mt-SSU & 3 & $\begin{array}{l}\mathrm{MPjk}=99 \\
\mathrm{BPP}=1.0\end{array}$ \\
\hline \multirow[t]{3}{*}{ Order } & Ostropales s.l. & Miądlikowska et al. (2007, fig. 1) & $\begin{array}{l}\text { LSU, SSU, rpb1, } \\
r p b 2, \mathrm{mt}-\mathrm{SSU}\end{array}$ & 21 & $\begin{array}{l}\text { RMLBS }>70 \\
\text { BS } \\
\text { BPP }>0.95\end{array}$ \\
\hline & & Schmitt et al. (2005, fig. 1) & LSU, mt-SSU & 12 & $\mathrm{BPP}=1$ \\
\hline & & Wedin et al. (2005, fig. 1) & LSU, mt-SSU & 13 & $\begin{array}{l}\mathrm{Bjk}=94 \\
\mathrm{BPP}=0.97\end{array}$ \\
\hline
\end{tabular}




\begin{tabular}{|c|c|c|c|c|c|}
\hline Rank & Taxon & Reference & Data & OTUs & Support \\
\hline & & Lutzoni et al. (2004, fig. 4) & LSU, SSU, rpb2 & 10 & $\begin{array}{l}\mathrm{BPP}=1 \\
\mathrm{NJBS}=74 \\
\mathrm{MPBS}=84\end{array}$ \\
\hline & & Reeb et al. (2004, fig. 1) & LSU, SSU, rpb2 & 9 & $\begin{array}{l}\mathrm{MLBS}=99 \\
\mathrm{BPP}=1 \\
\mathrm{BBS}=1\end{array}$ \\
\hline \multirow[t]{5}{*}{ Order } & Pertusariales & Miądlikowska et al. (2007, fig. 1) & $\begin{array}{l}\text { LSU, SSU, rpb1, } \\
r p b 2, \mathrm{mt}-\mathrm{SSU}\end{array}$ & 21 & $\begin{array}{l}\text { RMLBS }>70 \\
\text { BSBpp }>0.95\end{array}$ \\
\hline & & Lücking et al. (2004, fig. 3) & LSU, mt-SSU & 7 & $\mathrm{BPP}=1$ \\
\hline & & Schmitt et al. (2005, fig. 1) & LSU, mt-SSU & 14 & $\mathrm{BPP}=1$ \\
\hline & & Lutzoni et al. (2004, fig. 2) & LSU, SSU & 11 & $\mathrm{BPP}=1$ \\
\hline & $\begin{array}{l}\text { Lecanoromycetes incertae } \\
\text { sedis (not placed } \\
\text { in any subclass) }\end{array}$ & & & & \\
\hline \multirow[t]{3}{*}{ Order } & Candelariales & Wedin et al. $(2005$, fig. 1$)$ & LSU, mt-SSU & 3 & $\begin{array}{l}\mathrm{Jk}=100 \\
\mathrm{BPP}=0.96\end{array}$ \\
\hline & & Hofstetter et al. (2007, fig. 1) & LSU, SSU, mt-SSU, rpb1, rpb2 & 2 & $\begin{array}{l}\text { RMLBS }>70 \\
\text { BPP }>0.95\end{array}$ \\
\hline & & Miądlikowska et al. (2007, fig. 1) & LSU, SSU, mt-SSU, rpb1, rpb2 & 3 & $\begin{array}{l}\text { RMLBS }>70 \\
\text { PMLBS }>70 \\
\text { BPP }>0.95\end{array}$ \\
\hline \multirow[t]{3}{*}{ Order } & Umbilicariales & $\begin{array}{l}\text { Miądlikowska et al. (2007, fig. 1) } \\
\text { Miądlikowska et al. (2007, fig. 1) }\end{array}$ & $\begin{array}{l}\text { LSU, SSU, rpb1, rpb2, mt-SSU } \\
\text { LSU, SSU, rpb1, rpb2, mt-SSU }\end{array}$ & $\begin{array}{r}16 \\
9\end{array}$ & $\begin{array}{l}\text { BSBSBPP }>0.95 \\
\text { RMLBS }>70 \\
\text { PMLBS }>70 \\
\text { BPP }>0.95\end{array}$ \\
\hline & & Hofstetter et al. (2007, fig. 1) & LSU, SSU, rpb1, rpb2, mt-SSU & 8 & $\begin{array}{l}\text { RMLBS }>70 \\
\text { BPP }>0.95\end{array}$ \\
\hline & & Reeb et al. (2004, fig. 1) & LSU, SSU, rpb2 & 4 & $\begin{array}{l}\mathrm{MLBS}=70 \\
\mathrm{BPP}=1 \\
\mathrm{BBS}=88\end{array}$ \\
\hline \multirow[t]{3}{*}{ Class } & $\begin{array}{l}\text { Leotiomycetes } \\
\text { (w/o Geoglossaceae) }\end{array}$ & Spatafora et al. (2007, fig. 2) & LSU, SSU, rpb1, rpb2, tef1 & 22 & $\begin{array}{l}\mathrm{WPBS}=100 \\
\mathrm{MLBS}=100 \\
\mathrm{BPP}=1\end{array}$ \\
\hline & & Wang et al. (2006, fig. 1) & LSU, SSU, 5.8S & 50 & $\mathrm{BPP}=1$ \\
\hline & & Wang et al. (2007, fig. 2) & LSU, SSU, $5.8 \mathrm{~S}$ & 78 & $\begin{array}{l}\mathrm{MPBS}=61 \\
\mathrm{BPP}=1\end{array}$ \\
\hline Order & Cyttariales & Wang et al. (2007, fig. 1) & SSU, LSU, $5.8 \mathrm{~S}$ & 1 & NA \\
\hline \multirow[t]{3}{*}{ Order } & Erysiphales & Rossman et al. (2004, fig. 2) & LSU & 12 & MPBS $>55$ \\
\hline & & Wang et al. (2007, fig. 1) & SSU, LSU, 5.8S & 16 & $\begin{array}{l}\mathrm{MPBS}=63 \\
\mathrm{BPP}=0.97\end{array}$ \\
\hline & & Takamatsu (2004, fig. 2) & SSU & 10 & $\mathrm{NJBS}=99$ \\
\hline Order & $\begin{array}{l}\text { Helotiales } \\
\text { (w/o Geoglossaceae) }\end{array}$ & Wang et al. (2007, fig. 1) & SSU, LSU, 5.8S & 40 & $\mathrm{BPP}<0.90$ \\
\hline \multirow[t]{2}{*}{ Order } & Rhytismatales & Rossman et al. (2004, fig. 2) & LSU & 4 & MPBS $>55$ \\
\hline & & Wang et al. (2007, fig. 1) & SSU, LSU, $5.8 \mathrm{~S}$ & 5 & $\begin{array}{l}\mathrm{MPBS}=100 \\
\mathrm{BPP}=1\end{array}$ \\
\hline Order & Thelebolales & de Hoog et al. (2005, fig. 3) & SSU & 11 & MPBS $=56$ \\
\hline \multirow[t]{3}{*}{ Class/Order } & $\begin{array}{l}\text { Lichinomycetes, } \\
\text { Lichinales }\end{array}$ & Spatafora et al. (2007, fig. 2) & LSU, SSU, rpb1, rpb2, tef1 & 1 & NA \\
\hline & & Miądlikowska et al. (2007, fig. 1) & $\begin{array}{l}\text { LSU, SSU, rpb1, } \\
r p b 2, \mathrm{mt}-\mathrm{SSU}\end{array}$ & 2 & $\begin{array}{l}\text { RMLBS }>70 \\
\text { PMLBS }>70 \\
\text { BPP }>0.95\end{array}$ \\
\hline & & Reeb et al. (2004, fig. 1) & LSU, SSU, rpb2 & 3 & $\begin{array}{l}\mathrm{MLBS}=100 \\
\mathrm{BBS}=100 \\
\mathrm{BPP}=1\end{array}$ \\
\hline Class/Order & $\begin{array}{l}\text { Orbiliomycetes, } \\
\text { Orbiliales }\end{array}$ & Spatafora et al. (2007, fig. 2) & LSU, SSU, rpb1, rpb2, tef1 & 2 & $\begin{array}{l}\mathrm{WPBS}=100 \\
\mathrm{MLBS}=100 \\
\mathrm{BPP}=1\end{array}$ \\
\hline \multirow[t]{2}{*}{ Class/Order } & $\begin{array}{l}\text { Pezizomycetes, } \\
\text { Pezizales }\end{array}$ & Spatafora et al. (2007, fig. 2) & LSU, SSU, rpb1, rpb2, tef1 & 14 & $\begin{array}{l}\mathrm{WPBS}=54 \\
\mathrm{MLBS}=99 \\
\mathrm{BPP}=1\end{array}$ \\
\hline & & Lutzoni et al. (2004, fig. 2) & LSU, SSU & 21 & $\begin{array}{l}\mathrm{BPP}=0.96 \\
\mathrm{NJBS}=70\end{array}$ \\
\hline Class & Sordariomycetes & Spatafora et al. (2007, fig. 2) & LSU, SSU, rpb1, rpb2, tef1 & 47 & $\begin{array}{l}\mathrm{WPBS}=100 \\
\mathrm{MLBS}=100 \\
\mathrm{BPP}=1\end{array}$ \\
\hline
\end{tabular}




\section{Table 2 (continued)}

\begin{tabular}{|c|c|c|c|c|c|}
\hline Rank & Taxon & Reference & Data & OTUs & Support \\
\hline & & Zhang et al. (2007, fig. 2) & LSU, SSU, rpb2, tef1 & 106 & $\begin{array}{l}\mathrm{MPBS}=100 \\
\mathrm{WPBS}=100 \\
\mathrm{MLBS}=100 \\
\mathrm{BPP}=1\end{array}$ \\
\hline & & Lutzoni et al. (2004, fig. 2) & LSU, SSU & 66 & $\begin{array}{l}\mathrm{BPP}=1 \\
\mathrm{NJBS}=97\end{array}$ \\
\hline \multirow[t]{3}{*}{ Subclass } & Hypocreomycetidae & Zhang et al. (2007, fig. 2) & LSU, SSU, rpb2, tef1 & 42 & $\begin{array}{l}\mathrm{MPBS}=92 \\
\mathrm{WPBS}=96 \\
\mathrm{MLBS}=90 \\
\mathrm{BPP}=1\end{array}$ \\
\hline & & Lutzoni et al. (2004, fig. 2) & LSU, SSU & 26 & $\begin{array}{l}\mathrm{NJBS}<50 \\
\mathrm{BPP}=1\end{array}$ \\
\hline & & Huhndorf et al. (2004b, figs. 38, 39) & LSU & 21 & $\begin{array}{l}\mathrm{MPBS}=67 \\
\mathrm{BPP}>=0.95\end{array}$ \\
\hline \multirow[t]{3}{*}{ Order } & Coronophorales & Zhang et al. (2007, fig. 2) & LSU, SSU, rpb2, tef1 & 2 & $\begin{array}{l}\mathrm{MPBS}<50 \\
\mathrm{WPBS}<50 \\
\mathrm{MLBS}=96 \\
\mathrm{BPP}=1\end{array}$ \\
\hline & & Huhndorf et al. (2004b, figs. 38, 39) & LSU & 16 & $\begin{array}{l}\text { WPBS }=99 \\
\text { BPP } \geq 95\end{array}$ \\
\hline & & Miller \& Huhndorf (2005, fig. 7) & LSU, $\beta$-tub, rpb2 & 2 & $\begin{array}{l}\text { WPBS }=100 \\
\text { BPP } \geq 95\end{array}$ \\
\hline \multirow[t]{2}{*}{ Order } & Hypocreales & Zhang et al. (2007, fig. 2) & LSU, SSU, rpb2, tef1 & 21 & $\begin{array}{l}\mathrm{MPBS}=91 \\
\mathrm{WPBS}=90 \\
\mathrm{MLBS}=72 \\
\mathrm{BPP}=1\end{array}$ \\
\hline & & Castlebury et al. (2004, fig. 1) & LSU, SSU & 31 & $\begin{array}{l}\mathrm{MPBS}=70 \\
\mathrm{BPP}=1\end{array}$ \\
\hline Order & Melanosporales & Zhang et al. (2007, fig. 2) & LSU, SSU, rpb2, tef1 & 2 & $\begin{array}{l}\mathrm{MPBS}=100 \\
\mathrm{WPBS}=100 \\
\mathrm{MLBS}=100 \\
\mathrm{BPP}=1\end{array}$ \\
\hline \multirow[t]{4}{*}{ Order } & $\begin{array}{l}\text { Microascales } \\
\text { (incl. Halosphaeriales) }\end{array}$ & Zhang et al. (2007, fig. 2) & LSU, SSU, rpb2, tef1 & 15 & $\begin{array}{l}\mathrm{MPBS}=74 \\
\mathrm{WPBS}=86 \\
\mathrm{MLBS}=85 \\
\mathrm{BPP}=1\end{array}$ \\
\hline & & Lutzoni et al. (2004, fig. 2) & LSU, SSU & 10 & $\begin{array}{l}\mathrm{NJBS}=80 \\
\mathrm{BPP}=1\end{array}$ \\
\hline & & Campbell et al. (2003, fig. 3) & LSU, SSU & 40 & $\begin{array}{l}\mathrm{MPBS}=100 \\
\mathrm{BPP}=1\end{array}$ \\
\hline & & Kohlmeyer et al. (2000, fig. 1) & LSU, SSU & 16 & MPBS $=97$ \\
\hline \multirow[t]{2}{*}{ Subclass } & Sordariomycetidae & Zhang et al. (2007, fig. 2) & LSU, SSU, rpb2, tef1 & 54 & $\begin{array}{l}\mathrm{MPBS}=82 \\
\mathrm{WPBS}=85 \\
\mathrm{MLBS}=77 \\
\mathrm{BPP}=1\end{array}$ \\
\hline & & Lutzoni et al. (2004, fig. 2) & LSU, SSU & 36 & $\begin{array}{l}\mathrm{NJBS}<50 \\
\mathrm{BPP}=0.97\end{array}$ \\
\hline \multirow[t]{3}{*}{ Order } & Boliniales & Zhang et al. (2007, fig. 2) & LSU, SSU, rpb2, tef1 & 4 & $\begin{array}{l}\mathrm{MPBS}=100 \\
\mathrm{WPBS}=100 \\
\mathrm{MLBS}=100 \\
\mathrm{BPP}=1\end{array}$ \\
\hline & & Huhndorf et al. (2004a, fig. 1) & LSU & 3 & $\begin{array}{l}\text { WPBS }=99 \\
\mathrm{BPP}<95\end{array}$ \\
\hline & & Miller \& Huhndorf (2005, fig. 7) & LSU, $\beta$-tub, rpb2 & 2 & $\begin{array}{l}\text { WPBS }=100 \\
\mathrm{BPP} \geq 95\end{array}$ \\
\hline \multirow[t]{3}{*}{ Order } & Chaetosphaeriales & Zhang et al. (2007, fig. 2) & LSU, SSU, rpb2, tef1 & 3 & $\begin{array}{l}\mathrm{MPBS}=100 \\
\mathrm{WPBS}=100 \\
\mathrm{MLBS}=100 \\
\mathrm{BPP}=1\end{array}$ \\
\hline & & Miller \& Huhndorf (2005, fig. 7) & LSU, $\beta$-tub, rpb2 & 2 & $\begin{array}{l}\text { WPBS }=100 \\
B P P \geq 95\end{array}$ \\
\hline & & Shenoy et al. (2006, fig. 3) & LSU, $r p b 2$ & $\begin{array}{r}4 \\
\text { (contin }\end{array}$ & $\begin{array}{l}\text { MPBS }=100 \\
\text { ued on next pac }\end{array}$ \\
\hline
\end{tabular}




\begin{tabular}{|c|c|c|c|c|c|}
\hline Rank & Taxon & Reference & Data & OTUs & Support \\
\hline \multirow[t]{3}{*}{ Order } & Coniochaetales & Zhang et al. (2007, fig. 2) & LSU, SSU, rpb2, tef1 & 3 & $\begin{array}{l}\mathrm{MPBS}=93 \\
\mathrm{WPBS}=100 \\
\mathrm{MLBS}=87 \\
\mathrm{BPP}=1\end{array}$ \\
\hline & & Miller \& Huhndorf (2005, fig. 7) & LSU, $\beta$-tub, rpb2 & 2 & $\begin{array}{l}\text { WPBS }=100 \\
B P P \geq 95\end{array}$ \\
\hline & & Miller \& Huhndorf (2004, fig. 10) & LSU & 3 & $\begin{array}{l}\text { WPBS }=98 \\
\mathrm{BPP} \geq 95\end{array}$ \\
\hline \multirow[t]{5}{*}{ Order } & Diaporthales & Zhang et al. (2007, fig. 2) & LSU, SSU, rpb2, tef1 & 19 & $\begin{array}{l}\mathrm{MPBS}=95 \\
\mathrm{WPBS}=94 \\
\mathrm{MLBS}=77 \\
\mathrm{BPP}=1\end{array}$ \\
\hline & & Castlebury et al. (2002, fig. 1) & LSU & 82 & $\begin{array}{l}\mathrm{MPBS}=100 \\
\mathrm{NJBS}=100\end{array}$ \\
\hline & & Lutzoni et al. (2004, fig. 2) & LSU, SSU & 10 & $\begin{array}{l}\mathrm{NJBS}=100 \\
\mathrm{BPP}=1\end{array}$ \\
\hline & & Miller \& Huhndorf (2005, fig. 7) & LSU, $\beta$-tub, rpb2 & 2 & $\begin{array}{l}\text { WPBS }=100 \\
\text { BPP } \geq 95\end{array}$ \\
\hline & & Miller \& Huhndorf (2004, fig. 10) & LSU & 3 & $\begin{array}{l}\mathrm{WPBS}=100 \\
\mathrm{BPP}=\geq 95\end{array}$ \\
\hline \multirow[t]{3}{*}{ Order } & Ophiostomatales & Zhang et al. (2007, fig. 2) & LSU, SSU, rpb2, tef1 & 3 & $\begin{array}{l}\mathrm{MPBS}=100 \\
\mathrm{WPBS}=100 \\
\mathrm{MLBS}=100 \\
\mathrm{BPP}=1\end{array}$ \\
\hline & & Hausner \& Reid (2004, fig. 1) & SSU & 3 & $\mathrm{NJBS}=99$ \\
\hline & & Wingfield et al. (1999, fig. 3) & LSU & 4 & MPBS $=99$ \\
\hline \multirow[t]{3}{*}{ Order } & Sordariales & Zhang et al. (2007, fig. 2) & LSU, SSU, rpb2, tef1 & 17 & $\begin{array}{l}\mathrm{MPBS}=80 \\
\mathrm{WPBS}=77 \\
\mathrm{MLBS}=84 \\
\mathrm{BPP}=1\end{array}$ \\
\hline & & Huhndorf et al. (2004a, fig. 1) & LSU & 22 & $\begin{array}{l}\mathrm{WPBS}=<50 \\
\mathrm{BPP}<95\end{array}$ \\
\hline & & Miller \& Huhndorf (2005, fig. 7) & LSU, $\beta$-tub, rpb2 & 41 & $\begin{array}{l}\text { WPBS }=65 \\
\mathrm{BPP} \geq 95\end{array}$ \\
\hline \multirow[t]{2}{*}{ Subclass/Order } & $\begin{array}{l}\text { Xylariomycetidae, } \\
\text { Xylariales }\end{array}$ & Zhang et al. (2007, fig. 2) & LSU, SSU, rpb2, tef1 & 8 & $\begin{array}{l}\mathrm{MPBS}=98 \\
\mathrm{WPBS}=99 \\
\mathrm{MLBS}=78 \\
\mathrm{BPP}=1\end{array}$ \\
\hline & $\begin{array}{l}\text { Sordariomycetes incertae } \\
\text { sedis (not placed in } \\
\text { any subclass) }\end{array}$ & Shenoy et al. (2006, fig. 1) & LSU & 16 & $\mathrm{MPBS}=92$ \\
\hline \multirow[t]{3}{*}{ Order } & Calosphaeriales & Vijaykrishna et al. (2004, fig. 1) & SSU & 3 & MPBS $=100$ \\
\hline & & Réblová et al. (2004, fig. 1) & LSU & 6 & MPBS $=53$ \\
\hline & & Réblová (2006, fig. 1) & SSU & 2 & MPBS $=68$ \\
\hline \multirow[t]{4}{*}{ Order } & $\begin{array}{l}\text { Lulworthiales } \\
\text { (incl. Spathulosporales) }\end{array}$ & Zhang et al. (2007, fig. 2) & LSU, SSU, rpb2, tef1 & 2 & $\begin{array}{l}\mathrm{MPBS}=100 \\
\mathrm{WPBS}=100 \\
\mathrm{MLBS}=100 \\
\mathrm{BPP}=1\end{array}$ \\
\hline & & Campbell et al. (2005, fig. 1) & LSU, SSU & 56 & $\mathrm{BPP}=1$ \\
\hline & & Inderbitzin et al. (2004, fig. 15) & LSU & 15 & $\begin{array}{l}\mathrm{MPBS}=100 \\
\mathrm{NJBS}=91 \\
\mathrm{BPP}=86\end{array}$ \\
\hline & & Kohlmeyer et al. (2000, fig. 1) & LSU, SSU & 7 & MPBS $=100$ \\
\hline Order & Meliolales & Saenz \& Taylor (1999, fig. 1) & LSU & 2 & MPBS $=100$ \\
\hline Order & & Vijaykrishna et al. (2004, fig. 1) & SSU & 2 & MPBS $<50$ \\
\hline Order & Phyllachorales & Inderbitzin et al. (2004, fig. 14) & SSU & 1 & NA \\
\hline Order & $\begin{array}{l}\text { Trichosphaeriales } \\
\text { Pezizomycotina incertae } \\
\text { sedis (not placed } \\
\text { in any class) }\end{array}$ & Réblová \& Seifert (2004, fig. 1) & LSU & 8 & MPBS $<50$ \\
\hline Order & Lahmiales & Eriksson (1986) & - & - & - \\
\hline Order & Medeolariales & Inderbitzin et al. (2004, fig. 14) & SSU & 1 & NA \\
\hline Order & Triblidiales & Eriksson (1992) & - & - & - \\
\hline
\end{tabular}




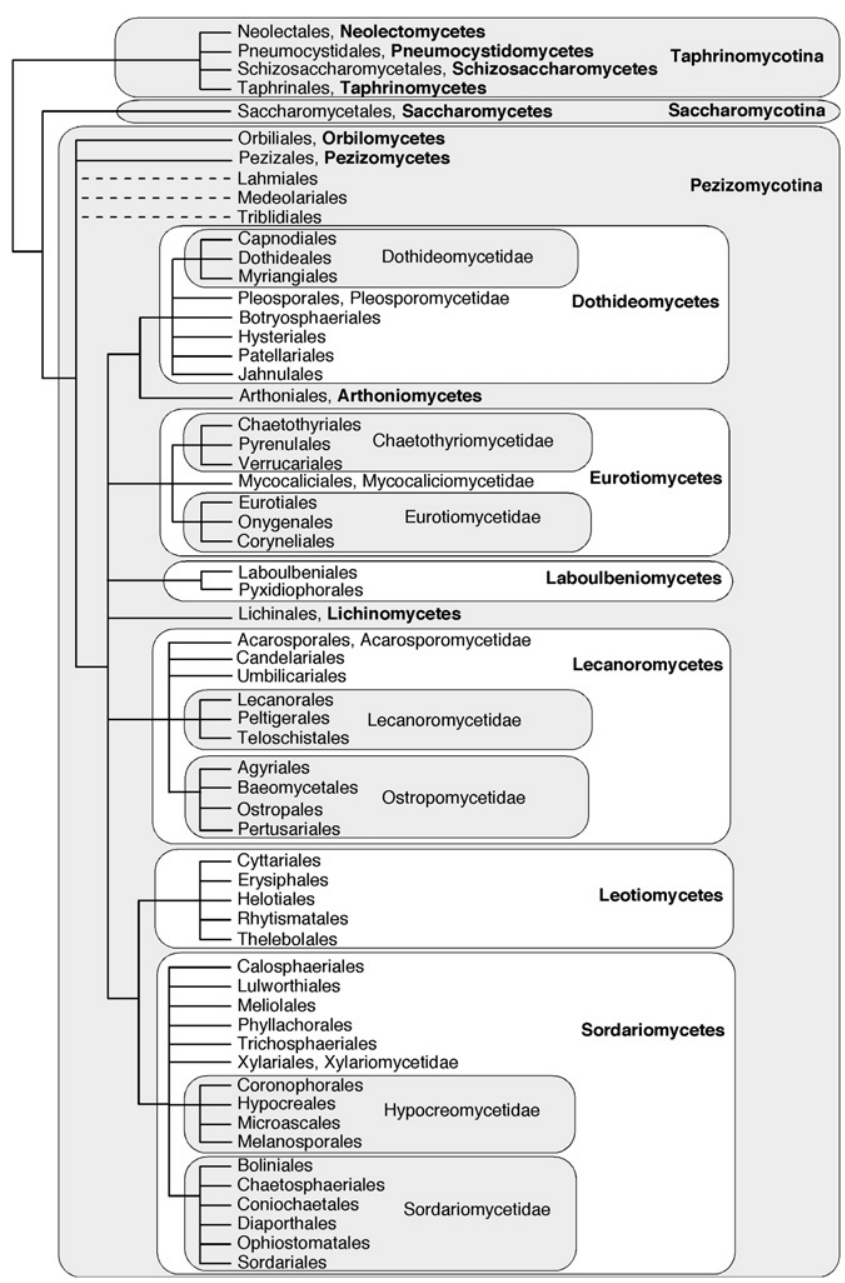

Fig 2 - Phylogeny and classification of Fungi. Ascomycota. See Table 2 for support values for clades. Dashed lines indicate taxa that are of uncertain placement.

Subclass: Dothideomycetidae P. M. Kirk, P. F. Cannon, J. C. David \& Stalpers ex Schoch et al., Mycologia 98: 1047 (2007) ['2007'].

Order: Capnodiales Woron., Annls Mycol. 23: 177 (1925).

Exemplar genera: Capnodium Mont. 1848, Scorias Fr. 1825, Mycosphaerella Johanson 1884.

Order: Dothideales Lindau, in Engler \& Prantl (eds), Nat. Pflanzenfam. 1(1): 373 (1897).

Exemplar genera: Dothidea Fr. 1818, Dothiora Fr. 1849, Sydowia Bres. 1895, Stylodothis Arx \& E. Müll. 1975.

Order: Myriangiales Starbäck, K. suenska Vetensk-Akad. Handl., Bih., Afd. III 25: 37 (1899).

Exemplar genera: Myriangium Mont. \& Berk. 1845, Elsinoё Racib. 1900.

Subclass: Pleosporomycetidae C. L. Schoch, Spatafora, Crous \& Shoemaker, Mycologia 98: 1049 (2007) ['2006'].
Order: Pleosporales Luttr. ex M. E. Barr, Prodr. Class Loculoasc.: 67 (1987b).

Synonym: Pleosporales Luttr., Mycologia 47: 520 (1955), nomen invalidum.

Exemplar genera: Pleospora Rabenh. ex Ces. \& De Not. 1863, Phaeosphaeria I. Miyake 1909, Lophiostoma Ces. \& De Not. 1863, Sporormiella Ellis \& Everh.1892, Montagnula Berl. 1896.

Dothideomycetes incertae sedis (not placed in any subclass)

Order: Botryosphaeriales C. L. Schoch, Crous \& Shoemaker, Mycologia 98: 1051 (2007) ['2006'].

Exemplar genera: Botryosphaeria Ces. \& De Not. 1863, Guignardia Viala \& Ravaz 1892.

Order: Hysteriales Lindau in Engler \& Prantl (eds), Nat. Pflanzenfam. 1: 265 (1896), as 'Hysteriinae'.

Exemplar genera: Hysterium Pers. 1797, Hysteropatella Rehm. 1890.

Order: Patellariales D. Hawksw. \& O. E. Erikss., Syst. Ascom. 5: 181 (1986).

Exemplar genus: Patellaria Fr. 1822.

Order: Jahnulales Ka-Lai Pang, Abdel-Wahab, El-Shar., E. B. G. Jones \& Sivichai, in Pang et al., Mycol. Res. 106: 1033 (2002).

Exemplar genera: Aliquandostipite Inderb. 2001, Jahnula Kirschst. 1936, Patescospora Abdel-Wahab \& El-Shar. 2002.

Class: Eurotiomycetes O. E. Erikss. \& Winka, Myconet 1: 6 (1997).

The circumscription of this class and the classification within the Eurotiomycetes presented here are derived from the phylogenetic re-delimitation of this class by Ekman \& Tønsberg (2002), Lutzoni et al. (2004) and Geiser et al. (2007), reflecting the inference of shared ancestry between Eurotiomycetes, comprising Coryneliales, Onygenales and Eurotiales and Chaetothyriomycetes. Three subclasses, Chaetothyriomycetidae, Eurotiomycetidae, and Mycocaliciomycetidae, are defined to represent the major lineages within Eurotiomycetes.

Subclass: Chaetothyriomycetidae Doweld, Prosyllabus: LXXVIII (2001).

Lichenized, parasitic, and saprobic ascomycetes with mostly bitunicate/fissitunicate to evanescent asci, produced in perithecial ascomata arranged superficially or immersed in a thallus. Thalli often produced on the surfaces of rocks, lichens, decaying plant material and other substrata. Ascospores variable, from colourless to pigmented, simple to muriform. Hamathecium, when present, consisting of pseudoparaphyses. Pigments, when present, generally related to melanin. Asexual stages with phialidic and annellidic anamorphs observed in non-lichenized taxa.

Order: Chaetothyriales M. E. Barr, Mycotaxon 29: 502 (1987).

Exemplar genera: Capronia Sacc. 1883, Ceramothyrium Bat. \& H. Maia 1956, Chaetothyrium Speg. 1888. 
Order: Pyrenulales Fink ex D. Hawksw. \& O. E. Erikss., Syst. Ascom. 5: 182 (1986).

Synonym: Pyrenulales Fink, Ohio St. Univ. Bull. 19(28): 107 (1951), nomen invalidum.

Exemplar genera: Pyrenula Ach. 1814, Pyrgillus Nyl. 1858.

Order: Verrucariales Mattick ex D. Hawksw. \& O. E. Erikss., Syst. Ascom. 5: 183 (1986).

Synonym: Verrucariales Mattick, in Engler, Syll. Pflanzenfam. (12 edn): 208 (1954), nomen invalidum.

Exemplar genera: Agonimia Zahlbr. 1909, Dermatocarpon Eschw. 1824, Polyblastia A. Massal. 1852, Verrucaria Schrad. 1794.

Subclass: Eurotiomycetidae Geiser \& Lutzoni, subclass. nov. MycoBank no.: MB 501287

Fungi saprotrophici vel parasitici vel mycorrhizales; asci globosi in toto ascomate sparsi, raro hymenium formantes; asci plerumque evanescentes, nonnumquam bitunicati. Ascosporae plerumque unicellulares, lenticulares, nonnumquam globosae vel ellipsoideae. Ascomata, si formata, plerumque cleistothecialia vel gymnothecialia, saepe textura stromatica circumdata. Structurae hamatheciales absentes. Gametangia plerumque indistincta e glomere hyphali constantia. Fungi saepe laete colorati. Anamorphae variabiles, seu phialidicae seu arthroconidiales.

Typus: Eurotium Link 1809.

Saprotrophic, parasitic and mycorrhizal. Ascomata, when present, usually cleistothecial/gymnothecial, globose, often produced in surrounding stromatic tissue and brightly coloured; hamathecial elements lacking; gametangia usually undifferentiated and consisting of hyphal coils. Asci usually evanescent, sometimes bitunicate, scattered throughout the ascoma, rarely from a hymenium. Ascospores usually single-celled, lenticular, sometimes spherical or elliptical. Anamorphs variable, including phialidic and arthroconidial forms.

This name was employed by Lutzoni et al. (2004) and Geiser et al. (2007), in the same sense as the present classification, but without a formal diagnosis.

Order: Coryneliales Seaver \& Chardón, Scient. Surv. P. Rico: 40 (1926).

Exemplar genera: Corynelia Ach. 1823, Caliciopsis Peck 1880.

Order: Eurotiales G. W. Martin ex Benny \& Kimbr., Mycotaxon 12: 23 (1980).

Synonym: Eurotiales G. W. Martin, Std. nat. Hist. Iowa Univ. 18(Suppl.): 16 (1941), nomen invalidum.

Exemplar genera: Eurotium Link 1809, Emericella Berk. 1857, Talaromyces C. R. Benj. 1955, Elaphomyces Nees 1820, Trichocoma Jungh. 1838, Byssochlamys Westling 1909.

Order: Onygenales Cif. ex Benny \& Kimbr., Mycotaxon 12: 8 (1980). Synonym: Onygenales Cif., Atti Ist. Bot. Univ. Pavia, ser. 5, 14: 238

(1957), nomen invalidum.

Emend. Currah Mycotaxon 24: 13 (1985).

Exemplar genera: Onygena Pers. 1799, Gymnoascus Baran. 1872, Arthroderma Curr. 1860.

Subclass: Mycocaliciomycetidae Tibell. subclass nov. MycoBank no.: MB 501288
Parasitae vel commensales in lichenibus vel saprotrophici. Ascomata disciformia, stipitata vel sessilia. Excipulum cupulatum, saltem partim scleroticum hyphis stipitis simile. Dispersio sporarum activa, raro passiva et tum mazedio parce evoluto. Asci unitunicati, cylindrici, vulgo apice distincte incrassato, 8-spori. Ascosporae pallidae ad atrofuscae, ellipsoidales, nonseptatae vel transversaliter 1-7-septatae. Paries sporae atrofuscus, laevis vel ornamento intra plasmalemma formato. Derivata acidi vulpinici in speciebus paucis praesentia. Anamorphae coelomycetum et hyphomycetum variae praesentes.

Typus: Mycocalicium Vain. 1890.

Parasites or commensals on lichens or saprobes. Ascomata disciform, stalked or sessile. Excipulum cupulate, and like the stalk hyphae at least in part sclerotized. Spore dispersal active, more rarely passive and ascomata then with a moderately developed mazaedium. Asci unitunicate, cylindrical, mostly with a distinctly thickened apex, 8-spored. Ascospores pale to blackish brown, ellipsoidal or spherical to cuboid, non-septate or transversely 1-7-septate. Spore wall pigmented, smooth or with an ornamentation formed within the plasmalemma. Vulpinic acid derivatives occur in a few species. A variety of coelomycetous and hyphomycetous anamorphs occur.

Order: Mycocaliciales Tibell \& Wedin, Mycologia 92: 579 (2000). Exemplar genera: Mycocalicium Vain. 1890, Chaenothecopsis Vain. 1927, Stenocybe (Nyl.) Körb. 1855, Sphinctrina Fr. 1825.

Class: Laboulbeniomycetes Engl., Syll. Pflanzenfam. (2nd edn): 46 (1898).

Order: Laboulbeniales Lindau, in Engler \& Prantl (eds), Nat. Pflanzenfam. 1(1): 491 (1897), as 'Laboulbeniineae'.

Exemplar genera: Laboulbenia Mont. \& C.P. Robin 1835, Rickia Cavara 1899, Ceratomyces Thaxt. 1892.

Order: Pyxidiophorales P. F. Cannon, in Kirk et al., Ainsworth \& Bisby's Dict. Fungi (9th edn): xi (2001).

Exemplar genus: Pyxidiophora Bref. \& Tavel 1891.

Class: Lecanoromycetes O. E. Erikss. \& Winka, Myconet 1: 7 (1997).

Subclass: Acarosporomycetidae Reeb, Lutzoni \& Cl. Roux, Mol. Phylogen. Evol. 32: 1053 (2004).

Order: Acarosporales Reeb, Lutzoni \& Cl. Roux, ord. nov. MycoBank no.: MB 501289

Ascomycetes lichenisati algas virides thallo continentes. Ascomata immersa vel sessilia, disciformia vel perithecioidea. Excipulum hyalinum, annulatum. Hymenium non-amyloideum. Paraphyses mediocriter vel infirme ramosae, septatae, mediocriter vel infirme anastomosantes. Asci unitunicati, non-amyloidei vel satis infirme amyloidei, polyspori. Ascosporae hyalinae, non-septatae, nonhalonatae.

Typus: Acarospora A. Massal. 1852.

Lichen-forming ascomycetes with chlorococcoid photobiont. Ascomata immersed or sessile, disciform or perithecioid. True exciple hyaline, annulate. Hymenium non- 
amyloid. Paraphyses moderately to poorly branched, septate, moderately to poorly anastomosing. Asci functionally unitunicate, lecanoralean, non-amyloid or with slightly amyloid tholi, polyspored, generally with more than 100 ascospores per ascus. Ascospores hyaline, small, non-septate, non-halonate.

The members of this order were formerly classified within the Lecanorales, but Reeb et al. (2004) and Lutzoni et al. (2004) demonstrated that the Acarosporaceae diverged earlier than the Lecanoromycetidae and Ostropomycetidae. This early divergence within the Lecanoromycetes was confirmed by Wedin et al. (2005) and Miądlikowska et al. (2007).

Exemplar genera: Acarospora A. Massal. 1852, Pleopsidium Körb. 1855, Sarcogyne Flot. 1851.

Subclass: Lecanoromycetidae P. M. Kirk, P. F. Cannon, J. C. David \& Stalpers ex Miądl., Lutzoni \& Lumbsch, subclass. nov. MycoBank no.: MB 501290

Synonym: Lecanoromycetidae P. M. Kirk, P. F. Cannon, J. C. David \& Stalpers, Ainsworth \& Bisby's Dict. Fungi (9th edn): xi (2001), nomen invalidum.

Ascomycetes lichenisati algas virides vel cyanobacteria thallo continentes. Ascomata immersa, sessilia vel elevata, generaliter disciformia. Excipulum hyalinum vel pigmentatum, annulatum vel cupulatum. Hymenium amyloideum vel non-amyloideum. Paraphyses simplices vel ramosae, septatae, anastomosantes vel non-anastomosantes. Asci bitunicati, unitunicati vel prototunicati, non-amyloidei vel amyloidei, generaliter octospori, sed etiam 1- ad multispori. Ascosporae hyalinae vel brunneae, non-septatae, vel septate usque ad muriformes, halonatae vel non-halonatae.

Typus: Lecanora Ach. 1809.

Lichen-forming ascomycetes with green algal or cyanobacterial photobiont. Ascomata immersed, sessile or stalked, usually disciform. True exciple hyaline or pigmented, annulate or cupulate. Hymenium amyloid or non-amyloid. Paraphyses simple or moderately to richly branched, septate, anastomosing or not. Asci bitunicate, functionally unitunicate, or prototunicate, lecanoralean, non-amyloid or amyloid, mostly 8-spored, but varying from 1- to poly-spored. Ascospores hyaline or brown, non-septate, trans-septate or muriform, halonate or non-halonate.

This subclass includes the bulk of lichenized discomycetes and corresponds to the phylogenetic circumscription of this subclass by Reeb et al. (2004), Lutzoni et al. (2004) and Miądlikowska et al. (2007). It is in agreement with the Lecanorales of Lumbsch et al. (2004) and Wiklund \& Wedin (2004). The orders Peltigerales and Teloschistales are here accepted at the ordinal level, following Miądlikowska \& Lutzoni (2003) and Miądlikowska et al. (2007).

Order: Lecanorales Nannf., Nova Acta R. Soc. Scient. Upsal, ser. 4 8(2): 68 (1932).

Exemplar genera: Cladonia Hill. ex P. Browne 1756, Lecanora Ach. 1809, Parmelia Ach. 1803, Ramalina Ach. 1809, Usnea Dill. ex Adans. 1763

Order: Peltigerales Walt. Watson, New Phytologist 28: 9 (1929).

Exemplar genera: Coccocarpia Pers. 1827, Collema F. H. Wigg. 1780, Nephroma Ach. 1810, Pannaria Del. ex Bory 1828, Peltigera Willd. 1787.
Order: Teloschistales D. Hawksw. \& O. E. Erikss., Syst. Ascom. 5: 183 (1986).

Exemplar genera: Caloplaca Th. Fr. 1861, Teloschistes Norman 1853, Xanthoria (Fr.) Th. Fr. 1860.

Subclass: Ostropomycetidae Reeb, Lutzoni \& Cl. Roux, Mol. Phylogen. Evol. 32: 1055 (2004).

Order: Agyriales Clem. \& Shear, Gen. Fungi: 141 (1931).

Exemplar genera: Agyrium Fr. 1822, Placopsis (Nyl.) Linds. 1867, Trapelia M. Choisy 1929, Trapeliopsis Hertel \& Gotth. Schneid. 1980.

Order: Baeomycetales Lumbsch, Huhndorf \& Lutzoni, ord. nov.

MycoBank no.: MB 501291

Ascomycetes lichenisati algas virides thallo continentes. Ascomata elevata vel raro sessilia, disciformia. Excipulum hyalinum vel pigmentatum, annulatum vel cupulatum. Hymenium non-amyloideum. Paraphyses ramosae, septatae. Asci unitunicati, non-amyloidei vel satis infirme amyloidei, octospori. Ascosporae hyalinae, non-septatae vel septatae, halonatae vel non-halonatae.

Typus: Baeomyces Pers. 1794.

Lichen-forming ascomycetes with chlorococcoid photobiont. Ascomata sessile or rarely stalked, disciform. True exciple hyaline or pigmented, annulate or cupulate. Hymenium non-amyloid. Paraphyses moderately to richly branched, septate. Asci unitunicate, non-amyloid or with slightly amyloid tholi, 8-spored. Ascospores hyaline, non-septate or transseptate, halonate or non-halonate.

Baeomycetales was shown to differ from Agyriales by Kauff \& Lutzoni (2002) and this was confirmed by Miądlikowska et al. (2007) and Lumbsch et al. (2007).

Exemplar genera: Ainoa Lumbsch \& I. Schmitt 2001, Baeomyces Pers. 1794, Phyllobaeis Gierl \& Kalb 1993.

Order: Ostropales Nannf., Nova Acta R. Soc. Scient. Upsal., ser. 4 8(2): 68 (1932).

This order includes also taxa formerly classified in separate orders, such as Gomphillales, Graphidales, Gyalectales and Trichotheliales.

Exemplar genera: Ostropa Fr. 1825, Stictis Pers. 1799, Gyalecta Ach. 1808, Gomphillus Nyl. 1855, Graphis Adans. 1763., Odontotrema Nyl. 1858, Porina Müll. Arg. 1883, Thelotrema Ach. 1803.

Order: Pertusariales M. Choisy ex D. Hawksw. \& O. E. Erikss., Syst. Ascom. 5: 181 (1986).

Synonym: Pertusariales M. Choisy, Bull. mens. Soc. linn. Lyon 18: 12 (1949), nomen invalidum.

This order may not be monophyletic as currently circumscribed, with Ochrolechiaceae and some groups of the heterogeneous Pertusaria clustering in a separate clade, but without support. Nonetheless, a cluster of taxa in a 'core' group of Pertusariales has been strongly supported as monophyletic in phylogenetic analyses by Miądlikowska et al. (2007), Lücking 
et al. (2004), Schmitt et al. (2005), Lutzoni et al. (2004), and Grube et al. (2004).

Exemplar genera: Coccotrema Müll. Arg. 1888, Icmadophila Trevis. 1853, Ochrolechia A. Massal. 1852, Pertusaria DC. 1805.

Lecanoromycetes incertae sedis (not placed in any subclass):

Order: Candelariales Miadl., Lutzoni \& Lumbsch, ord. nov. MycoBank no.: MB 501292

Ascomycetes lichenisati algas virides thallo continentes. Ascomata sessilia, disciformia. Excipulum hyalinum, annulatum. Hymenium amyloideum. Paraphyses ramosae, septatae. Asci unitunicati, amyloidei, ad typum Candelariae dictum pertinentes, octo- vel saepe multispori. Ascosporae hyalinae, non-septatae vel raro 1-septatae.

Typus: Candelaria A. Massal. 1853.

Lichen-forming ascomycetes with chlorococcoid photobiont, predominantly nitrophilous. Thallus of various morphology, yellow to orange (pulvinic acid derivatives). Ascomata apothecial, sessile, with or without a distinct margin, yellow to orange. The ascomatal wall formed from densely septate twisted hyphae. paraphyses mostly simple. Excipulum hyaline, hymenium amyloid. Asci unitunicate of Candelariatype with the amyloid lower part of the apical dome and broad apical cushion, often multispored. Ascospores hyaline, aseptate, rarely 1-septate.

Candelariales was shown to differ from Lecanorales by Wedin et al. (2005) and this was confirmed by Hofstetter et al. (2007) and Miądlikowska et al. (2007).

Exemplar genera: Candelaria A. Massal. 1853, Candelariella Müll. Arg. 1894.

Order: Umbilicariales Lumbsch, Hestmark \& Lutzoni, ord. nov. MycoBank no.: MB 501293

Ascomycetes lichenisati algas virides thallo continentes. Ascomata sessilia, raro immersa usque ad pauce elevata, plerumque atra, irregularia, disciformia. Excipulum pigmentatum, annulatum. Hymenium amyloideum. Paraphyses simplices vel paulum ramosae, septatae. Asci unitunicati, tholo inconspicue amyloideo, 1-8-spori. Ascosporae hyalinae vel brunneae, non-septatae usque ad muriformes.

Typus: Umbilicaria Hoffm. 1789.

Lichen-forming ascomycetes with chlorococcoid photobiont. Ascomata sessile, or rarely immersed or stalked, mostly black, irregular, disciform. True exciple pigmented, annulate. Hymenium amyloid. Paraphyses simple or slightly branched, septate, apically thickened. Asci unitunicate, with slightly amyloid tholi, 1-8-spored. Ascospores hyaline or brown, non-septate to muriform.

Exemplar genera: Lasallia Mérat 1821, Umbilicaria Hoffm. 1789.

Class: Leotiomycetes O. E. Erikss. \& Winka, Myconet 1: 7 (1997). Excluding Geoglossaceae (Wang et al. 2006).

Order: Cyttariales Luttr. ex Gamundí, Darwiniana 16: 502 (1971).
Synonym: Cyttariales Luttr., Univ. Miss. Stud. 24(2): 109 (1951), nomen invalidum.

Exemplar genus: Cyttaria Berk. 1842.

Order: Erysiphales H. Gwynne-Vaughan, Fungi, Ascom., Ustilag., Ured.: 78 (1922).

Exemplar genera: Erysiphe R. Hedw. ex DC. 1805, Blumeria Golovin ex Speer 1975, Uncinula Lév. 1851.

Order: Helotiales Nannf., Nova Acta R. Soc. Scient. Upsal., ser. 4 8(2): 68 (1932).

Based on current character and taxon sampling (Wang et al. 2006, 2007; Spatafora et al. 2007), the monophyly of Helotiales s. lat. is not well supported. There exists a minimum of five helotialean lineages that are intermixed with other leotiomycetan taxa (e.g. Cyttariales, Erysiphales) resulting in a paraphyletic Helotiales s. lat. The interrelationships of these taxa are poorly resolved, however, thus preventing the synthesis of an accurate phylogenetic classification at this time. Leotiomycetes represents one of the more undersampled higher taxa among the Ascomycota, and it is likely that future sampling will result in a phylogenetic classification of a more restricted Helotiales and the recognition of additional orders based on current helotialean families (e.g. Leotiaceae or Helotiaceae, Sclerotiniaceae).

Exemplar genera: Mitrula Fr. 1821, Hymenoscyphus Gray 1821, Ascocoryne J.W. Groves \& D.E. Wilson 1967.

Order: Rhytismatales M. E. Barr ex Minter, in Hawksworth \& Eriksson, Syst. Ascom. 5: 182 (1986).

Synonym: Rhytismatales M. E. Barr, Mem. N. Y. Bot.Gdn 28: 6 (1976), nomen invalidum.

Exemplar genera: Rhytisma Fr. 1818, Lophodermium Chevall. 1826, Cudonia Fr. 1849.

Order: Thelebolales P. F. Cannon, in Kirk et al., Ainsworth \& Bisby's Dict. Fungi (9th edn): xi (2001).

Exemplar genera: Thelebolus Tode 1790, Coprotus Korf ex Korf \& Kimbr. 1967, Ascozonus (Renny) E.C. Hansen 1876.

Class: Lichinomycetes Reeb, Lutzoni \& Cl. Roux., Mol. Phylogen. Evol. 32: 1055 (2004).

Order: Lichinales Henssen \& Büdel, in Hawksworth \& Eriksson, Syst. Ascom. 5: 138 (1986).

Exemplar genera: Heppia Nägeli ex A. Massal. 1854, Lichina C. Agardh 1817, Peltula Nyl. 1853.

Class: Orbiliomycetes O. E. Erikss. \& Baral, in Eriksson et al., Myconet 9: 96 (2003).

Order: Orbiliales Baral, O. E. Erikss., G. Marson \& E. Weber, in Eriksson et al., Myconet 9: 96 (2003).

Exemplar genera: Orbilia Fr. 1849, Hyalorbilia Baral \& G. Marson 2000 .

Class: Pezizomycetes O. E. Erikss. \& Winka, Myconet 1: 8 (1997). 
Order: Pezizales J. Schröt., in Engler \& Prantl (eds), Nat. Pflanzenfam. 1: 173 (1894), as 'Pezizineae'.

Exemplar genera: Peziza Fr. 1822, Glaziella Berk. 1880, Morchella Dill. ex Pers. 1794, Pyronema Carus 1835, Tuber F.H. Wigg. 1780.

Glaziella has been described several times, inter alia as a zygomycete. Gibson et al. (1986) demonstrated it was an ascomycete and proposed a new family and order close to Pezizales, but small subunit rRNA gene sequences show that it should be included in Pezizales (Landvik \& Eriksson 1994).

Class: Sordariomycetes O. E. Erikss. \& Winka, Myconet 1: 10 (1997).

Subclass: Hypocreomycetidae O. E. Erikss. \& Winka, Myconet 1: 6 (1997).

Order: Coronophorales Nannf., Nova Acta R. Soc. Scient. Upsal, ser. 4 8: 54 (1932).

Exemplar genera: Nitschkia G.H. Otth ex P. Karst. 1873, Scortechinia Sacc. 1885, Bertia De Not. 1844, Chaetosphaerella E. Müll. \& C. Booth 1972.

Order: Hypocreales Lindau, in Engler \& Prantl (eds), Nat. Pflanzenfam. 1: 343 (1897).

Exemplar genera: Hypocrea Fr. 1825, Nectria (Fr.) Fr. 1849, Cordyceps (Fr.) Link 1833, Claviceps Tul. 1853, Niesslia Auersw. 1869.

Order: Melanosporales N. Zhang \& M. Blackw., ord. nov. MycoBank no.: MB 501294

Ascomata perithecialia vel nonnumquam ostiolo carentia; peridium ascomatis e basi glomeris ascogonialis oriundum, translucidum; centrum pseudoparenchymaticum, paraphysibus absentibus; asci unitunicati, evanescentes; ascosporae fuscae, poro germinationis utrinque praeditae; anamorphae hyphomycetales. Fungi saepe mycoparasitici.

Typus: Melanospora Corda 1837.

Ascoma perithecial or secondarily cleistothecial, peridium derived from base of an ascogonial coil, translucent; centrum pseudoparenchymatous, paraphyses absent in development; asci unitunicate, evanescent; ascospores dark, with germ pores at both ends; anamorphs hyphomycetous; often mycoparasitic. Exemplar genus: Melanospora Corda 1837.

Order: Microascales Luttr. ex Benny \& Kimbr., Mycotaxon 12: 40 (1980).

Synonym: Microascales Luttr., Univ. Miss. Stud. 24(2): 108 (1951), nomen invalidum.

The group as recognized here includes members of the Halosphaeriales. In Zhang et al. (2007) and Tang et al. (2007), the Halosphaeriales were maintained separate from the Microascales.

Exemplar genera: Microascus Zukal 1885, Petriella Curzi 1930, Halosphaeria Linder 1944, Lignincola Höhnk 1955, Nimbospora J. Koch 1982.
Subclass: Sordariomycetidae O. E. Erikss. \& Winka, Myconet 1: 10 (1997).

Order: Boliniales P. F. Cannon, in Kirk et al., Ainsworth \& Bisby's Dict. Fungi (9th edn): x (2001).

Exemplar genera: Camarops P. Karst. 1873, Apiocamarops Samuels \& J. D. Rogers 1987.

Order: Calosphaeriales M. E. Barr, Mycologia 75: 11 (1983).

This order has not been placed in a subclass but the work of Réblová et al. (2004) shows that it may be related to the Diaporthales. Members of this group were not included in Zhang et al. (2007) or Tang et al. (2007).

Exemplar genera: Calosphaeria Tul. \& C. Tul. 1863, Togniniella Réblová, L. Mostert, W. Gams \& Crous 2004, Pleurostoma Tul. \& C. Tul. 1863.

Order: Chaetosphaeriales Huhndorf, A. N. Mill. \& F. A. Fernández, Mycologia 96: 378 (2004).

Exemplar genera: Chaetosphaeria Tul. \& C. Tul. 1863, Melanochaeta E. Müll., Harr \& Sulmont 1969, Zignoëlla Sacc. 1878, Striatosphaeria Samuels \& E. Müll. 1979.

Order: Coniochaetales Huhndorf, A. N. Mill. \& F. A. Fernández, Mycologia 96: 378 (2004a).

Exemplar genera: Coniochaeta (Sacc.) Cooke 1887, Coniochaetidium Malloch \& Cain 1971.

Order: Diaporthales Nannf., Nova Acta R. Soc. Scient. upsal., ser. 4 8: 53 (1932).

Exemplar genera: Diaporthe Nitschke 1870, Gnomonia Ces. \& De Not. 1863, Cryphonectria (Sacc.) Sacc. \& D. Sacc. 1905, Valsa Fr. 1849.

Order: Ophiostomatales Benny \& Kimbr., Mycotaxon 12: 48 (1980).

Exemplar genera: Ophiostoma Syd. \& P. Syd. 1919, Fragosphaeria Shear 1923.

Order: Sordariales Chadef. ex D. Hawksw. \& O. E. Erikss., Syst. Ascom. 5: 182 (1986).

Synonym: Sordariales Chadef., in Chadefaud \& Emberger, Traité Bot. 1: 594 (1960), nomen invalidum.

Exemplar genera: Sordaria Ces. \& De Not. 1863, Podospora Ces. 1856, Neurospora Shear \& B.O. Dodge 1927, Lasiosphaeria Ces. \& De Not. 1863, Chaetomium Kunze 1817.

Subclass: Xylariomycetidae O. E. Erikss. \& Winka, Myconet 1: 12 (1997).

Order: Xylariales Nannf., Nova Acta R. Soc. Scient. Upsal., ser. 4, 8: 66 (1932).

Exemplar genera: Xylaria Hill ex Schrank 1789, Hypoxylon Bull. 1791, Anthostomella Sacc. 1875, Diatrype Fr. 1849, Graphostroma Piroz. 1974.

Sordariomycetes incertae sedis (not placed in any subclass) 
Order: Lulworthiales Kohlm., Spatafora \& Volkm-Kohlm., Mycologia 92: 456 (2000).

This order includes members formerly placed in the Spathulosporales.

Exemplar genera: Lulworthia G. K. Sutherl. 1916, Lindra I.M. Wilson 1956.

Order: Meliolales Gäum. ex D. Hawksw. \& O. E. Erikss., Syst. Ascom. 5: 180 (1986).

Synonym: Meliolales Gäum., Pilze (2nd edn): 158 (1964), nomen invalidum.

Exemplar genus: Meliola Fr. 1825.

Order: Phyllachorales M. E. Barr, Mycologia 75: 10 (1983). Exemplar genus: Phyllachora Nitschke ex Fuckel 1870.

Order: Trichosphaeriales M. E. Barr, Mycologia 75: 11 (1983). Exemplar genus: Trichosphaeria Fuckel 1870.

Pezizomycotina incertae sedis (not placed in any class)

Order: Lahmiales O. E. Erikss., Mycotaxon 27: 357 (1986). Exemplar genus: Lahmia Körb. 1861.

Order: Medeolariales Korf, in Eriksson Mycotaxon 15: 232 (1982). Exemplar genus: Medeolaria Thaxt. 1922.

Order: Triblidiales O. E. Erikss., Syst. Ascom. 11: 9 (1992). Exemplar genera: Huangshania O. E. Erikss. 1992, Pseudographis Nyl. 1855, Triblidium Rebent. 1804.

Phylum: Basidiomycota R. T. Moore, Bot. Mar. 23: 371 (1980).

Synonyms: Basidiomycota Bold, Morph. Pl.: 7, 198 (1958), nomen invalidum;

Basidiomycetes Whittaker (1959: 220), nomen invalidum. (Table 3, Fig 3) As in the case of Fungi, Moore (1980) validated a name that had already been used by Bold (1957), but he did not cite Bold's work.

Subphylum: Pucciniomycotina R. Bauer, Begerow, J. P. Samp., M. Weiß \& Oberw., Mycol. Progr. 5: 45 (2006).

Equivalent to Urediniomycetes (Kirk et al. 2001; Swann \& Taylor 1995; Swann et al. 2001). The classification of Pucciniomycotina employed here parallels that of Bauer et al. (2006) and Aime et al. (2007).

Class: Pucciniomycetes R. Bauer, Begerow, J. P. Samp., M. Weiß \& Oberw., Mycol. Progr. 5: 48 (2006).

Equivalent to Urediniomycetidae (Swann et al. 2001).

Order: Septobasidiales Couch ex Donk, Persoonia 3: 243 (1964).

Synonym: Septobasidiales Couch, Gen. Septobasidium: 65 (1938), nomen invalidum.

Exemplar genera: Septobasidium Pat. 1892, Auriculoscypha D. A. Reid \& Manim. 1985.

Order: Pachnocybales R. Bauer, Begerow, J. P. Samp., M. Weiß \& Oberw., Mycol. Progr. 5: 48 (2006).
Exemplar genus: Pachnocybe Berk. 1836.

Order: Helicobasidiales R. Bauer, Begerow, J. P. Samp., M. Weiß \& Oberw., Mycol. Progr. 5: 48 (2006).

Exemplar genera: Helicobasidium Pat. 1885, Tuberculina Tode ex Sacc. 1880.

Order: Platygloeales R. T. Moore, Mycotaxon 39: 247 (1990). Equivalent to Platygloeales s. str. (Swann et al. 2001).

Exemplar genera: Platygloea J. Schröt. 1887 s. str., Eocronartium G.F. Atk. 1902.

Order: Pucciniales Clem. \& Shear, Gen. Fungi (2nd edn): 147 (1931).

Equivalent to Uredinales.

Exemplar genera: Puccinia Pers. 1801, Uromyces (Link) Unger 1832.

Class: Cystobasidiomycetes R. Bauer, Begerow, J. P. Samp., M. Weiß \& Oberw., Mycol. Progr. 5: 46 (2006).

Equivalent to the Erythrobasidium-Naohidea-Sakaguchia clade (Swann et al. 2001) and Cystobasidiaceae lineage (Weiß et al. 2004a). Genera of Cystobasidiomycetes that are not placed in any order include Sakaguchia Y. Yamada, K. Maeda \& Mikata 1994, and Cyrenella Goch. 1981 (Aime et al. 2007; Bauer et al. 2006).

Order: Cystobasidiales R. Bauer, Begerow, J. P. Samp., M. Weiß \& Oberw., Mycol. Progr. 5: 46 (2006).

Exemplar genera: Cystobasidium (Lagerh.) Neuhoff 1924, Occultifur Oberw. 1990, Rhodotorula F.C. Harrison 1927 pro parte.

Order: Erythrobasidiales R. Bauer, Begerow, J. P. Samp., M. Weiß \& Oberw., Mycol. Progr. 5: 46 (2006).

Exemplar genera: Erythrobasidium Hamam. Sugiyama \& Komag. 1988, Rhodotorula F. C. Harrison 1927 pro parte, Sporobolomyces Kluyver \& C. B. Niel 1924 pro parte, Bannoa Hamam. 2002.

Order: Naohideales R. Bauer, Begerow, J. P. Samp., M. Weiß \& Oberw., Mycol. Progr. 5: 46 (2006).

Exemplar genus: Naohidea Oberw. 1990.

Class: Agaricostilbomycetes R. Bauer, Begerow, J. P. Samp., M. Weiß \& Oberw., Mycol. Progr. 5: 45 (2006).

Equivalent to Agaricostilbomycetidae (Swann et al. 2001; Weiß et al. 2004a).

Order: Agaricostilbales Oberw. \& R. Bauer, Sydowia 41: 240 (1989).

Exemplar genera: Agaricostilbum J. E. Wright 1970 (emend. Wright, Bandoni \& Oberw. 1981), Chionosphaera D. E. Cox 1976, Kondoa Y. Yamada, Nakagawa \& I. Banno 1989 (emend. Fonseca, Sampaio, Inácio \& Fell 2000).

Order: Spiculogloeales R. Bauer, Begerow, J. P. Samp., M. Weiß \& Oberw., Mycol. Progr. 5: 45 (2006).

Equivalent to Mycogloea group (Weiß et al. 2004a).

Exemplar genera: Mycogloea L. S. Olive 1950, Spiculogloea P. Roberts 1996, Sporobolomyces Kluyver \& C. B. Niel 1924 pro parte. 
Table 3 - Support for major groups of Fungi in selected phylogenetic studies: Basidiomycota

\begin{tabular}{|c|c|c|c|c|c|}
\hline Rank & Taxon & Reference & Data & OTUs & Support \\
\hline Phylum & BASIDIOMYCOTA & James et al. (2006) & LSU, SSU, 5.8S, rpb1, rpb2, tef1 & 50 & $\begin{array}{l}\mathrm{BPP}=1 \\
\mathrm{MLBS}=80\end{array}$ \\
\hline \multirow[t]{2}{*}{ Subphylum } & Pucciniomycotina & Matheny et al. (2007a, fig. 4) & SSU, LSU, 5.8S, rpb1, rpb2, tef1 & 17 & $\begin{array}{l}\mathrm{BPP}=1 \\
\mathrm{MPBS}=100\end{array}$ \\
\hline & & Aime et al. (2007, fig. 2) & LSU, SSU & 109 & $\begin{array}{l}\mathrm{BPP}=1 \\
\mathrm{MPBS}=100\end{array}$ \\
\hline \multirow[t]{4}{*}{ Class } & Pucciniomycetes & Matheny et al. (2007a, fig. 4) & LSU, SSU, 5.8S, rpb1, rpb2, tef1 & 7 & $\begin{array}{l}\mathrm{BPP}>0.95 \\
\mathrm{MPBS}>70\end{array}$ \\
\hline & & Matheny et al. (2007a, fig. 5) & LSU, SSU, 5.8S & 24 & $\begin{array}{l}\mathrm{BPP}=0.97 \\
\mathrm{MPBS} \geq 70\end{array}$ \\
\hline & & Aime et al. (2007, fig. 2) & LSU, SSU & 19 & $\begin{array}{l}\mathrm{BPP}=1 \\
\mathrm{MPBS}=100\end{array}$ \\
\hline & & Aime et al. (2007, fig. 3) & LSU, SSU & 41 & MPBS $=86$ \\
\hline Order & Septobasidiales & Arun Kumar et al. (2007, fig. 7) & LSU, SSU & 4 & $\begin{array}{l}\mathrm{BPP}=1 \\
\mathrm{MPBS}=100\end{array}$ \\
\hline \multirow[t]{2}{*}{ Order } & Pachnocybales & Bauer et al. (2006, fig. 1) & LSU & 1 & NA \\
\hline & & Berres et al. (1995, fig. 4) & LSU & 1 & NA \\
\hline \multirow[t]{2}{*}{ Order } & Helicobasidiales & Aime et al. (2007, fig. 2) & LSU, SSU & 2 & $\begin{array}{l}\mathrm{BPP}=1 \\
\mathrm{MPBS}=96 \\
\mathrm{NJBS}=98\end{array}$ \\
\hline & & Aime et al. (2007, fig. 3) & LSU, SSU & 10 & $\mathrm{MPBS}=87$ \\
\hline \multirow[t]{3}{*}{ Order } & Platygloeales & Aime et al. (2007, fig. 2) & LSU, SSU & 4 & $\begin{array}{l}\mathrm{BPP}=1 \\
\mathrm{MPBS}=100 \\
\mathrm{NJBS}=100\end{array}$ \\
\hline & & Aime et al. (2007, fig. 3) & LSU, SSU & 8 & MPBS $=99$ \\
\hline & & Matheny et al. (2007a, fig. 4) & LSU, SSU, 5.8S, rpb1, rpb2, tef1 & 2 & $\begin{array}{l}\mathrm{BPP}>0.95 \\
\mathrm{MPBS}>70\end{array}$ \\
\hline \multirow[t]{4}{*}{ Order } & Pucciniales & Aime et al. (2007, fig. 2) & LSU, SSU & 12 & $\begin{array}{l}\mathrm{BPP}=1 \\
\mathrm{MPBS}=100 \\
\mathrm{NJBS}=100\end{array}$ \\
\hline & & Aime (2006) & LSU & 46 & MPBS $=99$ \\
\hline & & Wingfield et al. (2004) & SSU & 72 & MPBS $<50$ \\
\hline & & Matheny et al. (2007a, fig. 4) & LSU, SSU, 5.8S, rpb1, rpb2, tef1 & 5 & $\begin{array}{l}\mathrm{BPP}>0.95 \\
\mathrm{MPBS}>70\end{array}$ \\
\hline \multirow[t]{3}{*}{ Class } & Cystobasidiomycetes & Aime et al. (2007, fig. 2) & LSU, SSU & 27 & $\begin{array}{l}\mathrm{BPP}=1 \\
\mathrm{MPBS}=100 \\
\mathrm{NJBS}=96\end{array}$ \\
\hline & & Sampaio (2004, fig. 1) & LSU & 11 & $\mathrm{BPP}=0.92$ \\
\hline & & Sampaio (2004, fig. 2) & LSU & 26 & $\mathrm{BPP}=0.98$ \\
\hline \multirow[t]{3}{*}{ Order } & Cystobasidiales & Nagahama et al. (2006, fig. 2) & LSU, SSU, 5.8S, tef1 & 9 & MLBS $=100$ \\
\hline & & Aime et al. (2007, fig. 2) & LSU, SSU & 12 & $\begin{array}{l}\mathrm{BPP}=1 \\
\mathrm{MPBS}=100 \\
\mathrm{NJBS}=100\end{array}$ \\
\hline & & Sampaio (2004, fig. 2) & LSU & 8 & $\mathrm{BPP}=1$ \\
\hline \multirow[t]{3}{*}{ Order } & Erythrobasidiales & Nagahama et al. (2006, fig. 2) & LSU, SSU, 5.8S, tef1 & 21 & MLBS $=72$ \\
\hline & & Aime et al. (2007, fig. 2) & LSU, SSU & 14 & $\begin{array}{l}\mathrm{BPP}=1 \\
\mathrm{MPBS}=83 \\
\mathrm{NJBS}=91\end{array}$ \\
\hline & & Sampaio (2004, fig. 2) & LSU & 18 & $\mathrm{BPP}=1$ \\
\hline \multirow[t]{2}{*}{ Order } & Naohideales & Aime et al. $(2007$, fig. 3$)$ & LSU, SSU & 2 & $\mathrm{MPBS}=98$ \\
\hline & & Weiß et al. (2004) & LSU & 3 & $\begin{array}{l}\mathrm{BPP}=0.94 \\
\mathrm{NJBS}<50\end{array}$ \\
\hline \multirow[t]{3}{*}{ Class } & Agaricostilbomycetes & Aime et al. (2007, fig. 2) & LSU, SSU & 25 & $\begin{array}{l}\mathrm{BPP}=1 \\
\mathrm{MPBS}<70 \\
\mathrm{NJBS}<70\end{array}$ \\
\hline & & Bauer et al. (2006, fig. 2) & LSU, SSU & 4 & $\mathrm{NJBS}=89$ \\
\hline & & Matheny et al. (2007a, fig. 5) & LSU, SSU, $5.8 \mathrm{~S}$ & 8 & $\begin{array}{l}\mathrm{BPP}=1 \\
\mathrm{MPBS}>70\end{array}$ \\
\hline \multirow[t]{4}{*}{ Order } & Agaricostilbales & Aime et al. (2007, fig. 2) & LSU, SSU & 22 & $\begin{array}{l}\mathrm{BPP}=1 \\
\mathrm{MPBS}=100 \\
\mathrm{NJBS}=100\end{array}$ \\
\hline & & Aime et al. (2007, fig. 2) & LSU, SSU & 34 & $\mathrm{MPBS}=98$ \\
\hline & & Sampaio (2004, fig. 1) & LSU & 7 & $\mathrm{BPP}=1$ \\
\hline & & Sampaio (2004, fig. 2) & LSU & 23 & $\mathrm{BPP}=1$ \\
\hline
\end{tabular}




\begin{tabular}{|c|c|c|c|c|c|}
\hline Rank & Taxon & Reference & Data & OTUs & Support \\
\hline & & Fell et al. (2001) & LSU & 24 & $\mathrm{MPBS}=64$ \\
\hline \multirow[t]{5}{*}{ Order } & Spiculogloeales & Aime et al. (2007, fig. 2) & LSU, SSU & 3 & $\mathrm{BPP}=1$ \\
\hline & & & & & $\mathrm{MPBS}=100$ \\
\hline & & & & & NJBS $=100$ \\
\hline & & Aime et al. (2007, fig. 3) & LSU, SSU & 7 & MPBS $=74$ \\
\hline & & Bauer et al. (2006, fig. 2) & LSU, SSU & 2 & $\mathrm{NJBS}=90$ \\
\hline \multirow[t]{8}{*}{ Class } & Microbotryomycetes & Aime et al. (2007, fig. 2) & LSU, SSU & 31 & $\mathrm{BPP}=1$ \\
\hline & & & & & MPBS $=100$ \\
\hline & & & & & $\mathrm{NJBS}=100$ \\
\hline & & Aime et al. (2007, fig. 3) & LSU, SSU & 60 & MPBS $=74$ \\
\hline & & Matheny et al. (2007a, fig. 4) & LSU, SSU, 5.8S, & 6 & $\mathrm{BPP}>0.95$ \\
\hline & & & $r p b 1, r p b 2$, tef1 & & MPBS $>70$ \\
\hline & & Sampaio (2004, fig. 2) & LSU & $49^{*}$ & $\mathrm{BPP}=0.87$ \\
\hline & & Fell et al. (2001) & LSU & 78 & $\mathrm{MPBS}=75$ \\
\hline Order & Heterogastridiales & Bauer et al. (2006, fig. 2) & LSU, SSU & 1 & NA \\
\hline \multirow[t]{4}{*}{ Order } & Microbotryales & Aime et al. (2007, fig. 2) & LSU, SSU & 4 & $\mathrm{BPP}=1$ \\
\hline & & & & & $\mathrm{MPBS}=99$ \\
\hline & & & & & $\mathrm{NJBS}=94$ \\
\hline & & Aime et al. (2007, fig. 3) & LSU, SSU & 12 & $\mathrm{MPBS}=82$ \\
\hline \multirow[t]{4}{*}{ Order } & Leucosporidiales & Aime et al. (2007, fig. 2) & LSU, SSU & 3 & $\mathrm{BPP}=0.98$ \\
\hline & & & & & $\mathrm{MPBS}=85$ \\
\hline & & & & & $\mathrm{NJBS}=100$ \\
\hline & & Aime et al. (2007, fig. 3) & LSU, SSU & 9 & MPBS $=67$ \\
\hline \multirow[t]{5}{*}{ Order } & Sporidiobolales & Aime et al. (2007, fig. 2) & LSU, SSU & 13 & $\mathrm{BPP}=1$ \\
\hline & & & & & $\mathrm{MPBS}=74$ \\
\hline & & & & & $\mathrm{NJBS}=68$ \\
\hline & & Aime et al. (2007, fig. 3) & LSU, SSU & 17 & MPBS $=69$ \\
\hline & & Sampaio (2004, fig. 2) & LSU & 20 & $\mathrm{BPP}=0.98$ \\
\hline \multirow[t]{5}{*}{ Class/Order } & Atractiellomycetes, & Aime et al. (2007, fig. 2) & LSU, SSU & 4 & $\mathrm{BPP}=1$ \\
\hline & Atractiellales & & & & MPBS $=80$ \\
\hline & & & & & $\mathrm{NJBS}=96$ \\
\hline & & Aime et al. (2007, fig. 3) & LSU, SSU & 8 & MPBS $=68$ \\
\hline & & Bauer et al. (2006, fig. 2) & LSU, SSU & 7 & $\mathrm{NJBS}=68$ \\
\hline \multirow[t]{5}{*}{ Class/Order } & Classiculomycetes, & Aime et al. (2007, fig. 2) & LSU, SSU & 2 & $\mathrm{BPP}=1$ \\
\hline & Classiculales & & & & $\mathrm{MPBS}=100$ \\
\hline & & & & & $\mathrm{NJBS}=100$ \\
\hline & & Weiß et al. (2004, figs. 1-2) & LSU & 2 & $\mathrm{BPP}=1$ \\
\hline & & & & & $\mathrm{NJBS}=99$ \\
\hline \multirow[t]{2}{*}{ Class/Order } & Mixiomycetes, Mixiales & Aime et al. (2007, fig. 2) & LSU, SSU & 1 & NA \\
\hline & & Bauer et al. (2006, fig. 2) & LSU, SSU & 1 & NA \\
\hline \multirow[t]{2}{*}{ Class/Order } & $\begin{array}{l}\text { Cryptomycocolacomycetes, } \\
\text { Cryptomycocolacales }\end{array}$ & Aime et al. (2007, fig. 3) & LSU, SSU & 1 & NA \\
\hline & & Bauer et al. (2006, fig. 1) & LSU & 2 & $\mathrm{NJBS}=100$ \\
\hline \multirow[t]{4}{*}{ Subphylum } & Ustilaginomycotina & Matheny et al. (2007a, fig. 4) & LSU, SSU, 5.8S, & 24 & $\mathrm{BPP}=1$ \\
\hline & & & rpb1, rpb2, tef1 & & $\mathrm{MPBS}=100$ \\
\hline & & Matheny et al. (2007a, fig. 5) & LSU, SSU, $5.8 \mathrm{~S}$ & 59 & $\mathrm{BPP}=1$ \\
\hline & & & & & MPBS $>70$ \\
\hline \multirow[t]{11}{*}{ Class } & Ustilaginomycetes & Bauer et al. (2006, fig. 2) & LSU, SSU & 21 & $\mathrm{NJBS}=100$ \\
\hline & & Matheny et al. (2007a, fig. 4) & LSU, SSU, 5.8S, & 12 & $\mathrm{BPP}>0.95$ \\
\hline & & & $r p b 1, r p b 2$, tef1 & & MPBS $>70$ \\
\hline & & Matheny et al. (2007a, fig. 5) & LSU, SSU, 5.8S & 25 & $\mathrm{BPP}=1$ \\
\hline & & & & & MPBS $>70$ \\
\hline & & Begerow et al. (2007, fig. 1) & LSU, ITS, atp6, $\beta$ tub & 53 & $\mathrm{BPP}=1$ \\
\hline & & & & & $\mathrm{MPBS}=83$ \\
\hline & & & & & $\mathrm{NJBS}=77$ \\
\hline & & Bauer et al. (2001, figs. 33-34) & LSU & 36 & MPBS $=79$ \\
\hline & & & & & $\mathrm{NJBS}=93$ \\
\hline & & Fell et al. (2001, fig. 24) & LSU & 27 & $\mathrm{NJBS}=86$ \\
\hline \multirow[t]{6}{*}{ Order } & Urocystales & Begerow et al. (2007, fig. 1) & LSU, ITS, atp6, $\beta$ tub & 5 & $\mathrm{BPP}=1$ \\
\hline & & & & & MPBS $=66$ \\
\hline & & & & & $\mathrm{NJBS}=96$ \\
\hline & & Matheny et al. (2007a, fig. 4) & LSU, SSU, 5.8S, rpb1, rpb2, tef1 & 1 & NA \\
\hline & & Bauer et al. (2001, figs. 33-34) & LSU & 9 & MPBS $=95^{3}$ \\
\hline & & & & & $\mathrm{NJBS}=96^{3}$ \\
\hline
\end{tabular}




\begin{tabular}{|c|c|c|c|c|c|}
\hline Rank & Taxon & Reference & Data & OTUs & Support \\
\hline \multirow[t]{3}{*}{ Order } & Ustilaginales & Matheny et al. (2007a, fig. 4) & LSU, SSU, 5.8S, rpb1, rpb2, tef1 & 10 & $\begin{array}{l}\mathrm{BPP}>0.95 \\
\mathrm{MPBS}>70\end{array}$ \\
\hline & & Matheny et al. (2007a, fig. 5) & LSU, SSU, $5.8 \mathrm{~S}$ & 23 & $\begin{array}{l}\mathrm{BPP}>0.95 \\
\mathrm{MPBS}>70\end{array}$ \\
\hline & & Begerow et al. (2007, fig. 1) & LSU, ITS, atp6, $\beta$ tub & 46 & $\begin{array}{l}\mathrm{BPP}=1 \\
\mathrm{MPBS}<60 \\
\mathrm{NJBS}<60\end{array}$ \\
\hline \multirow[t]{3}{*}{ Class } & Exobasidiomycetes & Matheny et al. (2007a, fig. 4) & LSU, SSU, 5.8S, rpb1, rpb2, tef1 & 12 & $\begin{array}{l}\mathrm{BPP}>0.95 \\
\mathrm{MPBS}<50\end{array}$ \\
\hline & & Begerow et al. (2007, fig. 1) & LSU, ITS, atp6, $\beta$ tub & 35 & $\begin{array}{l}\mathrm{BPP}<0.60 \\
\mathrm{MPBS}<60 \\
\mathrm{NJBS}<60\end{array}$ \\
\hline & & Bauer et al. (2001, figs. 33-34) & LSU & 36 & $\begin{array}{l}\text { MPBS }=85 \\
\text { NJBS }=56\end{array}$ \\
\hline \multirow[t]{4}{*}{ Order } & Doassansiales & Matheny et al. (2007a, fig. 4) & LSU, SSU, 5.8S, rpb1, rpb2, tef1 & 1 & NA \\
\hline & & Matheny et al. (2007a, fig. 5) & LSU, SSU, $5.8 \mathrm{~S}$ & 4 & $\begin{array}{l}\mathrm{BPP}>0.95 \\
\mathrm{MPBS}>70\end{array}$ \\
\hline & & Begerow et al. (2007, fig. 1) & LSU, ITS, atp6, $\beta$ tub & 4 & $\begin{array}{l}\mathrm{BPP}=1 \\
\mathrm{MPBS}=84 \\
\mathrm{NJBS}=77\end{array}$ \\
\hline & & Bauer et al. (2001, figs. 33-34) & LSU & 5 & $\begin{array}{l}\mathrm{MPBS}=96 \\
\mathrm{NJBS}=97\end{array}$ \\
\hline \multirow[t]{3}{*}{ Order } & Entylomatales & Matheny et al. (2007a, fig. 4) & LSU, SSU, 5.8S, rpb1, rpb2, tef1 & 4 & $\begin{array}{l}\mathrm{BPP}>0.95 \\
\mathrm{MPBS}>70\end{array}$ \\
\hline & & Begerow et al. (2007, fig. 1) & LSU, ITS, atp6, $\beta$ tub & 3 & $\begin{array}{l}\mathrm{BPP}=1 \\
\mathrm{MPBS}<60 \\
\mathrm{~N} J \mathrm{BS}<60\end{array}$ \\
\hline & & Bauer et al. (2001, figs. 33-34) & LSU & 9 & $\begin{array}{l}\mathrm{MPBS}=72 \\
\mathrm{NJBS}=91\end{array}$ \\
\hline \multirow[t]{3}{*}{ Order } & Exobasidiales & Matheny et al. (2007a, fig. 4) & LSU, SSU, 5.8S, rpb1, rpb2, tef1 & 2 & $\begin{array}{l}\mathrm{BPP}>0.95 \\
\mathrm{MPBS}>70\end{array}$ \\
\hline & & Matheny et al. (2007a, fig. 5) & LSU, SSU & 6 & $\begin{array}{l}\mathrm{BPP}>0.95 \\
\mathrm{MPBS}>70\end{array}$ \\
\hline & & Begerow et al. (2007, fig. 1) & LSU, ITS, atp6, $\beta$ tub & 8 & $\begin{array}{l}\mathrm{BPP}=1 \\
\mathrm{MPBS}<60 \\
\mathrm{NJBS}=61\end{array}$ \\
\hline \multirow[t]{3}{*}{ Order } & Georgefischeriales & Matheny et al. (2007a, fig. 4) & LSU, SSU, 5.8S, rpb1, rpb2, tef1 & 2 & $\begin{array}{l}\mathrm{BPP}>0.95 \\
\mathrm{MPBS}>70\end{array}$ \\
\hline & & Begerow et al. (2007, fig. 1) & LSU, ITS, atp6, $\beta$ tub & 5 & $\begin{array}{l}\mathrm{BPP}<0.60 \\
\mathrm{MPBS}<60 \\
\mathrm{NJBS}<60\end{array}$ \\
\hline & & Bauer et al. (2001, figs. 33-34) & LSU & 9 & $\begin{array}{l}\text { MPBS }=86 \\
\text { NJBS }=65\end{array}$ \\
\hline \multirow[t]{3}{*}{ Order } & Microstromatales & Matheny et al. (2007a, fig. 4) & LSU, SSU, 5.8S, rpb1, rpb2, tef1 & 1 & NA \\
\hline & & Matheny et al. (2007a, fig. 5) & LSU, SSU, $5.8 \mathrm{~S}$ & 3 & $\begin{array}{l}\mathrm{BPP}>0.95 \\
\mathrm{MPBS}>70\end{array}$ \\
\hline & & Begerow et al. (2007, fig. 1) & LSU, ITS, atp6, $\beta$ tub & 5 & $\begin{array}{l}\mathrm{BPP}=1 \\
\mathrm{MPBS}=63 \\
\mathrm{NJBS}=67\end{array}$ \\
\hline \multirow[t]{4}{*}{ Order } & Tilletiales & Matheny et al. (2007a, fig. 4) & LSU, SSU, 5.8S, rpb1, rpb2, tef1 & 2 & $\begin{array}{l}\mathrm{BPP}>0.95 \\
\mathrm{MPBS}>70\end{array}$ \\
\hline & & Matheny et al. (2007a, fig. 5) & LSU, SSU, $5.8 \mathrm{~S}$ & 7 & $\begin{array}{l}\mathrm{BPP}>0.95 \\
\mathrm{MPBS}>70\end{array}$ \\
\hline & & Begerow et al. (2007, fig. 1) & LSU, ITS, atp6, $\beta$ tub & 5 & $\begin{array}{l}\mathrm{BPP}=1 \\
\mathrm{MPBS}=76 \\
\mathrm{NJBS}=64\end{array}$ \\
\hline & $\begin{array}{l}\text { Ustilaginomycotina } \\
\text { incertae sedis (not } \\
\text { placed in any class) }\end{array}$ & & & & \\
\hline \multirow[t]{2}{*}{ Order } & Malasseziales & Matheny et al. (2007a, fig. 4) & LSU, SSU, 5.8S, rpb1, rpb2, tef1 & 1 & NA \\
\hline & & Begerow et al. (2007, fig. 1) & LSU, ITS, atp6, $\beta$ tub & 2 & $\begin{array}{l}\mathrm{BPP}=1 \\
\mathrm{MPBS}=100 \\
\mathrm{NJBS}=100\end{array}$ \\
\hline
\end{tabular}




\begin{tabular}{|c|c|c|c|c|c|}
\hline Rank & Taxon & Reference & Data & OTUs & Support \\
\hline & & Bauer et al. (2001, figs. 33-34) & LSU & 4 & $\begin{array}{l}\text { MPBS }=100 \\
\text { NJBS }=100\end{array}$ \\
\hline Subphylum & Agaricomycotina & Matheny et al. (2007b, fig. 6) & LSU, SSU, 5.8S, rpb2, tef1 & 125 & $\begin{array}{l}\mathrm{BPP}=1 \\
\mathrm{MPBS}=95\end{array}$ \\
\hline \multirow[t]{2}{*}{ Class } & Tremellomycetes & Matheny et al. (2007a, fig. 4) & SSU, LSU, 5.8S, rpb1, rpb2, tef1 & 5 & $\begin{array}{l}\mathrm{BPP}>0.95 \\
\mathrm{MPBS}=50-69\end{array}$ \\
\hline & & Fell et al. (2001, figs. 19, 22) & LSU & 139 & $\mathrm{MPBS}=100$ \\
\hline \multirow[t]{2}{*}{ Order } & Cystofilobasidiales & Matheny et al. (2007a, fig. 5) & LSU, SSU, $5.8 \mathrm{~S}$ & 5 & $\begin{array}{l}\mathrm{BPP}=1 \\
\mathrm{MPBS} \geq 70\end{array}$ \\
\hline & & Fell \& Scorzetti (2004, fig. 1) & LSU & 16 & $\begin{array}{l}\mathrm{BPP}=1 \\
\mathrm{MPBS}=83\end{array}$ \\
\hline Order & Filobasidiales & Fell et al. (2001, figs. 19, 22) & LSU & 34 & MPBS $=96$ \\
\hline \multirow[t]{2}{*}{ Order } & Tremellales & Matheny et al. (2007a, fig. 5) & LSU, SSU, 5.8S & 5 & $\begin{array}{l}\mathrm{BPP} \geq 0.95 \\
\mathrm{MPBS} \geq 70\end{array}$ \\
\hline & & Fell et al. (2001, figs. 19, 22) & LSU & 89 & $\mathrm{MPBS}=56$ \\
\hline \multirow[t]{2}{*}{ Class/Order } & $\begin{array}{l}\text { Dacrymycetes, } \\
\text { Dacrymycetales }\end{array}$ & Matheny et al. (2007b, fig. 6) & LSU, SSU, 5.8S, rpb2, tef1 & 4 & $\begin{array}{l}\mathrm{BPP}=1 \\
\mathrm{MPBS}=100\end{array}$ \\
\hline & & Weiß \& Oberwinkler (2001, fig. 6) & LSU & 9 & $\mathrm{NJBS}=99$ \\
\hline \multirow[t]{2}{*}{ Class } & Agaricomycetes & Matheny et al. (2007b, fig. 6) & LSU, SSU, 5.8S, rpb2, tef1 & 119 & $\begin{array}{l}\mathrm{BPP}=1 \\
\mathrm{MPBS}=95\end{array}$ \\
\hline & & James et al. (2006) & LSU, SSU, 5.8S, rpb1, rpb2, tef1 & 37 & $\begin{array}{l}\mathrm{BPP}=1 \\
\mathrm{MLBS}=92\end{array}$ \\
\hline \multirow[t]{3}{*}{ Subclass } & Agaricomycetidae & Matheny et al. (2007b, fig. 6) & LSU, SSU, 5.8S, rpb2, tef1 & 63 & $\begin{array}{l}\mathrm{BPP}=1 \\
\mathrm{MPBS}=96\end{array}$ \\
\hline & & Binder \& Hibbett (2007, fig. 2) & LSU, SSU, 5.8S, mt-LSU, atp 6 & 47 & $\begin{array}{l}\text { BPP }>0.98 \\
\text { MLBS }=88\end{array}$ \\
\hline & & Binder et al. (2005, fig. 1) & LSU, SSU, mt-LSU, mt-SSU & 46 & $\mathrm{MPBS}=62$ \\
\hline \multirow[t]{4}{*}{ Order } & Agaricales & Matheny et al. (2006, fig. 2) & LSU, SSU, 5.8S & 230 & $\mathrm{BPP}=0.84$ \\
\hline & & Matheny et al. (2006, fig. 3) & LSU, SSU, 5.8S, rpb1, rpb2 & 238 & $\begin{array}{l}\mathrm{BPP}=1 \\
\mathrm{MPBS}=43\end{array}$ \\
\hline & & Matheny et al. (2007b, fig. 6) & LSU, SSU, 5.8S, rpb2, tef1 & 41 & $\begin{array}{l}\mathrm{BPP}=1 \\
\mathrm{MPBS}=76\end{array}$ \\
\hline & & Moncalvo et al. (2002, fig. 2) & LSU & 786 & MPBS $<50$ \\
\hline \multirow[t]{2}{*}{ Order } & Atheliales & Larsson et al. (2004, fig. 1) & LSU & 8 & MPBS $=97$ \\
\hline & & Binder et al. (2005, fig. 4) & LSU, SSU, mt-LSU, mt-SSU & 3 & MPBS $=75$ \\
\hline \multirow[t]{3}{*}{ Order } & Boletales & Matheny et al. (2007b, fig. 6) & LSU, SSU, 5.8S, rpb2, tef1 & 11 & $\begin{array}{l}\mathrm{BPP}=1 \\
\mathrm{MPBS}=100\end{array}$ \\
\hline & & Binder \& Hibbett (2007, fig. 2) & LSU, SSU, 5.8S, mt-LSU, atp6 & 42 & $\begin{array}{l}\mathrm{BPP}>0.98 \\
\mathrm{MLBS}=99\end{array}$ \\
\hline & & Binder \& Hibbett (2007, fig. 3) & LSU & 301 & $\mathrm{BPP}>0.98$ \\
\hline \multirow[t]{2}{*}{ Subclass } & Phallomycetidae & Hosaka et al. (2007, fig. 2) & LSU, mt-SSU, atp6, rpb2, tef1 & 222 & $\begin{array}{l}\mathrm{BPP}=1 \\
\mathrm{MPBS}=98\end{array}$ \\
\hline & & Matheny et al. (2007b, fig. 6) & LSU, SSU, 5.8S, rpb2, tef1 & 3 & $\begin{array}{l}\mathrm{BPP}=1 \\
\mathrm{MPBS}=100\end{array}$ \\
\hline Order & Geastrales & Hosaka et al. (2007, fig. 2) & LSU, mt-SSU, atp6, rpb2, tef1 & 21 & $\begin{array}{l}\mathrm{BPP}=1 \\
\mathrm{MPBS}=59\end{array}$ \\
\hline Order & Gomphales & Hosaka et al. (2007, fig. 2) & LSU, mt-SSU, atp6, rpb2, tef1 & 61 & $\begin{array}{l}\mathrm{BPP}=1 \\
\mathrm{MPBS}=63\end{array}$ \\
\hline Order & Hysterangiales & Hosaka et al. (2007, fig. 2) & LSU, mt-SSU, atp6, rpb2, tef1 & 99 & $\begin{array}{l}\mathrm{BPP}=1 \\
\mathrm{MPBS}=98\end{array}$ \\
\hline \multirow[t]{2}{*}{ Order } & Phallales & Hosaka et al. (2007, fig. 2) & LSU, mt-SSU, atp6, rpb2, tef1 & 41 & $\begin{array}{l}\mathrm{BPP}=1 \\
\mathrm{MPBS}=84\end{array}$ \\
\hline & $\begin{array}{l}\text { Agaricomycetes incertae } \\
\text { sedis (not placed in } \\
\text { any subclass): }\end{array}$ & & & & \\
\hline \multirow[t]{2}{*}{ Order } & Auriculariales & Matheny et al. (2007b, fig. 6) & LSU, SSU, 5.8S, rpb2, tef1 & 3 & $\begin{array}{l}\mathrm{BPP}=1 \\
\mathrm{MPBS}=100\end{array}$ \\
\hline & & Weiß \& Oberwinkler (2001, fig. 6) & LSU & 43 & NJBS $<60$ \\
\hline \multirow[t]{3}{*}{ Order } & Cantharellales & Matheny et al. (2007b, fig. 6) & LSU, SSU, 5.8S, rpb2, tef1 & 11 & $\begin{array}{l}\mathrm{BPP}=1 \\
\mathrm{MPBS}=69\end{array}$ \\
\hline & & Moncalvo et al. (2007, fig. 1) & LSU, SSU, mtSSU, rpb2 & 29 & $\begin{array}{l}\mathrm{BPP}<0.50 \\
\mathrm{MPBS}<50\end{array}$ \\
\hline & & Binder et al. (2005, fig. 4) & LSU, SSU, mt-LSU, mt-SSU & 31 & MPBS $<50$ \\
\hline Order & Corticiales & Larsson et al. (2004, fig. 1) & LSU & 7 & MPBS $=96$ \\
\hline
\end{tabular}




\begin{tabular}{|c|c|c|c|c|c|}
\hline Rank & Taxon & Reference & Data & OTUs & Support \\
\hline & & Binder et al. (2005, fig. 4) & LSU, SSU, mt-LSU, mt-SSU & 8 & $\mathrm{MPBS}=81$ \\
\hline \multirow[t]{5}{*}{ Order } & Gloeophyllales & Thorn et al. (2000, fig. 5) & LSU & 5 & $\mathrm{MPBS}=71$ \\
\hline & & Binder et al. (2005, fig. 4) & LSU, SSU, mt-LSU, mt-SSU & 6 & $\mathrm{MPBS}=54$ \\
\hline & Hymenochaetales & Matheny et al. (2007b, fig. 6) & LSU, SSU, 5.8S, rpb2, tef1 & 7 & $\begin{array}{l}\mathrm{BPP}=1 \\
\mathrm{MPBS}=63\end{array}$ \\
\hline & & Larsson et al. (2007, fig. 3) & LSU, 5.8S & 174 & $\mathrm{BPP}=1$ \\
\hline & & Wagner \& Fischer (2002, fig. 2) & LSU & 104 & $\mathrm{NJBS}=100$ \\
\hline \multirow[t]{2}{*}{ Order } & Polyporales & Matheny et al. (2007b, fig. 6) & LSU, SSU, 5.8S, rpb2, tef1 & 16 & $\begin{array}{l}\mathrm{BPP}=1 \\
\mathrm{MPBS}=85\end{array}$ \\
\hline & & Binder et al. (2005, fig. 4) & LSU, SSU, mt-LSU, mt-SSU & 122 & MPBS $<50$ \\
\hline \multirow[t]{3}{*}{ Order } & Russulales & Matheny et al. (2007b, fig. 6) & LSU, SSU, 5.8S, rpb2, tef1 & 8 & $\begin{array}{l}\mathrm{BPP}=1 \\
\mathrm{MPBS}=99\end{array}$ \\
\hline & & Larsson \& Larsson (2003, fig. 1) & LSU, $5.8 \mathrm{~S}$ & 127 & MPBS $=96$ \\
\hline & & Miller et al. (2007, fig. 2) & LSU, ITS & 143 & $\mathrm{MPBS}=100$ \\
\hline \multirow[t]{2}{*}{ Order } & Sebacinales & Matheny et al. (2007b, fig. 6) & LSU, SSU, 5.8S, rpb2, tef1 & 2 & $\begin{array}{l}\mathrm{BPP}=1 \\
\mathrm{MPBS}=100\end{array}$ \\
\hline & & Weiß \& Oberwinkler (2001, fig. 6) & LSU & 9 & NJBS $=99$ \\
\hline \multirow[t]{3}{*}{ Order } & Thelephorales & Matheny et al. (2007b, fig. 6) & LSU, SSU, 5.8S, rpb2, tef1 & 2 & $\begin{array}{l}\mathrm{BPP}=1 \\
\mathrm{MPBS}=100\end{array}$ \\
\hline & & Binder et al. (2005, fig. 4) & LSU, SSU, mt-LSU, mt-SSU & 13 & $\mathrm{MPBS}=97$ \\
\hline & & Larsson et al. (2004, fig. 1) & LSU, $5.8 \mathrm{~S}$ & 11 & $\mathrm{MPBS}=86$ \\
\hline \multirow[t]{4}{*}{ Order } & Trechisporales & Matheny et al. (2007b, fig. 6) & LSU, SSU, 5.8S, rpb2, tef1 & 2 & $\begin{array}{l}\mathrm{BPP}=1 \\
\mathrm{MPBS}=100\end{array}$ \\
\hline & & Binder et al. (2005, fig. 4) & LSU, SSU, mt-LSU, mt-SSU & 20 & MPBS $=69$ \\
\hline & & Larsson et al. (2004, fig. 1) & LSU, $5.8 \mathrm{~S}$ & 12 & MPBS $=99$ \\
\hline & $\begin{array}{l}\text { Basidiomycota incertae } \\
\text { sedis (not placed in } \\
\text { any subphylum): }\end{array}$ & & & & \\
\hline \multirow[t]{2}{*}{ Class/Order } & $\begin{array}{l}\text { Wallemiomycetes, } \\
\text { Wallemiales }\end{array}$ & Matheny et al. (2007a, fig. 4) & LSU, SSU, 5.8S, rpb1, rpb2, tef1 & 3 & $\begin{array}{l}\mathrm{BPP}>0.95 \\
\mathrm{MPBS}>70\end{array}$ \\
\hline & & Matheny et al. (2007a, fig. 5) & LSU, SSU, $5.8 \mathrm{~S}$ & 3 & $\begin{array}{l}\mathrm{BPP}=1 \\
\mathrm{MPBS}>70\end{array}$ \\
\hline \multirow[t]{2}{*}{ Class/Order } & $\begin{array}{l}\text { Entorrhizomycetes, } \\
\text { Entorrhizales }\end{array}$ & Matheny et al. (2007a, fig. 5) & LSU, SSU, $5.8 \mathrm{~S}$ & 3 & $\begin{array}{l}\mathrm{BPP}=1 \\
\mathrm{MPBS}>70\end{array}$ \\
\hline & & Bauer et al. (2001, figs. 33-34) & LSU & 2 & $\begin{array}{l}\mathrm{MPBS}=100 \\
\mathrm{NJBS}=100\end{array}$ \\
\hline
\end{tabular}

Class: Microbotryomycetes R. Bauer, Begerow, J. P. Samp., M. Weiß \& Oberw., Mycol. Progr. 5: 47 (2006).

Equivalent to Microbotryomycetidae (Swann et al. 2001; Weiß et al. 2004a). The backbone of the Microbotryomycetes remains poorly resolved, and several genera of Microbotryomycetes are not placed in any order, including Colacogloea Oberw. \& R. Bauer 1991, Atractocolax R. Kirschner, R. Bauer \& Oberw. 1999, Krieglsteinera Pouzar 1987, Camptobasidium Marvanová \& Suberkr. 1990, Kriegeria Bres. 1891 and certain species of the polyphyletic genera Sporobolomyces Kluyver \& C. B. Niel 1924 pro parte, Rhodotorula F. C. Harrison 1927 pro parte, and Leucosporidium Fell, Statzell, I. L. Hunter \& Phaff 1970, and others (Aime et al. 2007; Bauer et al. 2006; Sampaio et al. 2003; Weiß et al. 2004a).

Order: Heterogastridiales Oberw., R. Bauer \& Bandoni R. J., Mycologia 82: 57 (1990).

Exemplar genus: Heterogastridium Oberw. \& R. Bauer 1990.

Bauer et al. (2006) placed Colacogloea, Atractocolax and Krieglsteinera in the Heterogastridiales. However, analyses of Bauer et al. (2006) and Aime et al. (2007) suggest that Heterogastridium and Colacogloea do not form a clade, while Atractocolax and Krieglsteinera have yet to be sampled in molecular phylogenetic studies.

Order: Microbotryales R. Bauer \& Oberw., in Bauer et al., Can. J. Bot. 75: 1309 (1997).

Exemplar genera: Microbotryum Lév. 1847, Ustilentyloma Savile 1964.

Order: Leucosporidiales J. P. Samp., M. Weiß \& R. Bauer, in Sampaio et al., Mycol. Progr. 2: 61 (2003).

Exemplar genera: Leucosporidiella J. P. Samp. 2003, Leucosporidium Fell, Statzell, I. L. Hunter \& Phaff 1970, Mastigobasidium Golubev 1999.

Order: Sporidiobolales J. P. Samp., M. Weiß \& R. Bauer, in Sampaio et al., Mycol. Progr. 2: 66 (2003).

Exemplar genera: Sporidiobolus Nyland 1949, Sporobolomyces Kluyver \& C. B. Niel 1924, Rhodosporidium I. Banno 1967, Rhodotorula F. C. Harrison 1927 pro parte. 


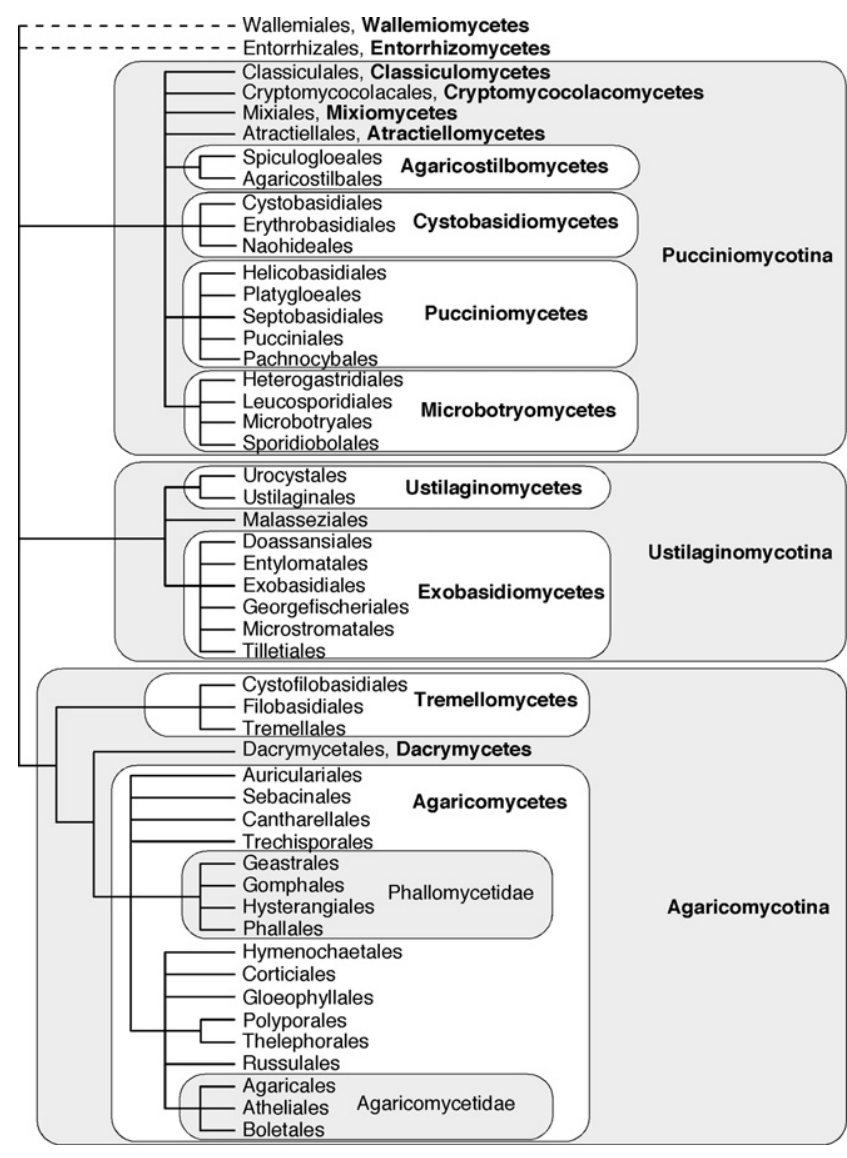

Fig 3 - Phylogeny and classification of Fungi. Basidiomycota. See Table 3 for support values for clades. Dashed lines indicate taxa that are of uncertain placement.

Class: Atractiellomycetes R. Bauer, Begerow, J. P. Samp., M. Weiß \& Oberw., Mycol. Progr. 5: 45 (2006).

Order: Atractiellales Oberw. \& Bandoni, Can. J. Bot. 60: 1740 (1982).

Emend. Oberw. \& Bauer, Sydowia 41: 239 (1989).

Exemplar genera: Atractiella Sacc. 1886, Saccoblastia A. Møller 1895, Helicogloea Pat. 1892, Phleogena Link 1833.

Class: Classiculomycetes R. Bauer, Begerow, J. P. Samp., M. Weiß \& Oberw., Mycol. Progr. 5: 46 (2006).

Order: Classiculales R. Bauer, Begerow, Oberw. \& Marvanová, Mycologia 95: 763 (2003).

Exemplar genera: Classicula R. Bauer, Begerow, Oberw. \& Marvanová 2003, Jaculispora H. J. Huds. \& Ingold 1960.

Class: Mixiomycetes R. Bauer, Begerow, J. P. Samp., M. Weiß \& Oberw., Mycol. Progr. 5: 47 (2006).

Order: Mixiales R. Bauer, Begerow, J. P. Samp., M. Weiß \& Oberw., Mycol. Progr. 5: 47 (2006).

Exemplar genus: Mixia C. L. Kramer 1959 ['1958'].

Class: Cryptomycocolacomycetes R. Bauer, Begerow, J. P. Samp., M. Weiß \& Oberw., Mycol. Progr. 5: 46 (2006).
Order: Cryptomycocolacales Oberw. \& R. Bauer, Mycologia 82: 672 (1990).

Exemplar genera: Cryptomycocolax Oberw. \& R. Bauer 1990, Colacosiphon R. Kirschner, R. Bauer \& Oberw. 2001.

Subphylum: Ustilaginomycotina R. Bauer, Begerow, J. P. Samp., M. Weiß \& Oberw., Mycol. Progr. 5: 45 (2006).

Equivalent to Ustilaginomycetes (Bauer et al. 1997, 2001; Swann \& Taylor 1995).

The classification of Ustilaginomycotina employed here largely parallels that of Begerow et al. (2007), with the primary differences being that here the Entorrhizomycetes are classified as incertae sedis among Basidiomycota (rather than being a class within Ustilaginomycotina).

Class: Ustilaginomycetes R. Bauer, Oberw. \& Vánky, Can. J. Bot. 75: 1311 (1997).

Emend. Begerow, Stoll \& Bauer, Mycologia 98: 908 (2007) ['2006'].

Equivalent to Ustilaginomycetidae Jülich as emmended by Bauer \& Oberwinkler (Bauer et al. 1997, 2001; Weiß et al. 2004a).

Order: Urocystales R. Bauer \& Oberw., in Bauer et al., Can. J. Bot. 75: 1311 (1997).

Exemplar genera: Urocystis Rabenh. ex Fuckel 1870, Ustacystis Zundel 1945, Doassansiopsis (Setch.) Dietel 1897.

Melanotaenium de Bary 1874 has also been placed in this order (Bauer et al. 2001; Weiß et al. 2004a), but analyses of Begerow et al. (2007) and Matheny et al. (2007b) have supported its transfer to Ustilaginales.

Order: Ustilaginales G. Winter, Rabenh. Krypt.-Fl. 2nd ed. 1(1.1): 73 (1880), as 'Ustilagineae'.

Emend. Bauer \& Oberwinkler, in Bauer et al., Can. J. Bot. 75: 1311 (1997).

Exemplar genera: Ustilago (Pers.) Roussel 1806, Cintractia Cornu 1883.

Thecaphora Fingerh. 1836 has also been placed in this order (Bauer et al. 2001), but analyses of Begerow et al. (2007) and Matheny et al. (2007b) have suggested that it is not nested in Ustilaginales. Thecaphora may be the sister group of Urocystales (Matheny et al. 2007b).

Class: Exobasidiomycetes Begerow, Stoll \& R. Bauer, Mycologia 98: 908 (2007) ['2006'].

Equivalent to Exobasidiomycetidae Jülich 1981 emend. Bauer \& Oberwinkler, except for exclusion of Malasseziales (Bauer et al. 1997, 2001; Weiß et al. 2004a).

Monophyly of the Exobasidiomycetidae, as delimited here, is supported with high Bayesian posterior probability in analyses of rpb1, rpb2, and tef1, and nuclear lsu, ssu, and 5.8S ribosomal genes (Matheny et al. 2007b), but it is weakly supported in analyses using atp6, $\beta$-tubulin, and nuc-lsu ribosomal RNA genes (Begerow et al. 2007). See comments regarding Malasseziales.

Order: Doassansiales R. Bauer \& Oberw., in Bauer et al., Can. J. Bot. 75: 1312 (1997). 
Exemplar genera: Doassansia Cornu 1883, Rhamphospora D. D. Cunn. 1888, Nannfeldtiomyces Vánky 1981.

Order: Entylomatales R. Bauer \& Oberw., in Bauer et al., Can. J. Bot. 75: 1311 (1997).

Exemplar genera: Entyloma de Bary 1874, Tilletiopsis Derx 1948.

Begerow et al. (2007) erected the monotypic order Ceraceosorales Begerow, Stoll \& R. Bauer for Ceraceosorus bombacis (B. K. Bakshi) B. K. Bakshi 1976, which was weakly supported as the sister group of Tilletiopsis albescens Gokhale 1972. The Ceraceosorus-T. albescens clade was placed as the sister group of Entylomatales, again with weak support. Ceraceosorales is not included in the present classification, pending more robust resolution of the relationships among Ceraceosorus, Tilletiopsis, and Entyloma.

Order: Exobasidiales Henn., in Engler \& Prantl (eds), Nat. Pflanzenfam. 1(1**): 103 (1897), as 'Exobasidiineae'.

Emend. Bauer, Oberwinkler \& Vánky, Can. J. Bot. 75: 1312 (1997).

Exemplar genera: Exobasidium Woronin 1867, Clinoconidium Pat. 1898, Dicellomyces L. S. Olive 1945.

Order: Georgefischeriales R. Bauer, Begerow \& Oberw., in Bauer et al., Can. J. Bot. 75: 1311 (1997).

Exemplar genera: Georgefischeria Thirum. \& Naras. emend. Gandhe 1980, Phragmotaenium R. Bauer, Begerow, A. Nagler \& Oberw. 2001, Tilletiaria Bandoni \& B. N. Johri 1972, Tilletiopsis Derx 1948 pro parte.

Order: Microstromatales R. Bauer \& Oberw., in Bauer et al., Can. J. Bot. 75: 1311 (1997).

Exemplar genera: Microstroma Niessl 1861, Sympodiomycopsis Sugiy., Tokuoka \& Komag. 1991, Volvocisporium Begerow, R. Bauer \& Oberw. 2001.

Order: Tilletiales Kreisel ex R. Bauer \& Oberw., in Bauer et al., Can. J. Bot. 75: 1311 (1997).

Exemplar genera: Tilletia Tul. \& C. Tul. 1847, Conidiosporomyces Vánky 1992, Erratomyces M. Piepenbr. \& R. Bauer 1997.

Ustilaginomycotina incertae sedis (not placed in any class):

Order: Malasseziales R. T. Moore, Bot. Mar. 23: 371 (1980).

Emend. Begerow, Bauer \& Boekhout, Mycol. Res. 104: 59 (2000).

Exemplar genus: Malassezia Baill. 1889.

Analyses of the protein-coding genes $r p b 1, r p b 2$, and tef1, alone or in combination with nuclear LSU, SSU, and 5.8S ribosomal genes, suggest that Malasseziales are included in the Ustilaginomycetes, but analyses of nuclear ribosomal genes alone or in combination with atp 6 and $\beta$-tubulin suggest that Malasseziales is in the Exobasidiomycetes (Bauer et al. 2001; Begerow et al. 2007; Matheny et al. 2007b; Weiß et al. 2004a).

Subphylum: Agaricomycotina Dowell Prosyllabus LXXVII (2001).

Homonym: Agaricomycotina R. Bauer, Begerow, J. P. Samp., M. Weiß \& Oberw., Mycol. Progr. 5: 45 (2006). Equivalent to
Hymenomycetes (Swann \& Taylor 1995) or Basidiomycetes (Kirk et al. 2001; Hibbett 2007).

Class: Tremellomycetes Dowell, Prosyllabus: LXXVII (2001).

Dimorphic fungi. Fruiting bodies gelatinous or absent, parenthesomes sacculate or absent, basidia septate or nonseptate. The least inclusive clade containing Tremellales, Filobasidiales and Cystofilobasidiales.

Equivalent to Tremellomycetidae sensu Swann \& Taylor (1995) and Weiß et al. (2004a). The name Tremellomycetidae was earlier published by Locquin (1984), but without a Latin diagnosis, and it is therefore invalid under the Code.

Order: Cystofilobasidiales Fell, Roeijmans \& Boekhout, Int. J. Syst. Bacteriol. 49: 911 (1999).

Exemplar genera: Cystofilobasidium Oberw. \& Bandoni 1983, Mrakia Y. Yamada \& Komag. 1987, Itersonilia Derx 1948.

Order: Filobasidiales Jülich, Biblthca Mycol. 85: 324 (1981).

Exemplar genera: Filobasidiella Kwon-Chung 1976, Cryptococcus Vuill. 1901 ( pro parte).

Order: Tremellales Fr., Syst. Mycol. 1: 2 (1821), as 'Tremellinae'.

As delimited here, the group includes Trichosporonales Boekhout \& Fell 2001 (Fell et al. 2001) and Christianseniales F. Rath 1991 (Wells \& Bandoni 2001). Filobasidiales, which Weiß et al. (2004a) included in Tremellales s. lat., has been resolved as the sister group of Tremellales (Fell et al. 2001; Matheny et al. 2007b; Swann \& Taylor 1995).

Exemplar genera: Tremella Pers. 1794, Trichosporon Behrend 1890, Christiansenia Hauerslev 1969.

Class: Dacrymycetes Dowell, Prosyllabus: LXXVII (2001)

Fruiting bodies gelatinous, basidia furcate (rarely unisporous), parenthesomes imperforate.

Containing the single order Dacrymycetales (Wells \& Bandoni 2001).

Order: Dacrymycetales Henn., in Engler \& Prantl (eds), Nat.

Pflanzenfam. 1(1**): 96 (1898), as 'Dacryomycetineae'.

Exemplar genera: Dacrymyces Nees 1861, Calocera (Fr.) Fr. 1828, Guepiniopsis Pat. 1883.

Class: Agaricomycetes Dowell, Prosyllabus: LXXVII (2001)

Fruiting bodies hymenomycetous or gasteroid, basidia two- to eight-spored, parenthesomes perforate or imperforate. The least-inclusive clade containing Auriculariales, Sebacinales, Cantharellales, Phallomycetidae and Agaricomycetidae.

This group is approximately equivalent to Homobasidiomycetes sensu Hibbett \& Thorn (2001) plus Auriculariales and Sebacinales.

Subclass: Agaricomycetidae Parmasto, Windahlia 16: 16 (1986). Synonym: Basidiosporeae Bessey, Univ. Studies, Univ. Nebr.

7: 306 (1907).

The least-inclusive clade containing Agaricales, Boletales and Atheliales.

The delimitation of Agaricomycetidae adopted here differs from that of Parmasto (1986), who described Agaricomycetidae 
as a subclass of Cantharellomycetes Parm. 1986. For example, many of the resupinate forms in the Agaricomycetidae were placed by Parmasto in the Corticiomycetes Parm. 1986. The name Agaricomycetidae was also published by Locquin (1984), but without a Latin diagnosis and it is therefore invalid under the Code.

Order: Agaricales Underw., Moulds, Mildews Mushrooms: 97 (1899).

Equivalent to euagarics clade (Hibbett \& Thorn 2001).

Exemplar genera: Agaricus L. 1753, Coprinus Pers. 1797, Pleurotus (Fr.) P. Kumm. 1871.

Order: Atheliales Jülich, Biblthca Mycol. 85: 343 (1981).

Equivalent to athelioid clade (Binder et al. 2005; Larsson et al. 2004).

Exemplar genera: Athelia Pers. 1822, Piloderma Jülich 1969, Tylospora Donk 1960.

Order: Boletales E.-J. Gilbert, Liures Mycol. 3: 83 (1931).

Equivalent to bolete clade (Binder \& Hibbett 2006; Hibbett \& Thorn 2001).

Exemplar genera: Boletus Fr. 1821, Scleroderma Pers. 1801, Coniophora DC. 1815, Rhizopogon Fr. \& Nordholm 1817.

Subclass: Phallomycetidae K. Hosaka, Castellano \& Spatafora, Mycologia 98: 955 (2007) ['2006'].

Equivalent to Phallales sensu Kirk et al. (2001), and the gomphoid-phalloid clade (Hibbett \& Thorn 2001; Hosaka et al. 2007).

Order: Geastrales K. Hosaka \& Castellano, Mycologia 98: 957 (2007) ['2006'].

Exemplar genera: Geastrum Pers. 1794, Radiigera Zeller 1944, Sphaerobolus Tode 1790.

Order: Gomphales Jülich, Biblthca Mycol. 85: 348 (1981).

Exemplar genera: Gomphus (Fr.) Weinm. 1826, Gautieria Vittad. 1831, Ramaria Holmsk. 1790.

Order: Hysterangiales K. Hosaka \& Castellano, Mycologia 98: 956 (2007) ['2006'].

Exemplar genera: Hysterangium Vittad. 1831, Phallogaster Morgan 1893, Gallacea Lloyd 1905, Austrogautieria E. L. Stewart \& Trappe 1985.

Order: Phallales E. Fisch., in Engler \& Prantl (eds), Nat. Pflanzenfam. 1(1**): 276 (1898).

Equivalent to Phallomycetidae Locq. (Locquin 1984), which was invalidly published, owing to the absence of a Latin diagnosis.

Exemplar genera: Phallus Junius ex L. 1753, Clathrus P. Micheli ex L. 1753, Claustula K. M. Curtis 1926.

Agaricomycetes incertae sedis (not placed in any subclass):

Order: Auriculariales J. Schröt., in Cohn (ed.), Krypt.-Fl. Schlesien 1: 382 (1889).

Exemplar genera: Auricularia Bull. ex Juss. 1789, Exidia Fr. 1822, Bourdotia (Bres.) Trotter 1913.
Order: Cantharellales Gäum., Vergl. Morph. Pilze: 495 (1926).

Equivalent to the cantharelloid clade (Hibbett \& Thorn 2001; Moncalvo et al. 2007). The Cantharellales as delimited here includes Tulasnella, which is distinguished by unusual basidia with inflated sterigmata, and has been classified in a separate order, Tulasnellales Rea 1922 (e.g. Weiß et al. 2004a). Extreme evolutionary rate heterogeneity in the nuclear ribosomal RNA genes of Tulasnella, Cantharellus and Craterellus is a source of error in phylogenetics of Cantharellales. Analyses of Matheny et al. (2006) suggest that Tulasnella is nested within the Cantharellales, but it could also be the sister group to Cantharellales s.str. (Moncalvo et al. 2007). If so, then it may be appropriate to segregate Tulasnellales from Cantharellales s.str.

Exemplar genera: Cantharellus Fr. 1821, Botryobasidium Donk 1931, Craterellus Pers. 1825, Tulasnella J. Schröt. 1888.

Order: Corticiales K. H. Larss., ord. nov. MycoBank no.: MB 501299

Basidiomata resupinata, effuso-reflexa vel discoidea; hymenophora laevia; systema hypharum monomiticum; dendrohyphidia raro absentia; basidia saepe e probasidiis oriuntur. Cystidia presentia vel absentia. Sporae hyalinae, tenuitunicatae, albae vel aggregatae roseae.

Typus: Corticium Pers. 1794.

Basidiomycetes with effused or discoid (Cytidia) basidiomata, a smooth hymenophore, and a monomitic hyphal system with clamped, rarely simple-septate, hyphae. Dendrohyphidia common. Species with or without cystidia. A probasidial resting stage is present in many species. Spores smooth, in masses white to pink. Saprotrophic, parasitic, or lichenicolous.

Equivalent to Vuilleminiales Boidin et al. 1998 and the corticioid clade (Binder et al. 2005; Larsson et al. 2004). Boidin et al. (1998) explicitly included Corticium in their new order, as a member of the family Vuilleminiaceae Maire 1902. Jülich (1981) also placed Corticium in Vuilleminiaceae but referred them to Aleurodiscales Jülich 1981. Corticium is the type of Corticiaceae Herter 1910, a family name conserved against Vuilleminiaceae. The introduction of Corticiales as a new name for this order is, therefore, the preferred option.

Exemplar genera: Corticium Pers. 1794, Vuilleminia Maire 1902, Punctularia Pat. 1895.

Order: Gloeophyllales Thorn, ord. nov. MycoBank no.: MB 501300

Basidiomata annua vel perennia, resupinata, effuso-reflexa, dimidiata vel pileata; hymenophora laevia, merulioidea, odontioidea vel poroidea. Systema hypharum monomiticum, dimiticum vel trimiticum. Hyphae generativae fibulatae vel efibulatae. Leptocystidia ex trama in hymenium projecta, hyalina vel brunnea, tenuitunicata vel crassitunicata. Basidiosporae laeves, hyalinae, tenuitunicatae, ellipsoideae vel cylindricae vel allantoideae, inamyloideae. Lignum decompositum brunneum vel album.

Typus: Gloeophyllum P. Karst. 1882.

Fruiting bodies perennial or annual and long-lived, with hymenium maturing and thickening over time. Stature resupinate, effused-reflexed or dimidiate, with smooth, wrinkled, 
dentate, lamellate or regularly poroid hymenophore, or pileate-stipitate with lamellae. (Aborted, coralloid or flabelliform fruiting bodies may be formed under conditions of darkness or high carbon dioxide concentration). Leptocystidia or hyphoid hairs originating in the context and extending into or protruding from the hymenial layer (or lamellar margin in Neolentinus) are common; these often with thick brown walls and brownish incrustation. Context brown (but pallid in Neolentinus) and generally darkening in potassium hydroxide (the brownish incrustation in Boreostereum turning green in potassium hydroxide). Monomitic (if so, with sclerified generative hyphae), dimitic, or trimitic; generative hyphae with or without clamp connections. Basidiospores hyaline, ellipsoid to cylindrical or suballantoid, with thin, smooth walls, and neither amyloid, dextrinoid nor cyanophilous. Where this is known, basidiospores are binucleate and sexuality is heterothallic and bipolar (but tetrapolar in V. berkeleyi).

Causing brown rots (Gloeophyllum, Neolentinus, Veluticeps) or stringy white rot (Boreostereum, Donkioporia) of wood of gymnosperms, monocots and dicots. Occurrence on 'wood in service' (e.g. railway ties, paving blocks, wooden chests) seems to be common (in Donkioporia, Gloeophyllum, Heliocybe and Neolentinus); often on charred wood (Boreostereum and Veluticeps).

Equivalent to Gloeophyllum clade (Binder et al. 2005).

Exemplar genera: Gloeophyllum P. Karst. 1882, Neolentinus Redhead \& Ginns 1985, Veluticeps (Cooke) Pat. 1894.

Order: Hymenochaetales Oberw., in Frey et al. (eds), Beitr. Biol. niederen Pflanz.: 89 (1977).

Equivalent to the hymenochaetoid clade (Hibbett \& Thorn 2001; Larsson et al. 2007).

Exemplar genera: Hymenochaete Lév. 1846, Phellinus Quél. 1886, Trichaptum Murrill 1904.

Order: Polyporales Gäum., Vergl. Morph. Pilze: 503 (1926). Equivalent to polyporoid clade (Hibbett \& Thorn 2001). Exemplar genera: Polyporus Fr. 1815, Fomitopsis P. Karst. 1881, Phanerochaete P. Karst. 1889.

Order: Russulales Kreisel ex P. M. Kirk, P. F. Cannon \& J. C. David, in Kirk et al., Ainsworth \& Bisby's Dict. Fungi (9th edn): xi (2001).

Equivalent to the russuloid clade (Hibbett \& Thorn 2001;

Larsson \& Larsson 2003; Miller et al. 2007).

Exemplar genera: Russula Pers. 1796, Aleurodiscus Rabenh. ex J. Schröt. 1888, Bondarzewia Singer 1940, Hericium Pers. 1794, Peniophora Cooke 1879, Stereum Pers. 1794.

Order: Sebacinales M. Weiß, Selosse, Rexer, A. Urb. \& Oberw., Mycol. Res. 108: 1007 (2004b).

Exemplar genera: Sebacina Tul. 1871, Tremellodendron G. F. Atk. 1902, Piriformospora Sav. Verma, Aj. Varma, Rexer, G. Kost \& P. Franken 1998.

Order: Thelephorales Corner ex Oberw., Sydowia 78: 361 (1976). Equivalent to the thelephoroid clade (Hibbett \& Thorn 2001). Exemplar genera: Thelephora Ehrh. ex Willd. 1787, Bankera Coker \& Beers ex Pouzar 1955, Polyozellus Murrill 1910.
Order: Trechisporales K. H. Larss., ord. nov. MycoBank no.: MB 501301

Basidiomata resupinata, stipitata vel clavarioidea. Hymenophora laevia, grandinioidea, hydnoidea vel poroidea. Systema hypharum monomiticum vel dimiticum. Hyphae fibulatae, septa hypharum interdum inflata (ampullata). Cystidia praesentia vel absentia. Basidia 4-6 sterigmata formantia. Sporae laeves vel ornatae. Species lignicolae vel terricolae.

Typus: Trechispora P. Karst. 1890.

Basidiomycetes with effused, stipitate or clavarioid basidiomata. Hymenophore smooth, grandinioid, hydnoid or poroid. Hyphal system monomitic, hyphae clamped, subicular hyphae with or without ampullate septa. Cystidia present in some species, mostly lacking. Basidia with four to six sterigmata. Spores smooth or ornamented. On wood or soil.

Equivalent to Hydnodontales Jülich 1981 and trechisporoid clade (Binder et al. 2005; Larsson et al. 2004). Hydnodon Banker 1913 was recently placed in synonomy under Trechispora (Ryvarden 2002) and this synonomy is supported by molecular data (K.H. Larsson, unpubl.). The introduction of a new name for the group, a name that connects to the clade name already established and that is based on the most species-rich genus is, therefore, justified.

Exemplar genera: Trechispora P. Karst. 1890, Sistotremastrum J. Erikss. 1958, Porpomyces Jülich 1982.

Basidiomycota incertae sedis (not placed in any subphylum):

Class: Wallemiomycetes Zalar, de Hoog \& Schroers, Antonie van Leeuwenhoek 87: 322 (2005).

Analyses of rpb1, rpb2, tef1, and nuc-lsu, nuc-ssu, and 5.8S ribosomal RNA genes suggest that the Wallemiomycetes is the sister group of the rest of the Basidiomycota (possibly along with Entorrhizomycetes, see below), but subsets of this dataset produce alternative placements (Matheny et al. 2007b; Zalar et al. 2005).

Order: Wallemiales Zalar, de Hoog \& Schroers, Antonie van Leeuwenhoek 87: 322 (2005).

Exemplar genus: Wallemia Johan-Olsen 1887.

Class: Entorrhizomycetes Begerow, Stoll \& R. Bauer, Mycologia 98: 908 (2007) ['2006'].

Equivalent to Entorrhizomycetidae R. Bauer \& Oberw. (Bauer et al. 1997). So far, only ribosomal RNA genes have been sequenced in Entorrhizomycetes. Analyses with broad sampling across all groups of Basidiomycota and including Ascomycota and Glomeromycota as outgroups suggest that Entorrhizomycetes is not nested within any subphylum, and may be the sister group of the rest of the Basidiomycota (Matheny et al. 2007a; also see Begerow et al. 1997).

Order: Entorrhizales R. Bauer \& Oberw., in Bauer et al., , Can. J. Bot. 75: 1311 (1997).

Exemplar genus: Entorrhiza C. A. Weber 1884. 


\section{Acknowledgments}

Preparation of this classification was facilitated by the Assembling the Fungal Tree of Life (AFTOL) project and the Deep Hypha Research Coordination Network, which were supported by the US National Science Foundation grants DEB-0228657, DEB-0228668, DEB-0228671, DEB-0228725 and NSF-0090301.

\section{Note added in proof}

After this article went to press, the authors became aware of the following publication, which includes alternative citations for many of the names included here: Doweld A, 2001. Prosyllabus tracheophytorum: Tentamen systematis plantarum vascularium (Tracheophyta). Geos, Moscow.

\section{REFERENCE S*}

Adl SM, Simpson AGB, Farmer MA, Andersen RA, Anderson OR, Barta JR, Bowser SS, Brugerolle G, Fensome RA, Fredericq S, James TY, Karpov S, Kugrens P, Krug J, Lane CE, Lewis LA, Lodge J, Lynn DH, Mann DG, McCourt RM, Mendoza L, Moestrup $\varnothing$, Mozley-Standridge SE, Nerad TA, Shearer CA, Smirnov AV, Spiegel FW, Taylor MFJR, 2005. The new higher level classification of eukaryotes with emphasis on the taxonomy of protists. Journal of Eukaryotic Microbiology 52: 399451.

Aime MC, Matheny PB, Henk DA, Frieders EM, Nilsson RH, Piepenbring M, McLaughlin DJ, Szabo LJ, Begerow D, Sampaio JP, Bauer R, Weiß M, Oberwinkler F, Hibbett DS, 2007 ['2006']. . An overview of the higher-level classification of Pucciniomycotina based on combined analyses of nuclear large and small subunit rDNA sequences. Mycologia 98: 896-905.

Alexopoulos CJ, Mims CW, Blackwell M, 1996. Introductory Mycology, fourth ed. John Wiley, New York.

von Arx JA, 1967. Pilzkunde. J. Cramer, Lehre.

Balbiani G, 1882. Sur les microsporides ou psorospermies des Articulés. Comptes rendus de l'Académie des Sciences, Paris 95: 1168-1171.

Baldauf SL, Roger AJ, Wenk-Siefert I, Doolittle WF, 2000. A kingdom-level phylogeny of eukaryotes based on combined protein data. Science 290: 972-977.

Barr DJS, 1980. An outline for the reclassification of the Chytridiales, and for a new order, the Spizellomycetales. Canadian Journal of Botany 58: 2380-2394.

Barr ME, 1983. The ascomycete connection. Mycologia 75: 1-13.

Barr ME, 1987a. New taxa and combinations in the Loculoascomycetes. Mycotaxon 29: 501-505.

Barr ME, 1987b. Prodromus to Class Loculoascomycetes. University of Massachusetts, Amherst.

Barron GL, 1975. Nematophagus fungi: Helicocephalum. Transactions of the British Mycological Society 65: 309-310.

Bauer R, Begerow D, Oberwinkler F, Piepenbring M, Berbee ML, 2001. Ustilaginomycetes. In: McLaughlin DJ, McLaughlin EJ, Lemke P (eds), The Mycota. Vol. VIII: Part B. Systematics and Evolution. Springer-Verlag, Berlin, pp. 57-84.

Bauer R, Begerow D, Sampaio JP, Weiß M, Oberwinkler F, 2006. The simple-septate basidiomycetes: a synopsis. Mycological Progress 5: 41-66.

\footnotetext{
* This list of references, in addition to including papers cited in the text, also contains the full bibliographic details of some papers otherwise cited only as places of publications of names where those works may otherwise be difficult to locate.
}

Bauer R, Oberwinkler F, Vánky K, 1997. Ultrastructural markers and systematics in smut fungi and allied taxa. Canadian Journal of Botany 75: 1273-1314.

Begerow D, Bauer R, Boekhout T, 2000. Phylogenetic placements of ustilaginomycetous anamorphs as deduced from nuclear LSU rDNA sequences. Mycological Research 104: 53-60.

Begerow D, Bauer R, Oberwinkler F, 1997. Phylogenetic studies on nuclear large subunit ribosomal DNA sequences of smut fungi and related taxa. Canadian Journal of Botany 75: 2045-2056.

Begerow D, Stoll M, Bauer R, 2007 ['2006']. A phylogenetic hypothesis of Ustilaginomycotina based on multiple gene analyses and morphological data. Mycologia 98: 906-916.

Benjamin RK, 1979. Zygomycetes and their spores. In: Kendrick B (ed), The Whole Fungus: the Sexual-Asexual Synthesis. National Museum of Natural Sciences, Ottawa, pp. 573-616.

Benny GL, Kimbrough JW, 1980. A synopsis of the orders and families of Plectomycetes with keys to genera. Mycotaxon 12: 1-91.

Benny GL, White MM, 2001. The classification and phylogeny of Trichomycetes and Zygomycetes. In: Misra JK, Horn BW (eds), Trichomycetes and Other Fungal Groups. Science Publishers, Enfield, NH, pp. 39-53.

Berres ME, Szabo LJ, McLaughlin DJ, 1995. Phylogenetic relationships in auriculariaceous basidiomycetes based on $25 \mathrm{~S}$ ribosomal DNA sequences. Mycologia 87: 821-840.

Binder M, Hibbett DS, Larsson K-H, Larsson E, Langer E, Langer G, 2005. The phylogenetic distribution of resupinate forms across the major clades of mushroom-forming fungi (Homobasidiomycetes). Systematics and Biodiversity 3: 1-45.

Binder M, Hibbett DS, 2007 ['2006']. Molecular systematics and biological diversification of Boletales. Mycologia 98: 971-983.

Blackwell M, Hibbett DS, Taylor JW, Spatafora JW, 2007 ['2006']. Research coordination networks: a phylogeny for kingdom Fungi. Mycologia 98: 829-837.

Boidin J, Mugnier J, Canales R, 1998. Taxonomie moléculaire des Aphyllophorales. Mycotaxon 66: 445-492.

Bold HC, 1957. The Morphology of Plants. Harper Row, New York.

Bullerwell CE, Forget L, Lang BF, 2003. Evolution of monoblepharidalean fungi based on complete mitochondrial genome sequences. Nucleic Acids Research 31: 1614-1623.

Campbell J, Anderson JL, Shearer CA, 2003. Systematics of Halosarpheia based on morphological and molecular data. Mycologia 95: 530-552.

Campbell J, Volkmann-Kohlmeyer B, Gräfenhan T, Spatafora JW, Kohlmeyer J, 2005. A re-evaluation of Lulworthiales: relationships based on 18S and 28S rDNA. Mycological Research 109: 556-568.

Castlebury LA, Rossman AY, Jaklitsch WJ, Vasilyeva LN, 2002. A preliminary overview of the Diaporthales based on large subunit nuclear ribosomal DNA sequences. Mycologia 94: 1017-1031.

Castlebury LA, Rossman AY, Sung G-H, Hyten AS, Spatafora JW, 2004. Multigene phylogeny reveals new lineage for Stachybotrys chartarum, the indoor air fungus. Mycological Research 108 : 864-872.

Cavalier-Smith T, 1981. Eukaryote kingdoms: seven or nine? BioSystems 14: 461-481.

Cavalier-Smith T, 1998. A revised six-kingdom system of Life. Biological Reviews 73: 203-266.

Cejp K, 1957. Houby; celostátní vysokoškolská učebnice. Vol. 1. Czechoslovakian Academy of Sciences Press, Prague.

Clements FE, Shear CL, 1931. The Genera of Fungi, second ed. H.W. Wilson, New York.

Cohn F, 1879. Ueber ein Thallophytensystem. Jahresbericht der Schlesischen Gesellschaft für vaterländische Cultur, Breslau 57: 279.

Cracraft J, Donoghue MJ (eds), 2004. Assembling the Tree of Life. Oxford University Press, Oxford.

Currah RS, 1985. Taxonomy of the Onygenales: Arthrodermataceae, Gymnoacaceae, Myxotrichaceae and Onygenaceae. Mycotaxon 24: $1-216$. 
David JC, 2002. A preliminary catalogue of the names of fungi above the rank of order. Constancea 83: 1-30.

Del Prado R, Schmitt I, Kautz S, Palice Z, Luecking R, Lumbsch HT, 2006. Molecular data place Trypetheliaceae in Dothideomycetes. Mycological Research 110: 511-520.

Donk MA, 1964. A conspectus of the families of Aphyllophorales. Persoonia 3: 199-324.

Ekman S, Tønsberg T, 2002. Most species of Lepraria and Leproloma form a monophyletic group closely related to Stereocaulon. Mycological Research 106: 1262-1276.

Engler A, 1898. Syllabus der Pflanzenfamilien: Eine Übersicht über das gesammte Pflanzensystem, second ed. Gebrüder Borntraeger Verlag, Berlin, 46.

Eriksson O, 1982. Outline of the ascomycetes. Mycotaxon 15: $203-$ 248.

Eriksson OE, 1986. Lahmia Körber (=Parkerella A. Funk) a misinterpreted genus with isolated position. Mycotaxon 27: 347-360.

Eriksson OE, 1992. Huangshania verrucosa gen. et sp. nov. (Triblidiaceae, Triblidiales ordo nov.), a discomycete on Pinus from China. Systema Ascomycetum 11: 1-10.

Eriksson OE, 1994. Pneumocystis carinii, a parasite in lungs of mammals, referred to a new family and order (Pneumocystidaceae, Pneumocystidales, Ascomycota). Systema Ascomycetum 13: 165-180.

Eriksson OE, Baral H-O, Currah RS, Hansen K, Kurtzman CP, Rambold G, Læss øe T, 2003. Notes on ascomycete systematics. Nos 3580-3623. Myconet 9: 91-103.

Eriksson OE, Svedskog A, Landvik S, 1993. Molecular evidence for the evolutionary hiatus between Saccharomyces cerevisiae and Schizosaccharomyces pombe. Systema Ascomycetum 11: 119-162.

Eriksson OE, Winka K, 1997. Supraordinal taxa of Ascomycota. Myconet 1: 1-16.

Fell JW, Boekhout T, Fonseca A, Sampaio JP, 2001. Basidiomycetous yeasts. In: McLaughlin DJ, McLaughlin EJ, Lemke P (eds), The Mycota. Vol. VII. Part B., Systematics and Evolution. SpringerVerlag, Berlin, pp. 3-35.

Fell JW, Roeijmans H, Boekhout T, 1999. a new order of basidiomycetous yeasts. International Journal of Systematic Bacteriology. Cystofilobasidiales 49: 907-913.

Fell JW, Scorzetti G, 2004. Reassignment of the basidiomycetous yeasts Trichosporon pullulans to Guehomyces pullulans gen. nov., comb. nov. and Hyalodendron lignicola to Trichosporon lignicola comb. nov. International Journal of Systematic and Evolutionary Microbiology 54: 995-998.

Fischer A, 1892. Phycomycetes. Rabenhorst's Kryptogamen-Flora von Deutschland, Österreich und der Schweiz 1: 1-128.

Fischer E, 1898. Phallineae. In: Engler A, Prantl K (eds), Die natürlichen Pflanzenfamilien nebst ihren Gattungen und wichtigeren Arten insbesondere der Nutzpflanzen, Vol. 1. Wilhelm Engelmann, Leipzig, pp. 276-295.

Fries EM, 1821. Systema Mycologicum, Vol. 1. Berling, Lund.

Fries EM, 1832. Systema Mycologicum, Vol. 3. Ernest Mauriti, Greifswald.

Galagan JE, Henn MR, Ma L-J, Cuomo CA, Birren B, 2005. Genomics of the fungal kingdom: insights into eukaryotic biology. Genome Research 15: 1620-1631.

Gamundí IJ, 1971. Las Cyttariales sudamericanas. Darwiniana 16: 461-510.

Gäumann E, 1926. Vergleichende Morphologie der Pilze. Gustav Fischer, Jena.

Gäumann E, Dodge CW, 1928. Comparative Morphology of the Fungi. McGraw-Hill, London.

Geiser DM, Gueidan C, Miądlikowska J, Lutzoni F, Kauff F, Hofstetter V, Fraker E, Schoch CL, Tibell L, Untereiner WA, Aptroot A, 2007 ['2006']. Eurotiomycetes: Eurotiomycetidae and Chaetothyriomycetidae. Mycologia 98: 1054-1065.
Germot A, Philipe H, Le Guyader H, 1997. Evidence for loss of mitochondria in microsporidia from a mitochondrial HSP70 in Nosema locustae. Molecular and Biochemical Parasitology 87: 159-168.

Gibson JL, Kimbrough JW, Benny GL, 1986. Ultrastructural observations on Endogonaceae (Zygomycetes). II. Glaziellales ord. nov. and Glaziellaceae fam. nov.: new taxa based upon light and electron microscopic observations of Glaziella aurantiaca. Mycologia 78: 941-954.

Gilbert EJ, 1931. Les Bolets. [Les Liures du Mycologue, Vol. 3]. E. Le François, Paris.

Gill EE, Fast NM, 2006. Assessing the microsporidia-fungi relationship: combined phylogenetic analysis of eight genes. Gene 375: 103-109.

Grube M, Baloch E, Lumbsch HT, 2004. The phylogeny of Porinaceae (Ostropomycetidae) suggests a neotenic origin ofperithecia in Lecanoromycetes. Mycological Research 108: 1111-1118.

Gwynne-Vaughan HCI, 1922. Fungi: Ascomycetes, Ustilaginales, Uredinales. Cambridge University Press, Cambridge.

Hausner G, Reid J, 2004. The nuclear small subunit ribosomal genes of Sphaeronaemella helvellae, Sphaeronaemella fimicola, Gabarnaudia betae, and Cornuvesica falcata: phylogenetic implications. Canadian Journal of Botany 82: 752-762.

Hawksworth DL, Kirk PM, Sutton BC, Pegler DN, 1995. Ainsworth \& Bisby's Dictionary of the Fungi, eighth ed. CAB International, Wallingford.

Henk DA, Weir A, Blackwell M, 2003. Laboulbeniopsis, an ectoparasite of termites newly recognized as a member of the Laboulbeniomycetes. Mycologia 95: 561-564.

Hennings P, 1897. Exobasidiineae. In: Engler A, Prantl K (eds), Die natürlichen Pflanzenfamilien, Vol. 1. Wilhelm Engelmann Verlag, Leipzig, pp. 103-105.

Hennings P, 1898. Dacryomycetineae. In: Engler A, Prantl K (eds), Die Natürlichen Pflanzenfamilien, Vol. 1. Wilhelm Engelmann Verlag, Leipzig, pp. 96-102.

Henssen A, Jahms HM, 1973 [1974]. Lichenes: Eine Einfürhung in die Flechtenkunde. George Thieme, Stuttgart.

Hibbett DS, 2007 ['2006']. A phylogenetic overview of the Agaricomycotina. Mycologia 98: 917-925.

Hibbett DS, Donoghue MJ, 1998. Integrating phylogenetic analysis and classification in fungi. Mycologia 90: 347-356.

Hibbett DS, Thorn RG, 2001. Homobasidiomycetes. In: McLaughlin DJ, McLaughlin EJ, Lemke P (eds), The Mycota. Vol. VII. Part B., Systematics and Evolution. Springer-Verlag, Berlin, pp. 121-168.

Hirt RP, Healy B, Vossbrinck CR, Canning EU, Embley TM, 1997. A mitochondrial Hsp70 orthologue in Vairimorpha necatrix: molecular evidence that Microsporidia once contained mitochondria. Current Biology 7: 995-998.

Hofstetter V, Miądlikowska J, Kauff F, Lutzoni F, 2007. Phylogenetic comparison of protein-coding versus ribosomal RNAcoding sequence data: a case study of the Lecanoromycetes (Ascomycota). Molecular Phylogenetics and Evolution, in press.

de Hoog GS, Göttlich E, Platas G, Genilloud O, Leotta G, Brummelen J, 2005. Evolution, taxonomy and ecology of the genus Thelebolus in Antarctica. Studies in Mycology 51: 33-76.

Hosaka K, Bates ST, Beever RT, Castellano MA, Colgan III W, Domínguez LS, Nouhra ER, Geml J, Giachini AJ, Kenney SR, Simpson NB, Spatafora JW, Trappe JM, 2007 ['2006']. Molecular phylogenetics of the gomphoid-phalloid fungi with an establishment of the new subclass Phallomycetidae and two new orders. Mycologia 98: 949-959.

Huhndorf SM, Miller AN, Fernández FA, 2004a. Molecular systematics of the Coronophorales and new species of 
Bertia, Lasiobertia and Nitschkia. Mycological Research 108: 1384-1398.

Huhndorf SM, Miller AN, Fernández FA, 2004b. Molecular systematics of the Sordariales: the order and the family Lasiosphaeriaceae redefined. Mycologia 96: 368-387.

Inderbitzin P, Landvik S, Abdel-Wahab MA, Berbee ML, 2001. Aliquandostipitaceae, a new family for two new tropical ascomycetes with unusually wide hyphae and dimorphic ascomata. American Journal of Botany 88: 52-61.

Inderbitzin P, Lim SR, Volkmann-Kohlmeyer B, Kohlmeyer J, Berbee ML, 2004. The phylogenetic position of Spathulospora based on DNA sequences from dried herbarium material. Mycological Research 108: 737-748.

International Commission on Zoological Nomenclature, 1999. International Code of Zoological Nomenclature. International Trust for Zoological Nomenclature, London.

Jahn TL, Jahn FF, 1949. How to Know the Protozoa. Wm C. Brown, Dubuque.

James TY, Kauff F, Schoch CL, Matheny PB, Hofstetter V, Cox C, Celio G, Gueidan C, Fraker E, Miądlikowska J, Lumbsch HT, Rauhut A, Reeb V, Arnold EA, Amtoft A, Stajich JE, Hosaka K, Sung G-H, Johnson D, O’Rourke B, Crockett M, Binder M, Curtis JM, Slot JC, Wang Z, Wilson AW, Schüßler A, Longcore JE, O’Donnell K, Mozley-Standridge S, Porter D, Letcher PM, Powell MJ, Taylor JW, White MM, Griffith GW, Davies DR, Humber RA, Morton J, Sugiyama J, Rossman AY, Rogers JD, Pfister DH, Hewitt D, Hansen K, Hambleton S, Shoemaker RA, Kohlmeyer J, Volkmann-Kohlmeyer B, Spotts RA, Serdani M, Crous PW, Hughes KW, Matsuura K, Langer E, Langer G, Untereiner WA, Lücking R, Büdel B, Geiser DM, Aptroot A, Diederich P, Schmitt I, Schultz M, Yahr R, Hibbett DS, Lutzoni F, McLaughlin D, Spatafora J, Vilgalys R, 2006. Reconstructing the early evolution of the fungi using a six gene phylogeny. Nature 443: 818-822.

James TY, Letcher PM, Longcore JE, Mozley-Standridge SE, Porter D, Powell MJ, Griffith GW, Vilgalys R, 2007 ['2006']. A molecular phylogeny of the flagellated fungi (Chytridiomycota) and a proposal for a new phylum (Blastocladiomycota). Mycologia 98: 860-871.

James TY, Porter D, Leander CA, Vilgalys R, Longcore JE, 2000. Molecular phylogenetics of the Chytridiomycota supports the utility of ultrastructural data in chytrid systematics. Canadian Journal of Botany 78: 336-350.

Jülich W, 1981. Higher taxa of basidiomycetes. Bibliotheca Mycologica 85: 1-485.

Kauff F, Lutzoni F, 2002. Phylogeny of the Gyalectales and Ostropales (Ascomycota, Fungi): among and within order relationships based on nuclear ribosomal RNA small and large subunits. Molecular Phylogenetics and Evolution 25: 138-156.

Keeling PJ, 2003. Congruent evidence for alpha-tubulin and betatubulin gene phylogenies for a zygomycete origin of Microsporidia. Fungal Genetics and Biology 38: 298-309.

Keeling PJ, Luker MA, Palmer JD, 2000. Evidence from beta-tubulin phylogeny that Microsporidia evolved from within the fungi. Molecular Biology and Evolution 17: 23-31.

Kendrick B (ed), 1979. The Whole Fungus: the Sexual-asexual Synthesis, Vol. 2. National Museum of Natural Sciences, Ottawa.

Kendrick B, 1985. The Fifth Kingdom. Mycologue Publications, Waterloo.

Kirk PM, Cannon PF, David JC, Stalpers JA (eds), 2001. Ainsworth \& Bisby's Dictionary of the Fungi, ninth ed. CABI Publishing, Wallingford.

Kohlmeyer J, Spatafora JW, Volkmann-Kohlmeyer B, 2000. Lulworthiales, a new order of marine Ascomycota. Mycologia 92: 453-458.

Kruys A, Eriksson OE, Wedin M, 2006. Phylogenetic relationships of coprophilous Pleosporales (Dothideomycetes, Ascomycota), and the classification of some bitunicate taxa of unknown position. Mycological Research 110: 527-536.

Kuramae EE, Robert V, Snel B, Weiß M, Boekhout T, 2006. Phylogenomics reveal a robust fungal tree of life. FEMS Yeast Research 6: 1213-1220.

Kudrjazew W, 1960. Die Systematik der Hefen. Akademie Verlag, Berlin.

Kurtzman CP, Sugiyama J, 2001. Ascomycetous yeasts and yeastlike taxa. In: McLaughlin DJ, McLaughlin EJ, Lemke P (eds), The Mycota. Vol. VII. Part A. Systematics and Evolution. Springer-Verlag, Berlin, pp. 179-200.

Landvik S, Eriksson OE, 1994. Relationship of the genus Glaziella (Ascomycota) inferred from $18 \mathrm{~S}$ rDNA sequences. Systema Ascomycetum 13: 13-23.

Landvik S, Eriksson OE, Berbee ML, 2001. Neolecta - a fungal dinosaur? Evidence from $\beta$-tubulin amino acid sequences. Mycologia 93: 1151-1163.

Landvik S, Eriksson OE, Gargas A, Gustafsson P, 1993. Relationships of the genus Neolecta (Neolectales ordo nov., Ascomycotina) inferred from 18S rDNA sequences. Systema Ascomycetum 11: 107-118.

Larsson E, Larsson K-H, 2003. Phylogenetic relationships of russuloid basidiomycetes with emphasis on aphyllophoralean taxa. Mycologia 95: 1037-1065.

Larsson JIR, 2000. The hyperparasitic microsporidium Amphiacantha longa Caullery et Mesnil, 1914 (Microspora: Metchnikovellidae) - description of the cytology, redescription of the species, emended diagnosis of the genus Amphiacantha and establishment of the new family Amphiacanthidae. Folia Parasitologica 47: 241-256.

Larsson K-H, Larsson E, Kõljalg U, 2004. High phylogenetic diversity among corticioid homobasidiomycetes. Mycological Research 108: 983-1002.

Larsson K-H, Parmasto E, Fischer M, Langer E, Nakasone KK, Redhead SA, 2007 ['2006']. Hymenochaetales: a molecular phylogeny for the hymenochaetoid clade. Mycologia 98: 926936.

Letcher PM, Powell MJ, Churchill PF, Chambers JG, 2006. Ultrastructural and molecular phylogenetic delineation of a new order, the Rhizophydiales (Chytridiomycota). Mycological Research 110: 898-915.

Li JL, Heath IB, Packer L, 1993. The phylogenetic relationships of the anaerobic chytridiomycetous gut fungi (Neocallimasticaceae) and the Chytridiomycota. II. Cladistic analysis of structural data and description of Neocallimasticales ord. nov. Canadian Journal of Botany 71: 393-407.

Lichtwardt RW, Manier JF, 1978. Validation of the Harpellales and Asellariales. Mycotaxon 7: 441-442.

Lindau G, 1896. Hysteriineae. In: Engler A, Prantl K (eds), Die natürlichen Pflanzenfamilien, Vol. 1. Wilhelm Engelmann Verlag, Leipzig, pp. 265-278.

Lindau G, 1897a. Dothideales. In: Engler A, Prantl K (eds), Die natürlichen Pflanzenfamilien, Vol. 1. Wilhelm Engelmann Verlag, Leipzig, pp. 373-383.

Lindau G, 1897b. Hypocreales. In: Engler A, Prantl K (eds), Die natürlichen Pflanzenfamilien, Vol. 1(1). Leipzig,. Wilhelm Engelmann Verlag, pp. 343-372.

Lindau G, 1897c. Laboulbeniineae. In: Engler A, Prantl K (eds), Die natürlichen Pflanzenfamilien, Vol. 1(1). Leipzig,. Wilhelm Engelmann Verlag, pp. 491-505.

Lindemuth R, Wirtz N, Lumbsch HT, 2001. Phylogenetic analysis of nuclear and mitochondrial rDNA sequences supports the view that loculoascomycetes (Ascomycota) are not monophyletic. Mycological Research 105: 1176-1181.

Liu YJ, Hall BD, 2004. Body plan evolution of ascomycetes, as inferred from an RNA polymerase II phylogeny. Proceedings of the National Academy of Sciences, USA 101: 4507-4512. 
Liu YJ, Hodson MC, Hall BD, 2006. Loss of the flagellum happened only once in the fungal lineage: phylogenetic structure of kingdom Fungi inferred from RNA polymerase II subunit genes. BMC Evolutionary Biology 6: 74.www.biomedcentral. com/1471-2148/6/74.

Locquin M, 1984. Mycologie Générale et Structurale. Masson, Paris.

Lücking R, Stuart B, Lumbsch HT, 2004. Phylogenetic relationships of Gomphillaceae and Asterothyriaceae: evidence from a combined Bayesian analysis of nuclear and mitochondrial sequences. Mycologia 96: 283-294.

Lumbsch T, Palice Z, Wiklund E, Ekman S, Wedin M, 2004. Supraordinal phylogenetic relationships of Lecanoromycetes based on Bayesian analysis of combined nuclear and mitochondrial sequences. Molecular Phylogenetics and Evolution 31: 822-832.

Lumbsch HT, Schmitt I, Lindemuth R, Miller A, Mangold A, Fernandez F, Huhndorf S, 2005. Performance of four ribosomal DNA regions to infer higher-level phylogenetic relationships of inoperculate euascomycetes (Leotiomyceta). Molecular Phylogenetics and Evolution 34: 512-524.

Lumbsch HT, Schmitt I, Lücking R, Wiklund RE, Wedin M, 2007. The phylogenetic placement of Ostropales within Lecanoromycetes (Ascomycota) revisited. Mycological Research 111.

Lumbsch HT, Wirtz N, Lindemuth R, Schmitt I, 2002. Higher level phylogenetic relationships of euascomycetes (Pezizomycotina) inferred from a combined analysis of nuclear and mitochondrial sequence data. Mycological Progress 1: 57-70.

Lutzoni F, Kauff F, Cox CJ, McLaughlin D, Celio G, Dentinger B, Padamsee M, Hibbett DS, James TY, Baloch E, Grube M, Reeb V, Hofstetter V, Schoch C, Arnold AE, Miądlikowska J, Spatafora J, Johnson D, Hambleton S, Crockett M, Shoemaker R, Sung G-H, Lücking R, Lumbsch T, O’Donnell K, Binder M, Diederich P, Ertz D, Gueidan C, Hansen K, Harris RC, Hosaka K, Lim Y-W, Matheny B, Nishida H, Pfister D, Rogers J, Rossman A, Schmitt I, Sipman H, Stone J, Sugiyama J, Yahr R, Vilgalys R, 2004. Assembling the fungal tree of life: progress, classification, and evolution of subcellular traits. American Journal of Botany 91: 1446-1480.

Manier J-F, 1973. Quelques aspects ultrastructuraux du trichomycète asellariale, Asellaria ligae Tuzet et Manier, 1950 ex Manier, 1958. Comptes Rendu hebdomadaires des séances de l’Académie des Sciences de Paris, séries D 276: 3429-3431.

Margulis L, Corliss JO, Melkonian M, Chapman DJ, 1990. Handbook of Protoctista: the structure, cultivation, habitats and life histories of the eukaryotic micro-organisms and their descendants. Jones \& Bartlett, Boston.

Matheny PB, Curtis JM, Hofstetter V, Aime MC, Moncalvo J-M, Ge Z-W, Yang Z-L, Slot JC, Ammirati JF, Baroni TJ, Bougher NL, Hughes KW, Lodge DJ, Kerrigan RW, Seidl MT, Aanen DK, DeNitis M, Daniele GM, Desjardin DE, Kropp BR, Norvell LL, Parker A, Vellinga EC, Vilgalys R, Hibbett DS, 2007 ["2006"]a. Major clades of Agaricales: a multi-locus phylogenetic overview. Mycologia 98: 984-997.

Matheny PB, Gossman JA, Zalar P, Arun Kumar TK, Hibbett DS, 2007a. Resolving the phylogenetic position of the Wallemiomycetes: an enigmatic major lineage of Basidiomycota. Canadian Journal of Botany 84: 1794-1805.

Matheny PB, Wang Z, Binder M, Curtis JM, Lim YW, Nilsson RH, Hughes KW, Hofstetter V, Ammirati JF, Schoch CL, Langer GE, McLaughlin DJ, Wilson AW, Frøslev T, Ge ZW, Kerrigan RW, Slot JC, Vellinga EC, Liang ZL, Baroni TJ, Fischer M, Hosaka K, Matsuura K, Seidl MT, Vaura J, Hibbett DS, 2006. Contributions of $r p b 2$ and tef1 to the phylogeny of mushrooms and allies (Basidiomycota, Fungi). Molecular Phylogenetics and Evolution in press.

McLaughlin DJ, McLaughlin EG, Lemke PA (eds), 2001a. The Mycota. Vol. VII. Part A. Systematics and Evolution. SpringerVerlag, Berlin.
McLaughlin DJ, McLaughlin EG, Lemke PA (eds), 2001b. The Mycota. Vol. VII. Part B. Systematics and Evolution. Springer-Verlag, Berlin. McNeill JF, Barrie F, Burdet HM, Demoulin V, Hawksworth DL, Marhold K, Nicolson DH, Prado J, Silva PC, Skog JE, Wiersema J, Turland NJ (eds), 2006. International Code of Botanical Nomenclature (Vienna Code) [Regnum Vegetabile Vol. 146.]. A.R.G. Ganter Verlag, Ruggell.

Miądlikowska J, Kauff F, Hofstetter V, Fraker E, Reeb V, Grube M, Hafellner J, Kukwa M, Lücking R, Hestmark G, Otalora MG, Rauhut A, Büdel B, Scheidegger C, Timdal E, Stenroos S, Brodo I, Perlmutter GB, Ertz D, Diederich P, Lendemer JC, May P, Schoch CL, Arnold AE, Hodkinson BP, Gueidan C, Tripp E, Yahr R, Robertson C, Lutzoni F, 2007 ['2006']. New insights into classification and evolution of the Lecanoromycetes (Pezizomycotina, Ascomycota) from phylogenetic analyses of three ribosomal RNA- and two protein-coding genes. Mycologia 98: 1089-1102.

Miądlikowska J, Lutzoni F, 2004. Phylogenetic classification of peltigeralean fungi (Peltigerales, Ascomycota) based on ribosomal RNA small and large subunits. American Journal of Botany 91: 449-464.

Miller AN, Huhndorf SM, 2004. A natural classification of Lasiosphaeria based on nuclear LSU rDNA sequences. Mycological Research 108: 26-34.

Miller AN, Huhndorf SM, 2005. Multi-gene phylogenies indicate ascomal wall morphology is a better predictor of phylogenetic relationships than ascospore morphology in the Sordariales (Ascomycota, Fungi). Molecular Phylogenetics and Evolution 35: 60-75.

Miller SL, Larsson E, Larsson K-H, Verbeken A, Nuytinck J, 2007 ['2006']. Perspectives in the new Russulales. Mycologia 98: 960-970.

Moncalvo J-M, Nilsson RH, Koster B, Dunham SM, Bernauer T, Matheny PB, McLenon T, Margaritescu S, Weiß M, Garnica S, Danell E, Langer G, Langer E, Larsson E, Larsson K-H, Vilgalys R, 2007 ['2006']. The cantharelloid clade: dealing with incongruent gene trees and phylogenetic reconstruction methods. Mycologia 98: 937-948.

Moncalvo J-M, Vilgalys R, Redhead SA, Johnson JE, James TY, Aime MC, Hofstetter V, Verduin SJW, Larsson E, Baroni TJ, Thorn RG, Jacobsson S, Clémençon H, Miller Jr OK, 2002. One hundred seventeen clades of euagarics. Molecular Phylogenetics and Evolution 23: 357-400.

Moore RT, 1980. Taxonomic proposals for the classification of marine yeasts and other yeast-like fungi including the smuts. Botanica Marina 23: 361-373.

Moore RT, 1990. Order Platygloeales ord. nov. Mycotaxon 39: 245-248.

Moreau F, 1954. Les Champignons. Physiologie, morphologie, développment et systématique. Vol. 2. Lechevalier, Paris.

Morton JB, Benny GL, 1990. Revised classification of arbuscular mycorrhizal fungi (Zygomycetes): a new order, Glomales, two new suborders, Glomineae and Gigasporineae, and two new families, Acaulosporaceae and Gigasporaceae, with an emendation of Glomaceae. Mycotaxon 37: 471-491.

Moss ST, 1975. Septal structure in the Trichomycetes with special reference to Astreptonema gammari (Eccrinales). Transactions of the British Mycological Society 65: 115-127.

Nagahama T, Hamamoto M, Nakase T, Shimamura S, Horikoshi K, 2006. Phylogenetic relationship within the Erythrobasidium clade: molecular phylogenies, secondary structure, and intron positions inferred from partial sequences of ribosomal RNA and elongation factor-1 $\alpha$ genes. Journal of General and Applied Microbiology 52: 37-45.

Nagahama T, Sato H, Shimazu M, Sugiyama J, 1995. Phylogenetic divergence of the entomophthoralean fungi: evidence from nuclear 18S ribosomal RNA gene sequences. Mycologia 87: 203-209. 
Nannfeldt JA, 1932. Studien über die Morphologie und Systematik der nicht-lichenisierten inoperculaten Discomyceten. Nova Acta Regiae Societatis Scientarum Upsaliensis, ser. 4, 8 (2): 1-368.

Nishida H, Sugiyama J, 1994. Archiascomycetes: detection of a major new lineage within the Ascomycota. Mycoscience 35: 361-366.

Oberwinkler F, 1976. Eine agaricoide Gattung der Thelephorales. Sydowia 78: 359-361.

Oberwinkler F, 1977. Das neue System der Basidiomyceten. In: Frey W, Hurka H, Oberwinkler F (eds), Beiträge zur Biologie der niederen Pflanzen. Gustav Fischer Verlag, Stuttgart, pp. 59-105.

Oberwinkler F, Bandoni RJ, 1982. A taxonomic survey of the gasteroid, auricularioid Heterobasidiomycetes. Canadian Journal of Botany 60: 1726-1750.

Oberwinkler F, Bauer R, 1989. The systematics of gasteroid, auricularioid Heterobasidiomycetes. Sydowia 41: 224-256.

Oberwinkler F, Bauer R, 1990. Cryptomycocolax: a new mycoparasitic heterobasidiomycete. Mycologia 82: 671-692.

O’Donnell K, Cigelnik E, Benny GL, 1998. Phylogenetic relationships among the Harpellales and Kickxellales. Mycologia 90: 286-297.

Pang KL, Abdel-Wahab MA, Sivichai S, El-Sharouney HM, Jones EBG, 2002. Jahnulales (Dothideomycetes, Ascomycota): a new order of lignicolous freshwater ascomycetes. Mycological Research 106: 1031-1042.

Parmasto E, 1986. On the origin of the hymenomycetes (what are corticioid fungi?). Windahlia 16: 3-20.

Peyretaillade E, Broussolle V, Peyret P, Méténier G, Gouy M, Vivarès $C P, 1998$. Microsporidia, amitochondrial protists, possess a $70-\mathrm{kDa}$ heat shock protein gene of mitochondrial evolutionary origin. Molecular Biology and Evolution 15: 683-689.

Réblová M, 2006. Molecular systematics of Ceratostomella sensu lato and morphologically similar fungi. Mycologia 98: 68-93.

Réblová M, Mostert L, Gams W, Crous PW, 2004. New genera in the Calosphaeriales: Togniniella and its anamorph Phaeocrella, and Calosphaeriophora as anamorph of Calosphaeria. Studies in Mycology 50: 533-550.

Réblová M, Seifert KA, 2004. Cryptadelphia (Trichosphaeriales), a new genus for holomorphs with Brachysporium anamorphs and clarification of the taxonomic status of Wallrothiella. Mycologia 96: 343-367.

Reeb V, Lutzoni F, Roux C, 2004. Multilocus phylogenetic circumscription of the lichen-forming fungi family Acarosporaceae and its position within the Ascomycota. Molecular Phylogenetics and Evolution 32: 1036-1060.

Robbertse B, Reeves J, Schoch C, Spatafora JW, 2006. A phylogenomic analysis of the Ascomycota. Fungal Genetics and Biology 43: 715-725.

Rossman AY, Aime MC, Farr DF, Castelbury LA, Peterson KR, Leahy R, 2004. The coelomycetous genera Chaetomella and Pilidium represent a newly discovered lineage of inoperculate discomycetes. Mycological Progress 3: 275-290.

Ryvarden L, 2002. A note on the genus Hydnodon Banker. Synopsis Fungorum 15: 31-33.

Saenz GS, Taylor JW, 1999. Phylogenetic relationships of Meliola and Meliolina inferred from nuclear small subunit rRNA sequences. Mycological Research 103: 1049-1056.

Saikawa M, Sugiura K, Sato H, 1997. Electron microscopy of two trichomycetous fungi attached to the hindgut lining of pillbugs. Canadian Journal of Botany 75: 1479-1484.

Sampaio JP, Gadanho M, Bauer R, Weiß M, 2003. Taxonomic studies in the Microbotryomycetidae: Leucosporidium golubevii sp. nov., Leucosporidiella gen. nov. and the new orders Leucosporidiales and Sporidiobolales. Mycological Progress 2: 53-68.

Sampaio JP, 2004. Diversity, phylogeny and classification of basidiomycetous yeasts. In: Agerer R, Piepenbring M, Blanz P (eds), Frontiers in Basidiomycote Mycology. IHW Verlag, Eching, pp. 49-80.

Schaffner JH, 1909. The classification of plants, IV. Ohio Naturalist 9: $446-455$.
Schmitt I, Mueller G, Lumbsch HT, 2005. Ascoma morphology is homoplaseous and phylogenetically misleading in some pyrenocarpous lichens. Mycologia 97: 362-374.

Schoch CL, Shoemaker RA, Seifert KA, Hambleton S, Spatafora JW, Crous PW, 2007 ['2006']. A multigene phylogeny of the Dothideomycetes using four nuclear loci. Mycologia 98: 1042-1053.

Schröter J, 1889. Pilze. In: Cohn F (ed), Kryptogamen-Flora von Schlesien, Vol. 3. J. U. Kern's Verlag, Breslau, pp. 382-386.

Schröter J, 1892. Phycomycetes. In: Engler A, Prantl K (eds), Die natürlichen Pflanzenfamilien, Vol. 1. Wilhelm Engelmann Verlag, Leipzig, pp. 63-87.

Schröter J, 1893. Monoblepharidineae. In: Engler A, Prantl K (eds), Die natürlichen Pflanzenfamilien, Vol. 1. Wilhelm Engelmann Verlag, Leipzig, pp. 106-107.

Schröter J, 1894. Pezizineae. In: Engler A, Prantl K (eds), Die natürlichen Pflanzenfamilien, Vol. 1. Wilhelm Engelmann Verlag, Leipzig, pp. 173-243.

Schüßler A, Schwarzott D, Walker C, 2001. A new fungal phylum, the Glomeromycota: phylogeny and evolution. Mycological Research 105: 1413-1421.

Seif E, Leigh L, Roewer I, Forget L, Lang BF, 2005. Comparative mitochondrial genomics in zygomycetes: bacteria-like RNase P RNAs, mobile elements and a close source of the group I intron invasion in angiosperms. Nucleic Acids Research 33: 734744.

Serbinow JL, 1907. Organisazija I rasitie nekotorich gribow Chytridineae Schröt. Scripa Botanica Horti Universitatis Imperialis Petropolitana 24: 1-147.

Shenoy BD, Jeewon R, Wu WP, Bhat DJ, Hyde KD, 2006. Ribosomal and RPB2 DNA sequence analyses suggest that Sporidesmium and morphologically similar genera are polyphyletic. Mycological Research 110: 916-928.

Sparrow FK, 1943. Aquatic Phycomycetes exclusive of the Saprolegniaceae and Pythium. University of Michigan Press, Ann Arbor.

Sparrow FK, 1958. Interrelationships and phylogeny of the aquatic Phycomycetes. Mycologia 50: 797-813.

Spatafora JW, Johnson D, Sung G-H, Hosaka K, O'Rourke B, Serdani M, Spotts R, Lutzoni F, Hofstetter V, Fraker E, Gueidan C, Miądlikowska J, Reeb V, Lumbsch T, Lücking R, Schmitt I, Aptroot A, Roux C, Miller A, Geiser D, Hafellner J, Hestmark G, Arnold AE, Büdel B, Rauhut A, Hewitt D, Untereiner W, Cole MS, Scheidegger C, Schultz M, Sipman H, Schoch CL, 2007 ['2006']. A five-gene phylogenetic analysis of the Pezizomycotina. Mycologia 98: 1020-1030.

Sprague V, Becnel JJ, 1998. Note on the name-author-date combination for the taxon Microsporidies Balbiani, 1882, when ranked as a phylum. Journal of Invertebrate Pathology 71: 91-94.

Starbäck K, 1899. Ascomyceten der Ersten Regnellschen Expedition. Bihang till Kungliga Svenska Vetenskaps-Akademiens Handlingar, ser. 3 25: 1-68.

Steenkamp ET, Wright J, Baldauf SL, 2006. The protistan origins of animals and fungi. Molecular Biology and Evolution 23: 93-106.

Sugiyama J, Hosaka K, Suh S-O, 2007 ['2006']. Early diverging Ascomycota: phylogenetic divergence and related evolutionary enigmas. Mycologia 98: 998-1007.

Suh S-O, Blackwell M, Kurtzman CP, Lachance M-A, 2007 ['2006']. Phylogenetics of Saccharomycetales, the ascomycete yeasts. Mycologia 98: 1008-1019.

Swann EC, Frieders EM, McLaughlin DJ, 2001. Urediniomycetes. In: McLaughlin DJ, McLaughlin EJ, Lemke P (eds), The Mycota. Vol. VII. Part B, Systematics and Evolution. Springer-Verlag, Berlin, pp. 37-56.

Swann EC, Taylor JW, 1995. Phylogenetic perspectives on basidiomycete systematics: evidence from the 18S rRNA gene. Canadian Journal of Botany 73: S862-S868.

Takamatsu S, 2004. Phylogeny and evolution of the powdery mildew fungi (Erysiphales, Ascomycota) inferred from nuclear ribosomal DNA sequences. Mycoscience 45: 147-157. 
Tanabe Y, O’Donnell K, Saikawa M, Sugiyama J, 2000. Molecular phylogeny of parasitic Zygomycota (Dimargaritales, Zoopagales) based on nuclear small subunit ribosomal DNA sequences. Molecular Phylogenetics and Evolution 16: 253-262.

Tanabe Y, Saikawa M, Watanabe MM, Sugiyama J, 2004. Molecular phylogeny of Zygomycota based on EF-1 and RPB1 sequences: limitations and utility of alternative markers to rDNA. Molecular Phylogenetics and Evolution 30: 438-449.

Tanabe Y, Watanabe MM, Sugiyama J, 2005. Evolutionary relationships among basal fungi (Chytridiomycota and Zygomycota): Insights from molecular phylogenetics. Journal of General and Applied Microbiology 51: 267-276.

Tang AMC, Jeewon R, Hyde KD, 2007. Phylogenetic utility of protein (RPB2, B-tubulin) and ribosomal (LSU, SSU) gene sequences in the systematics of Sordariomycetes (Ascomycota, Fungi). Antonie van Leeuwenhoek in press.

Taylor JW, Spatafora J, O'Donnell K, Lutzoni F, James T, Hibbett DS, Geiser D, Bruns TD, Blackwell M, 2004. The fungi. In: Cracraft J, Donoghue MJ (eds), Assembling the Tree of Life. Oxford University Press, Oxford, pp. 171-196.

Tehler A, 1988. A cladistic outline of the Eumycota. Cladistics 4: 227-277.

Tehler A, Little DP, Farris JS, 2003. The full-length phylogenetic tree from 1551 ribosomal sequences of chitinous fungi, Fungi. Mycological Research 107: 901-916.

Thorn RG, Moncalvo J-M, Reddy CA, Vilgalys R, 2000. Phylogenetic analyses and the distribution of nematophagy support a monophyletic Pleurotaceae within the polyphyletic pleurotoid-lentinoid fungi. Mycologia 92: 241-252.

Tibell L, Vinuesa M, 2005. Chaenothecopsis in a molecular phylogeny based on nuclear rDNA ITS and LSU sequences. Taxon 54: 427-442.

Tibell L, Wedin M, 2000. Mycocaliciales, a new order for nonlichenized calicioid fungi. Mycologia 92: 577-581.

Underwood LM, 1899. Moulds, mildews and mushrooms: a guide to the systematic study of the Fungi and Mycetozoa and their literature. Henry Holt, New York.

Vijaykrishna D, Mostert L, Jeewon R, Gams W, Hyde KD, Crous PW, 2004. Pleurostomophora, an anamorph of Pleurostoma (Calosphaeriales), a new anamorph genus morphologically similar to Phialophora. Studies in Mycology 50: 387-395.

Vossbrinck CR, Debrunner-Vossbrinck BA, 2005. Molecular phylogeny of the Microsporidia: ecological, ultrastructural and taxonomic considerations. Folia Parasitologica 52: 131-142.

Wagner T, Fischer M, 2002. Proceedings towards a natural classification of the worldwide taxa Phellinus s.l. and Inonotus s.l., and phylogenetic relationships of allied genera. Mycologia 94: 998-1016.

Walker C, Schüßler A, 2004. Nomenclatural clarifications and new taxa in the Glomeromycota. Mycological Research 108: 981-982.

Wang Z, Binder M, Schoch C, Johnston PR, Spatafora JW, Hibbett DS, 2006. Evolution of helotialean fungi (Leotiomycetes, Pezizomycotina): a nuclear rDNA phylogeny. Molecular Phylogenetics and Evolution 41: 295-312.

Wang Z, Johnston PR, Takamatsu S, Spatafora JW, Hibbett DS, 2007 ['2006']. Phylogenetic classification of the Leotiomycetes based on rDNA data. Mycologia 98: 1066-1076.
Wedin M, Wiklund E, Crewe A, Döring H, Ekman S, Nyberg A Schmitt I, Lumbsch HT, 2005. Phylogenetic relationships of Lecanoromycetes (Ascomycota) as revealed by analyses of mtSSU and nLSU rDNA sequence data. Mycological Research 109: 159172.

Weir A, Blackwell M, 2001. Molecular data support the Laboulbeniales as a separate class of Ascomycota, Laboulbeniomycetes. Mycological Research 105: 1182-1190.

Weiß M, Bauer R, Begerow D, 2004a. Spotlights on heterobasidiomycetes. In: Agerer R, Piepenbring M, Blanz P (eds), Frontiers in Basidiomycote Mycology. IHW Verlag, Eching, pp. 7-48.

Weiß M, Oberwinkler F, 2001. Phylogenetic relationships in Auriculariales and related groups-hypotheses derived from nuclear ribosomal DNA sequences. Mycological Research 105 403-415.

Weiß M, Selosse M-A, Rexer K-H, Urban A, Oberwinkler O, 2004b. Sebacinales: a hitherto overlooked cosm of heterobasidiomycetes with a broad mycorrhizal potential. Mycological Research 108: 1003-1010.

Wells K, Bandoni RJ, 2001. Heterobasidiomycetes. In: McLaughlin DJ, McLaughlin EJ, Lemke P (eds), The Mycota. Vol. VII. Part B, Systematics and Evolution. Springer-Verlag, Berlin, pp. 85-120.

White TJ, Bruns TD, Lee S, Taylor JW, 1990. Amplification and direct sequencing of fungal ribosomal RNA genes for phylogenetics. In: Innis MA, Gelfand DH, Sninsky J, White TJ (eds), PCR Protocols: a Guide to Methods and Applications. Academic Press, San Diego, pp. 315-322.

White MM, James TY, O'Donnell K, Cafaro MJ, Tanabe Y, Sugiyama J, 2007 ['2006']. Phylogeny of the Zygomycota based on nuclear ribosomal sequence data. Mycologia 98: 872-884.

Whittaker RH, 1959. On the broad classification of organisms. Quarterly Review of Biology 34: 210-226.

Wiklund E, Wedin M, 2003. The phylogenetic relationships of the cyanobacterial lichens in the Lecanorales suborder Peltigerineae. Cladistics 19: 419-431.

Wingfield B, Viljoen CD, Wingfield MJ, 1999. Phylogenetic relationships of ophiostomatoid fungi associated with Protea infrusctescences in South Africa. Mycological Research 103: 16161620.

Winka K, 2000. Phylogenetic relationships within the Ascomycota based on $18 \mathrm{~S}$ rDNA sequences. PhD thesis, Umeå University, Umeå.

Winter G, 1880. Schizomyceten, Saccharomyceten und Basidiomyceten. In: Rabenhorst's Kryptogamen-Flora von Deutschland, Oesterreich und der Schweiz, Vol. 1. E. Kummer, Leipzig, pp. 1-924.

Woronichin NN, 1925. Über die Capnodiales. Annales Mycologici 23: 174-178.

Zalar P, de Hoog GS, Schroers H-J, Frank JM, Gunde-Cimerman N, 2005. Taxonomy and phylogeny of the xerophilic genus Wallemia (Wallemiomycetes and Wallemiales, cl. et ord. nov.). Antonie van Leeuwenhoek 87: 311-328.

Zhang N, Castlebury LA, Miller AN, Huhndorf S, Schoch CL, Seifert K, Rossman AY, Rogers JD, Kohlmeyer J, VolkmannKohlmeyer B, Sung G-H, 2007 ['2006']. Sordariomycetes systematics: an overview of the systematics of the Sordariomycetes based on a four-gene phylogeny. Mycologia 98: 1077-1088. 